\title{
Mechanism of the Bauschinger Effect in Al-Ge-Si Alloys
}

\author{
Wei Gan ${ }^{\mathrm{a}}$, Hyuk Jong Bong ${ }^{\mathrm{b}}$, Hojun Lim ${ }^{\mathrm{c}}$, R.K. Boger ${ }^{\mathrm{d}}$, F. Barlat ${ }^{\mathrm{e}}$, and R. H. Wagoner ${ }^{\mathrm{b}, 1}$ \\ ${ }^{a}$ Medtronic, Mounds View, MN 55112, USA \\ ${ }^{\mathrm{b}}$ The Ohio State University, Dept. Materials Science and Engineering, Columbus, OH 43210, USA \\ ${ }^{c}$ Sandia National Laboratories, Dept. Comp. Materials and Data Science, Albuquerque, NM 87123, USA \\ ${ }^{\mathrm{d}}$ Dassault Systemes, Mason, OH 45040, USA \\ ${ }^{\mathrm{e}}$ Pohang University of Science and Technology, GIFT, Pohang, Gyeongbuk, 37673, Rep. of Korea
}

\begin{abstract}
Wrought $\mathrm{Al}-\mathrm{Ge}-\mathrm{Si}$ alloys were designed and produced to ensure dislocation bypass strengthening ("hard pin" precipitates) without significant precipitate cutting/shearing ("soft pin" precipitates). These unusual alloys were processed from the melt, solution heat treated and aged. Aging curves at temperatures of $120,160,200$ and $240^{\circ} \mathrm{C}$ were established and the corresponding precipitate spacings, sizes, and morphologies were measured using TEM. The role of non-shearable precipitates in determining the magnitude of Bauschinger was revealed using large-strain compression/tension tests. The effect of precipitates on the Bauschinger response was stronger than that of grain boundaries, even for these dilute alloys. The Bauschinger effect increases dramatically from the under-aged to the peak aged condition and remains constant or decreases slowly through over-aging. This is consistent with reported behavior for $\mathrm{Al}-\mathrm{Cu}$ alloys (maximum effect at peak aging) and for other $\mathrm{Al}$ alloys (increasing through over-aging) such as $\mathrm{Al}-\mathrm{Cu}-\mathrm{Li}, \mathrm{Al}$ 6111, Al 2524, and Al 6013. The Al-Ge-Si alloy response was simulated with three microstructural models, including a novel SD (SuperDislocation) model, to reveal the origins of the Bauschinger effect in dilute precipitation-hardened / bypass alloys. The dominant mechanism is related to the elastic interaction of polarized dislocation arrays (generalized pile-up or bow-out model) at precipitate obstacles. Such effects are ignored in continuum and crystal plasticity models.
\end{abstract}

\section{Keyword}

Al-Ge-Si; Bauschinger effect; precipitate; Orowan bypass; Super-Dislocation model

Manuscript submitted to Materials Science and Engineering A, October 21, 2016. Manuscript revised, December 03, 2016.

${ }^{1}$ Corresponding Author. Tel.: +1 614292 2079; E-mail address: wagoner.2@ osu.edu 


\section{INTRODUCTION}

Many commercial and model aluminum alloys utilize fine precipitates for strengthening by serving as obstacles to dislocation movement. Such hardening, among other types, can give rise to a transient hardening response upon reverse or non-proportional loading. This transient response is general is referred to as the "Bauschinger effect" (with "effect" uncapitalized) [1], although this term is often used in the more limited sense of simply a reduced yield stress upon a stress reversal (which will be referred to as the "Bauschinger Effect," capitalized).

An accurate description of precipitation strengthening and its role in reverse yielding and hardening behavior have importance for, among other things, simulation of sheet forming operations, where stress reversals and non-proportional strain paths are common. Springback is particularly sensitive to the stress under such conditions [2-10]. The generalized Bauschinger effect represents a significant departure from proportional-path stresses for strains typically up to a few \% strain following the reversal [11-15]. At the smallest strains following reversal, there is pronounced nonlinearity that signals the start of such transient behavior [16-18]. The nonlinearity before nominal yield was recently shown to be significant for other than springback applications, in particularly the crush of automotive structural components [16].

The mechanisms that give rise to the transient response fall into two categories: continuum $^{2}$ and dislocation-based models. Continuum models capture stress/strain inhomogeneities but have no intrinsic length scale, such as a Burgers vector, and therefore the scale of the microstructure is irrelevant. That is, coarsening of precipitates of fixed volume fraction would not affect the magnitude of the Bauschinger effect. Examples include phenomenological kinematic hardening models [19,20] following the linear kinematic hardening methods proposed by Prager [21] and Ziegler [22]. Such models fail to capture the Bauschinger effect observed in pure single crystal materials where no second phase or grain texture effect is present $[23,24]$.

The strength of the Bauschinger effect within the continuum approach depends directly on the volume fraction of second-phase particles. Thus, only composites [25], or smaller-scale alloys with large volume fractions such as dispersion-hardened aluminum [26,27] or dual-phase steels [28] are likely to have Bauschinger behavior dominated by continuum-scale elasto-plasticity ${ }^{3}$. For age-hardened aluminum alloys where

${ }^{2}$ Crystal plasticity models that are constructed grain-by-grain (CP-FEM) are a variant of continuum models in that each grain is treated as a continuum although grain texture (another source of inhomogeneity) is taken into account. The important distinction for the current work is that neither CP not continuum treatments take into account the elastic interaction of dislocations and the inherent length scale of the Burgers vector therein.

${ }^{3}$ In fact, recent work by the Wagoner group [120] shows that even in the case of DP 980 steel (UTS greater than $980 \mathrm{MPa}$ ), with large martensite islands of $32 \%$ volume fraction, the continuum models capture only a small fraction of the observed Bauschinger effect. 
second-phase particles represent less than 3\% volume fraction, finite element (FE) simulations show only a minimal Bauschinger effect, disappearing after strains of the order of the elastic strain following a stress reversal (see the Simulations and Discussion section of this paper).

A basic single-dislocation mechanism for transient yielding and hardening following a stress reversal was proposed by Orowan, who showed that the dislocation looping mechanism can produce significant Bauschinger effects for small volume fractions of closely-spaced particles [29-31]. Elastic and plastic inclusion theories quantify the amount of strengthening and Bauschinger effect from precipitates [32,33].

Multi-dislocation models conceptually involve the storage and release of elastically-interacting polarized sets of dislocations during stress reversals, producing a transient behavior over a finite strain range (corresponding to the strain needed to propel the dislocations to a new steady-state configuration in the new strain path). These sets can be thought of as generalized dislocation pile-ups or bow-outs [34-37] corresponding to simple models of small numbers of individual dislocation. TEM observations have revealed complex, and difficult to analyze, dislocation interactions during such stress reversal or path changes [38-47].

Semi-phenomenological models based on dislocation cell structures and slip band evolution [42,48-55] are difficult to verify. Some experimental results show that details of the dislocation cell structure do not have a determinant role in the Bauschinger effect [56].

At the macroscopic level, the Bauschinger effect in binary $\mathrm{Al}-\mathrm{Cu}$ alloys was originally reported to be strongest at peak aging (i.e. maximum strength) [57,58], consistent with a looping /storage mechanism and a presumption of non-shearable precipitates. However, the original Al-Cu results from the 1980's are not consistent with more recent results for Al-Cu-Sn [19] and Al-Cu-Li alloys [59,60], which exhibit a Bauschinger effect that continues to increase after peak aging, or at least remains nearly constant or decreases very slowly. Complex commercial alloys 6111 [61], 2524 and 6013 [62,63] commercial $\mathrm{Al}$ alloys with an array of particles types, sizes, spacings and coherency exhibit a Bauschinger effect that continues to grow well into the overaging conditions (i.e. for a macroscopically weaker condition featuring large, widely spaced particles) [62].

In all of these commercial alloy systems, the mechanistic interaction of dislocations with precipitates is complicated by a transition from shear-able, small, coherent, metastable particles at early aging stages, to large, non-shearable, stable, incoherent, particles in late aging stages [20,53,54,57-59,61,64]. (In the Orowan mechanism vernacular, from "soft pins" to "hard pins ".") In contrast, age-hardened Al-Ge-Si alloys are known to have finely spaced Ge-Si precipitates of stable [65,66], incoherent [65], non-shearable

\footnotetext{
4 "Hard pins" are obstacles to dislocation motion that must be bypassed (looped around) during plastic deformation, as opposed to "soft pins" which may be cut or sheared without significant looping around them by the impinging dislocations.
} 
[65,67], fixed-composition precipitates with diamond-cubic crystal structures [65,67] at nearly all sizes [65]. Under these conditions, only dislocation bypass is expected, no shearing is possible except for vanishingly small particles. The critical size is estimated below, and the bypass-only nature of the actual Ge-Si precipitates in the current alloys is verified later in this paper.

The critical size of incoherent particles for the transition from shearing to bypassing can be also estimated by using the following equation $[65,68]$ :

$$
d_{c}=\frac{4 \pi b \mu_{M}}{\mu_{P}}
$$

where $\mu_{\mathrm{M}}$ and $\mu_{\mathrm{P}}$ are the shear moduli of the Al matrix and the Ge/Si particle, respectively. Using the shear moduli data (Al:26 GPa, Si:51-81 GPa and Ge:41 GPa). Equation 1 provides the following estimates of the critical size of incoherent particles in $\mathrm{Al}: \quad 1.2 \mathrm{~nm}$ $(100 \% \mathrm{Si}), 2.3 \mathrm{~nm}(100 \% \mathrm{Ge})$. As will be shown below, the precipitates of sizes down to these limits were resolvable in the aged Al-Ge-Si alloys used here, and none were observed having sizes smaller than $6 \mathrm{~nm}$. Therefore, for all practical purposes, aged Al-Ge-Si alloys contain only non-shearable precipitates.

The Al-Ge-Si system therefore offers an opportunity to observe the transient behavior at all aging stages without the confounding complexity of changing mechanism between shearing and bypass. This allows the characteristics of "hard pin" strengthening to be revealed experimentally.

Three Al-Ge-Si systems with various alloy concentrations were produced. The materials were aged for various times at various temperatures, and their precipitate structure (shape, size and spacing) were measured by transmission electron microscope (TEM). Tensile and compression/tension (CT) tests were conducted to obtain the monotonic and reverse hardening behavior of the aged materials. FE simulations were compared with experiments to shed light on the nature of the micro-mechanism producing the Bauschinger effect in "hard pin" strengthened alloys.

Note: While the primary purpose of the current work is to reveal the nature of the mechanism(s) producing the Bauschinger effect by using specially-prepared alloys, some effort was also expended to measure and report systematically the aging behavior of Al-Ge-Si because such basic metallurgical data is rare for this system.

\section{EXPERIMENTAL AND ANALYTICAL PROCEDURES}

Dilute Al-Ge-Si alloys were processed at the Alcoa Technical Center [69]. Three melt chemistries were cast into ingots with nominal dimensions of $51 \mathrm{~mm} \times 254 \mathrm{~mm} \times 356 \mathrm{~mm}$, 
homogenized for $8 \mathrm{~h}$ at $\left(500^{\circ} \mathrm{C}\right.$, and forced-air cooled. Hot rolling at $440^{\circ} \mathrm{C}$ was carried out using 12 passes reducing the initial thickness of $51 \mathrm{~mm}$ to a final thickness of $5 \mathrm{~mm}$. Cold rolling reduced the strip to its final thickness of $2 \mathrm{~mm}$ in 3 passes.

\subsection{Compositions}

Table 1 represents the compositions of the three alloys. For reasons described later, only the richest alloy was characterized extensively. For this alloy, Table 2 compares theoretical precipitate volume fraction (i.e. assuming no solubility of $\mathrm{Si}$ and $\mathrm{Ge}$ in $\mathrm{Al}$ at room temperature, which is in fact less than 0.01\% [70-72]) with the TEM-measured volume fractions. Less than $20 \%$ of the $\mathrm{Ge}$ and $\mathrm{Si}$ has been precipitated in the under-aged condition (as defined later) whereas all of it (within experimental uncertainty) has been precipitated by the peak-aged condition.

Table 1. Compositions of three Al-Ge-Si alloys (atomic \%)

\begin{tabular}{|l|c|c|c|c|c|c|c|}
\hline & $\mathrm{Al}$ & $\mathrm{Ge}$ & $\mathrm{Si}$ & $\mathrm{Fe}$ & $\mathrm{Cu}$ & $\mathrm{Zn}$ & $\mathrm{Ti}$ \\
\hline $\mathrm{Al}-1 \% \mathrm{Ge}-\mathrm{Si}$ & Balance & 0.49 & 0.50 & 0.03 & 0.01 & 0.03 & 0.02 \\
\hline $\mathrm{Al}-0.4 \% \mathrm{Ge}-\mathrm{Si}$ & Balance & 0.19 & 0.16 & 0.03 & 0.00 & 0.01 & 0.01 \\
\hline $\mathrm{Al}-0.2 \% \mathrm{Ge}-\mathrm{Si}$ & Balance & 0.07 & 0.16 & 0.02 & 0.00 & 0.01 & 0.01 \\
\hline
\end{tabular}

Table 2. Estimated volume percentages of the Ge/Si precipitates, assuming no residual solutes

\begin{tabular}{|l|c|c|c|c|}
\hline & Theoretical & \multicolumn{3}{|c|}{ Measured } \\
\cline { 2 - 5 } & & UA & PA & OA \\
\hline $\mathrm{Al}-1 \% \mathrm{Ge}-\mathrm{Si}$ & $1.25 \%$ & $0.2 \%$ & $1.2 \%$ & $1.2 \%$ \\
\hline $\mathrm{Al}-0.4 \% \mathrm{Ge}-\mathrm{Si}$ & $0.46 \%$ & - & - & - \\
\hline $\mathrm{Al}-0.2 \% \mathrm{Ge}-\mathrm{Si}$ & $0.29 \%$ & - & - & - \\
\hline
\end{tabular}

\subsection{Aging and Precipitate Characterization}

Test coupons $30 \mathrm{~mm} \times 30 \mathrm{~mm}$ were sheared from the rolled sheets, solution treated in air at $500^{\circ} \mathrm{C}$ for $1 \mathrm{~h}$ then quenched in water. Aging was conducted at 120, 160, 200 and $240^{\circ} \mathrm{C}$ using a Scientific, Isotemp ${ }^{\circledR}$ Model $725 \mathrm{~F}$ furnace. A redundant aluminum sheet was preheated in the oven and the samples were placed on top of it in order to increase the temperature rise rate in test coupons for more precise determination of aging times. Micro-hardness data were collected at all aging times and temperatures.

The microstructures of the as-received and heat-treated samples were examined using a Philips CM200 transmission electron microscope at $200 \mathrm{kV}$. The TEM samples were mechanically thinned $\sim 120 \mu \mathrm{m}$, and electro-polished in $70 \mathrm{vol} \%$ methanol and $30 \mathrm{vol} \%$ nitric acid to $150 \mathrm{~nm}$ at the center of the sample. A twin jet polisher was used with a at $-30{ }^{\circ} \mathrm{C}$ and a potential of $15 \mathrm{~V}$. 
Precipitate size and spacing were collected from TEM images. The maximum magnification was 350,000 times, allowing resolution of precipitates with diameters $(d)$ down to $1 \mathrm{~nm}$. Few precipitates were observed with sizes less than $5 \mathrm{~nm}$. Three to five views were used and approximately 300 particles were measured manually for each aging condition to obtain spacing $(\lambda)$ and volume fraction $(f)$.

The average precipitate size, $d$, for the aging condition represents the arithmetic mean of all the measured individual particle sizes $\left(d_{p p t}\right)$, where for non-equiaxed precipitates the size is obtained from measured minimum and maximum linear dimensions $\left(d_{\min }\right.$ and $\left.d_{\min }\right)$ using $d_{p p t}=\sqrt{d_{\min } d_{\max }}$ ). Scatter is $3 \sim 5 \mathrm{~nm}$, depending on aging condition (details in Section III.A). The total volume fraction of the precipitates was calculated using the following Equation 2 [72]:

$$
f=\frac{\pi d^{3} N}{6 A(t+d)}
$$

(Equation 2)

where $A$ is the area of the micrograph and $N$ is the total number of particles in that area. The foil thickness, $t$, was measured using the convergent beam electron diffraction (CBED) method $[73,74]$. Once the particle size and volume fraction were computed, the inter-particle spacing, $\lambda$, was obtained using Equation 3 [75]:

$$
\lambda=(1.23 \sqrt{2 \pi / 3 f}-2 \sqrt{2 / 3})(2 / d)
$$

(Equation 3)

where $r$ is the particle radius.

\subsection{Mechanical Testing}

Vickers micro-hardness tests were conducted with a load of $100 \mathrm{~g}$ and $20 \mathrm{~s}$ of dwell time on polished sample surfaces. Monotonic tensile tests (rolling direction) were conducted at a nominal strain rate of $1.7 \times 10^{-3} / \mathrm{s}$. using standard ASTM E- 8 tensile samples [76].

In order to allow correlation of Vickers micro-hardness (HV) with standard stress measures from tensile tests, measured values were compared for the current Al-Ge-Si alloys and for a few other aluminum alloys as shown in Figures 1 (a) - (d). The other alloys are commercial aluminum alloys ( $\mathrm{Al} 2524$ and $\mathrm{Al}$ 6013) [62,77] and two friction stir welded (FSW) alloys (Al 5083-H18 and Al 6111-T4) [78]. The correlation between tensile strength and Vickers hardness is nearly linear, while the best correlation with HV is to the "flow stress," $0.5\left(\sigma_{y}+\sigma_{U T S}\right)$ where $\sigma_{y}$ and $\sigma_{U T S}$ are the yield stress and ultimate tensile strength, respectively. 


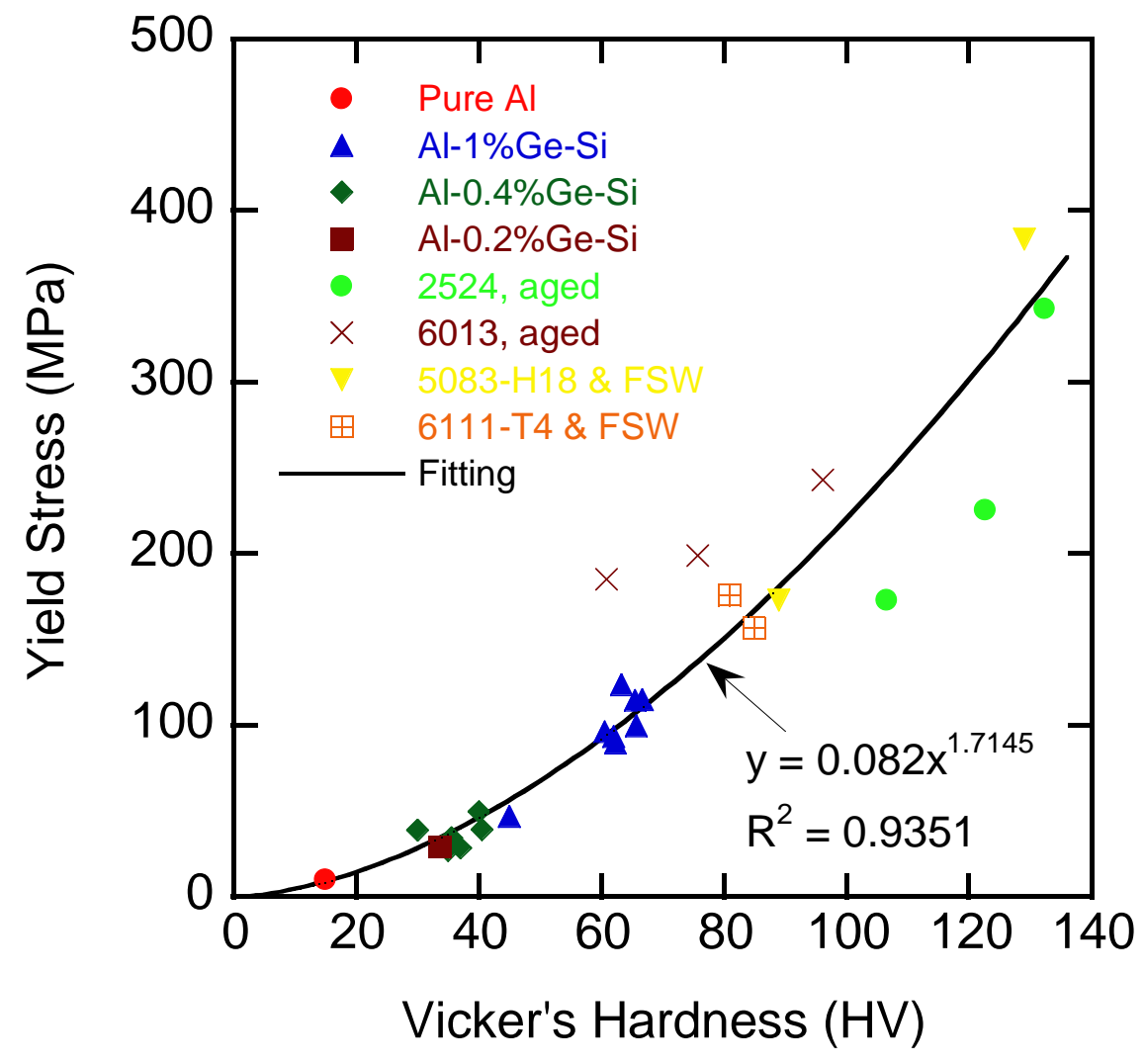

(a) 


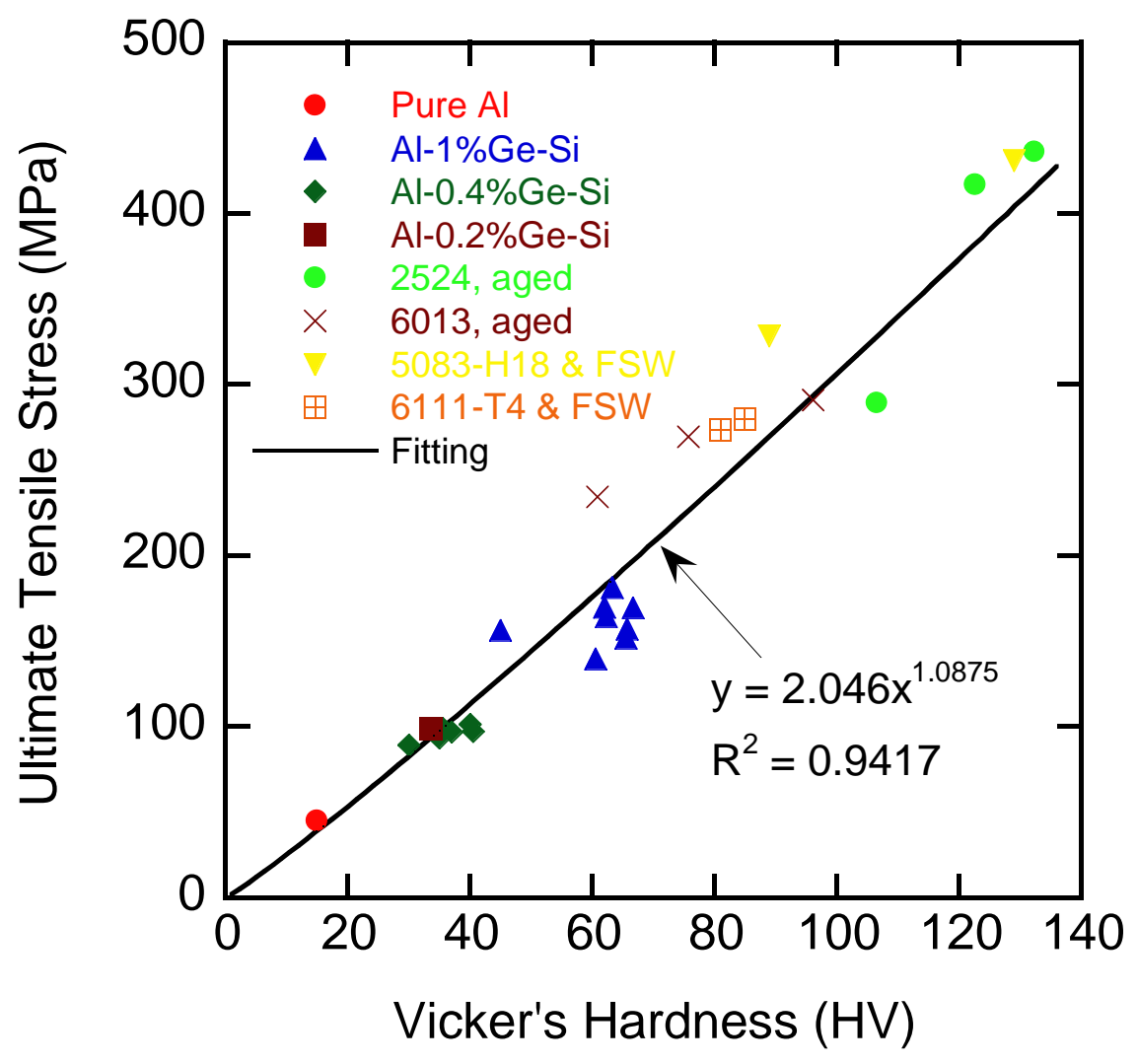

(b) 


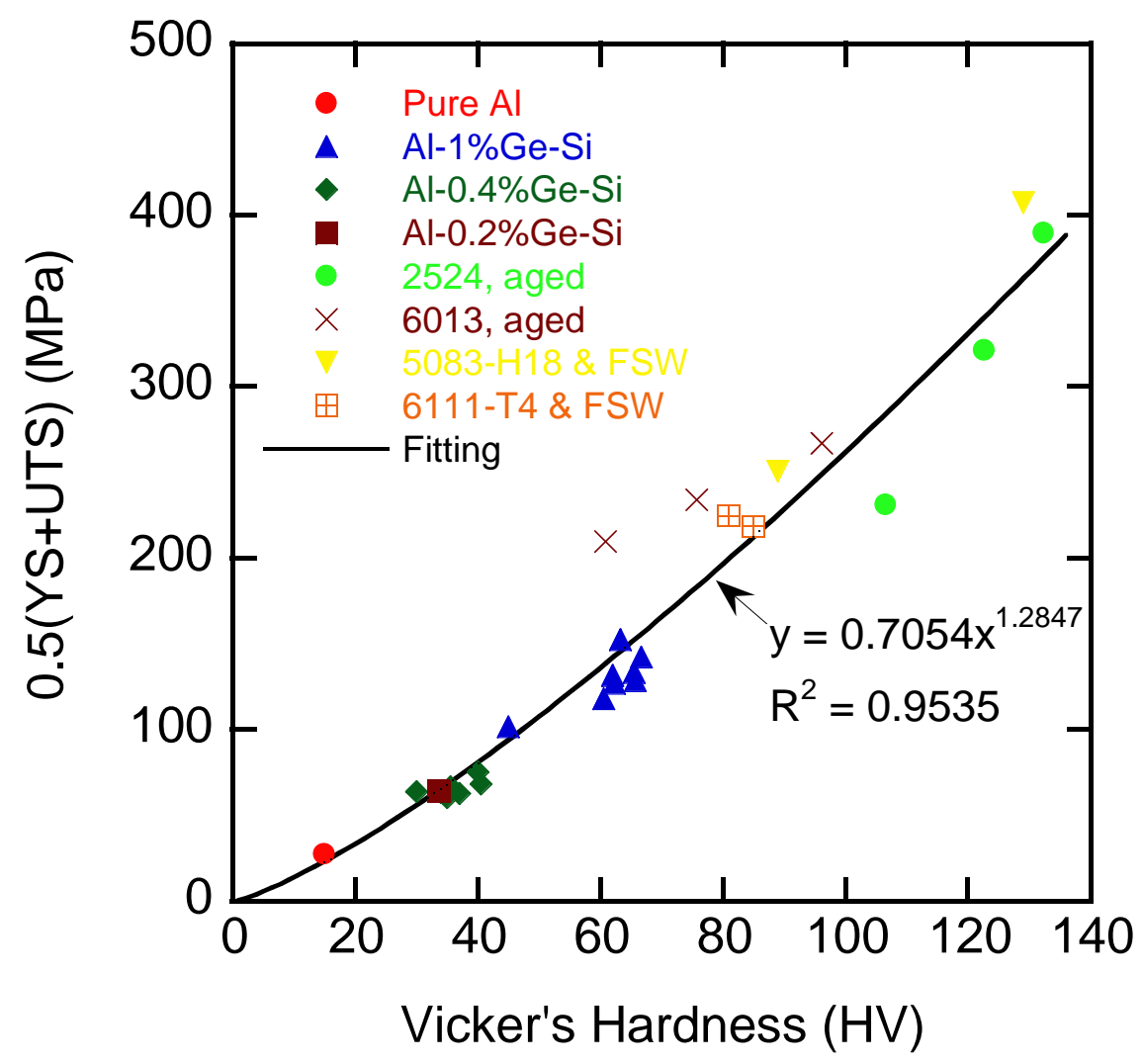

(c) 


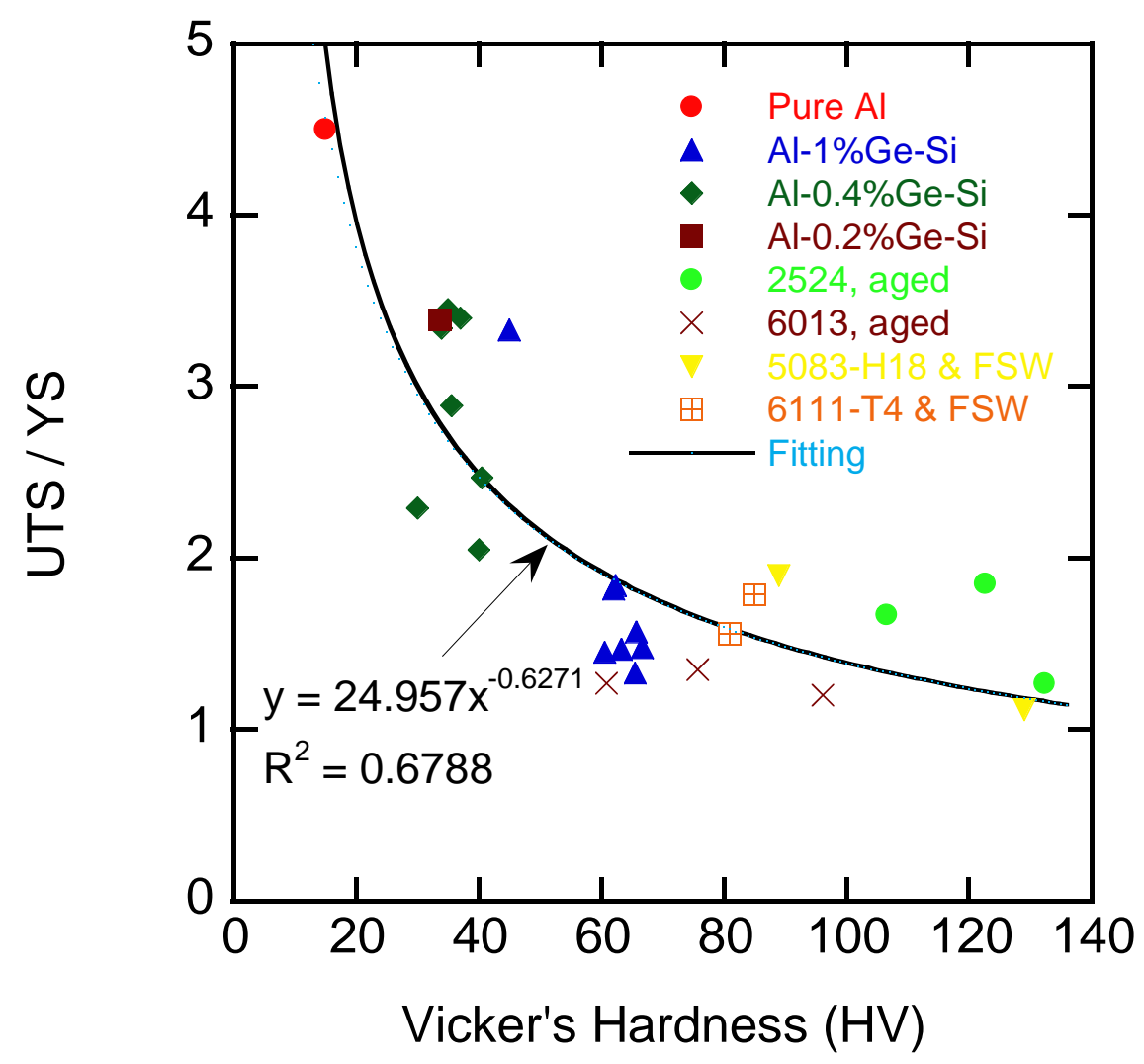

(d)

Figure 1. Tensile properties and Vickers hardness (HV) correlations for various aluminum alloys: (a) $\sigma_{y}$ vs. $\mathrm{HV}$, (b) $\sigma_{U T S}$ vs. $\mathrm{HV}$, (c) $0.5\left(\sigma_{y}+\sigma_{U T S}\right)$ vs. $\mathrm{HV}$, and (d)

$$
\sigma_{U T S} / \sigma_{y} \text { vs. HV }
$$

In addition to standard tensile tests, special compression tension (CT) tests in the rolling direction were performed to reveal the Bauschinger effect. These tests use a special sample and a special fixture that applies side forces to prevent buckling at large compressive strains [77-81]. A non-contact $\mathrm{EIR}^{\mathrm{TM}}$ laser extensometer [82] was used to measure the strain during deformation. Measured stress-strain data were corrected for biaxial stress and friction effects as presented in detail elsewhere [77]. The friction coefficient used in the correction procedure was determined by matching the friction-adjusted tensile curve with the corresponding results from standard tension tests.

\subsection{Bauschinger Effect Characterization}


The traditional measure of the Bauschinger Effect ${ }^{5}$ is the "Bauschinger Factor" [83,84], which will be called here the "Bauschinger Stress Factor" to differentiate it from other measures introduced below. $\beta$ measures the relative stress magnitude with and without a stress reversal, defined as follows:

$$
\beta=\frac{\sigma_{f}+\sigma_{r}}{\sigma_{f}}
$$

where $\sigma_{f}$ is the signed forward stress before the strain path change and $\sigma_{r}$ is the signed yield stress (however defined) after the path change. If there is no Bauschinger effect, $\sigma_{r}=-\sigma_{f}$, and $\beta=0$. When yielding occurs at zero stress upon path reversal, $\beta=1$. The value of $\beta$ depends intimately on the choice of yield criterion, with $\beta_{0.2}$ denoting a standard $0.2 \%$ offset criterion (yield stress $=\sigma_{r, 0.2}$ ) and $\beta_{0.4}$ denoting a $0.4 \%$ offset yield stress criterion (yield stress $=\sigma_{r, 0.4}$ ). The latter criterion is tested based on observations of excessive scatter of the usual yield stress for similar CT tests of $\mathrm{Mg}$ [85] and $\mathrm{Al}[62]$.

Two additional measures were introduced to extend the characterization of the post-reversal plastic ${ }^{6}$ behavior: 1) the "Bauschinger Strain Factor," $\gamma$, represents the strain extent of the disturbance of the path reversal (similar to a half-life of a transient), and 2) the "Bauschinger Energy Factor," $\Delta E$, represents the difference in work dissipated (plastic work) between the monotonic and reverse deformation after the reversal strain. The quantitative definitions of these new measures are illustrated with the aid of CT and tensile test results for an example case: Al-1\% Ge-Si, aged for $12 \mathrm{~h}$, Figure 2.

The Bauschinger Strain Factor $\gamma$ is the increment of strain following reverse yield to the point at which the absolute slope of the reverse loading curve ${ }^{7}$ is twice ${ }^{8}$ the absolute slope of the corresponding monotonic slope (i.e. the slope of a companion tensile test).

5 "Bauschinger Effect" (both words capitalized) here refers the difference of yield stress following a stress reversal as compared with the flow stress just prior to reversal.

6 In the current work, the fine distinctions between plastic and elastic behavior at small strains, including pre-yield nonlinearity [16-18] before yield are ignored. In the current work, the initial reverse loading is considered linear up to yielding, where yielding is determined conventionally by an offset criterion.

${ }^{7}$ All stresses and strains are presented as absolute values for more meaningful, and more simply interpreted, plots.

8 The factor ' 2 ' was chosen as smallest integral ratio which rendered $\gamma$ definite for all of the tests. While 1 has theoretical appeal, the slope after a path reversal often never attains that value although the difference is very small. 


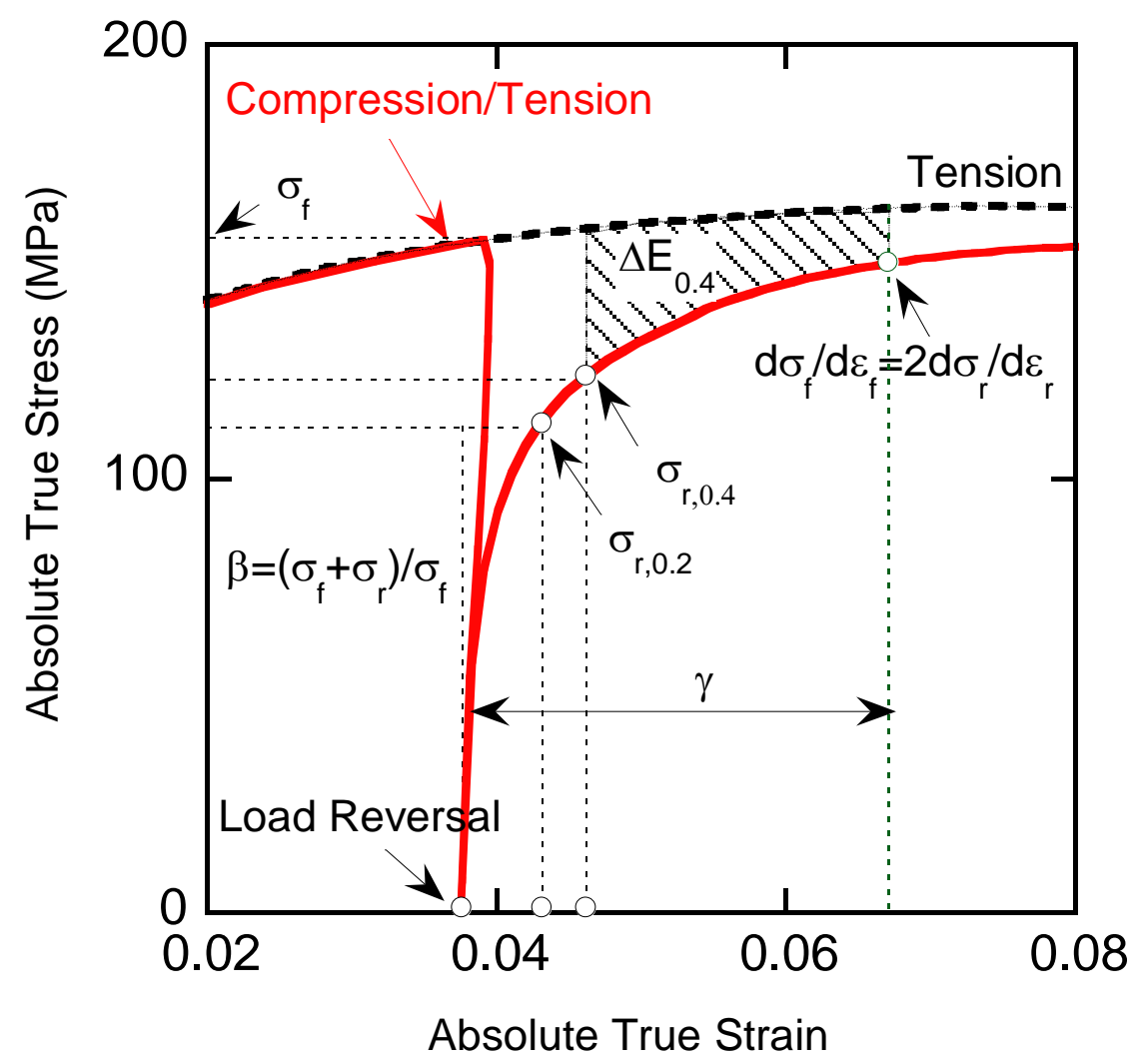

Figure 2. Schematics illustrating the definition of Bauschinger factors using CT and tensile stress-strain curves.

The Bauschinger Energy Factor $\Delta E$ is the difference in plastic work done after the reversal up to an incremental strain of 0.03 starting from 1) zero stress, 2) the $0.2 \%$ offset yield stress, or 3 ) the $0.4 \%$ offset yield stress. The corresponding energy factors are designated $\Delta E_{0}, \Delta E_{0.2}$, and $\Delta E_{0.4}$. ( $\Delta E_{0.4}$ is shown in Figure 2.)The plastic strains are computed using a Young's modulus of $70 \mathrm{GPa}$, a typical handbook value for Al. The choice of the strain limit of 0.03 past the reversal strain is somewhat arbitrary; it was chosen to capture a major part of the effect while minimizing the cumulative error over longer strain ranges for cases where the measured tensile and compressive stresses might include a spurious stress offset, whether by mis-calibration of load cells, inaccurate friction coefficients, or other such problems that can occur in reverse-path testing of metal sheets.

\section{RESULTS}

Results in two categories are presented. The aging Al-Ge-Si alloys is first quantified in terms of mechanical properties and microstructures. Second, the detailed mechanical 
responses for several aged conditions are analyzed to reveal the micromechanistic origins of the Bauschinger effect.

\subsection{Aging results}

The aging results for the two more-dilute alloys were judged of little interest in the current work for two reasons a) the hardening was minimal, on the order of $10 \%$ change, even after aging for several weeks at $200^{\circ} \mathrm{C}$, and b) the microstructures showed inhomogeneous distributions of precipitates, primarily near grain boundaries. For these reasons, complete aging curves were obtained only for the richest alloy produced, the $\mathrm{Al}-1 \% \mathrm{Ge}-\mathrm{Si}$ alloy, which is hereinafter referred to for simplicity as "Al-Ge-Si."

The aging curves for Al-Ge-Si at various temperatures are plotted in Figure 3, along with solution heat treated (SHT) material $(\mathrm{HV}=47)$. Preliminary characterization showed that only the lower aging temperatures $120^{\circ} \mathrm{C}$ and $160^{\circ} \mathrm{C}$ could not achieve peak aging within a week. Conversely, the highest aging temperature $240^{\circ} \mathrm{C}$ attained under-aging in less than 300s and produced marked elongated precipitates not amenable for simple quantitative analysis, Figure 4. For these reasons, the extensive characterization work was confined to 200 aging cases.

There was no detectable hardness change for SHT material over several months at room temperature. Therefore, natural aging was judged to be insignificant. To put that into perspective, pure aluminum has a Vickers hardness of 15 , corresponding to an ultimate tensile strength (UTS) of $42 \mathrm{MPa}$. This is less than one-third of the UTS in solution heat treated material $(\mathrm{HV}=47$ and $\mathrm{UTS}=150 \mathrm{MPa})$. The difference, $\sim 110 \mathrm{MPa}$, is attributable to solid solution strengthening: no GP zones or other precipitates were observed in SHT material, down to a resolution of $1 \mathrm{~nm}$. 


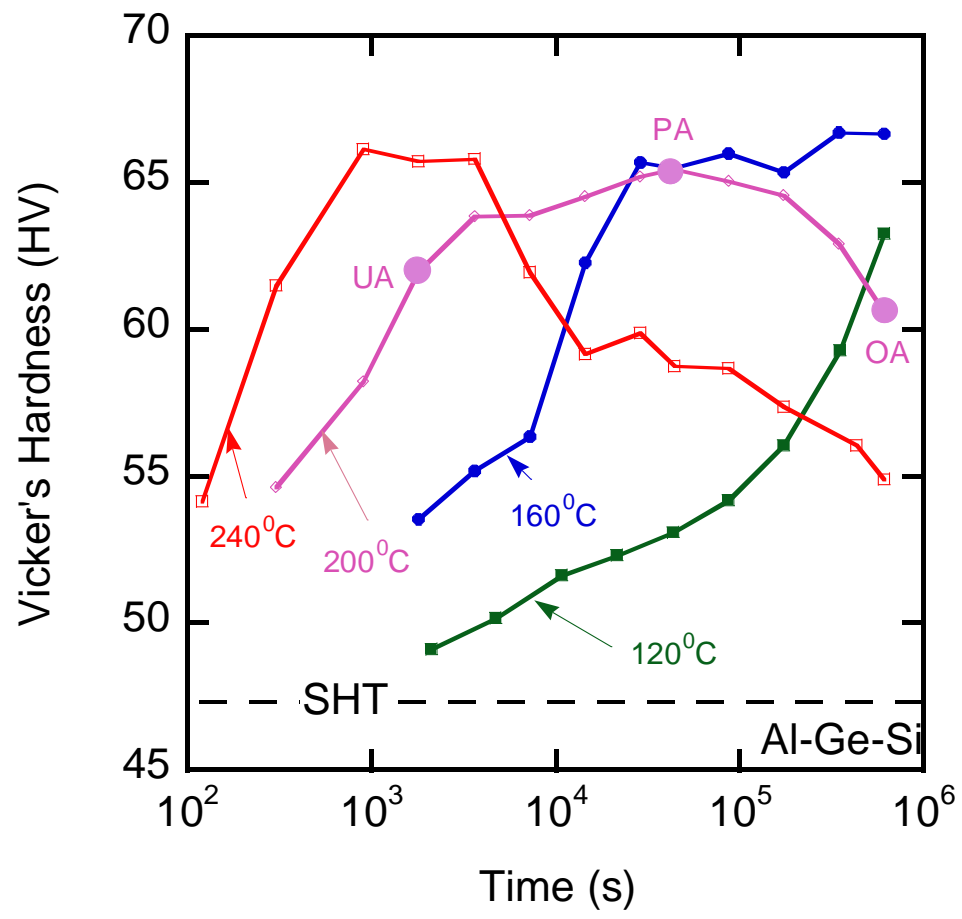

Figure 3. Aging curves of Al-Ge-Si.

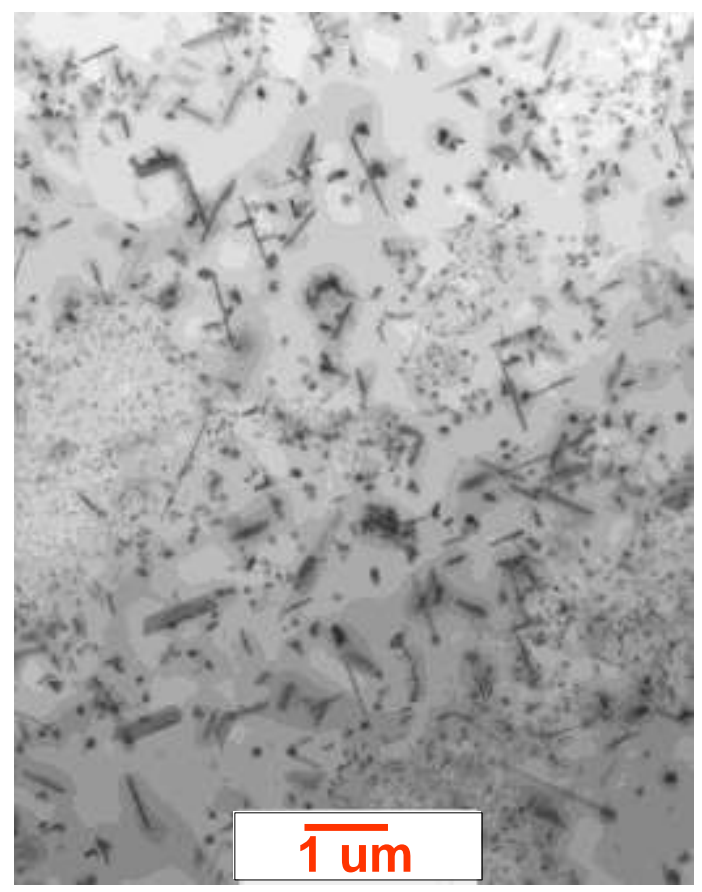

Figure 4. TEM micrograph of $\mathrm{Al}-\mathrm{Ge}-\mathrm{Si}$, aged at $240^{\circ} \mathrm{C}$ for $2 \mathrm{~h}$

\subsection{Microstructural Characterization}


TEM of the as-received Al-Ge-Si is shown in Figures 5. Figure 5(a) shows homogeneously distributed $0.5 \sim 1.0 \mu \mathrm{m}$ precipitates while Figure 5(b) shows dense dislocation networks. Solution treating $\left(500^{\circ} \mathrm{C} / 1 \mathrm{~h}\right)$ removed all visible precipitates (except for a few large inclusions near grain boundaries) and all dislocation networks, reducing the as-received hardness of $\mathrm{HV}=72$ (UTS=200 MPa) to HV=47 (UTS=150 $\mathrm{MPa})$. The very low solubility of $\mathrm{Ge}$ or $\mathrm{Si}$ in aluminum at room temperature $(<0.01 \%)$ [70-72] produces slow diffusion. Nearly all of the original solute content at solution treatment temperature is retained during rapid cooling following the solution heat treatment.

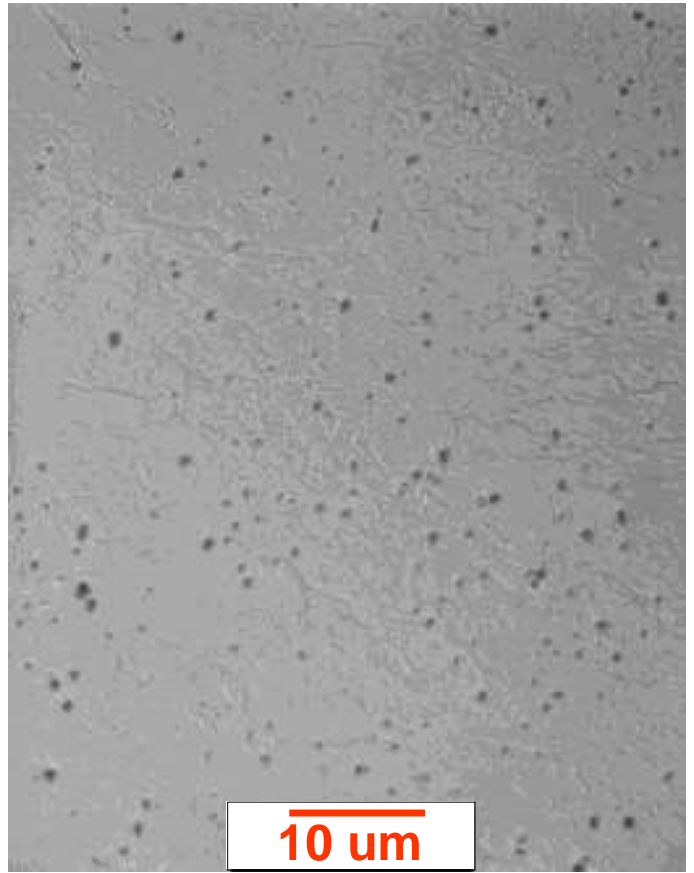

(a)

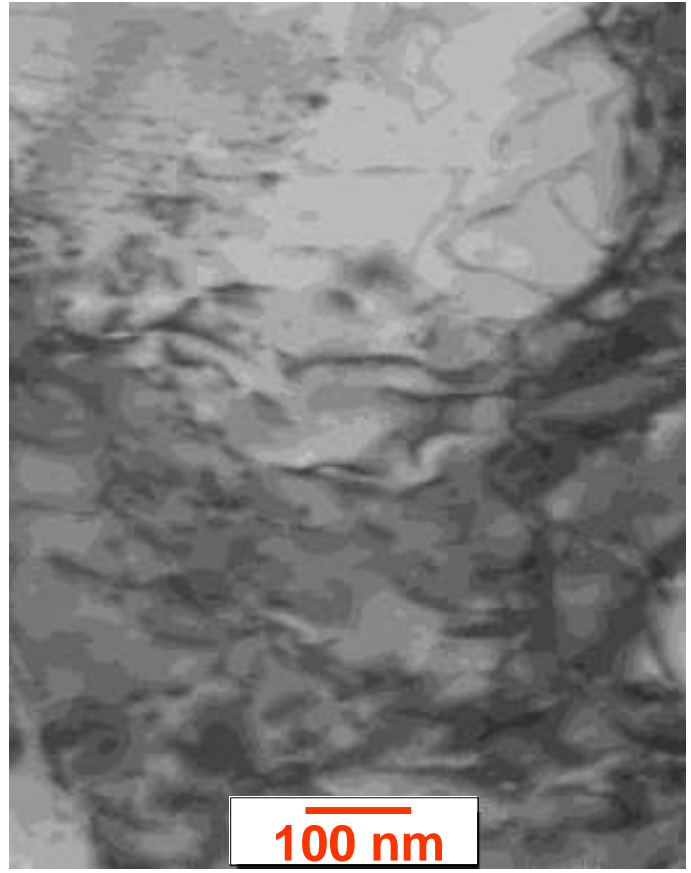

(b)

Figure 5. Microstructure of Al-Ge-Si, as-received: (a) lower magnification, and (b) higher magnification.

Contrary to the observations for the abandoned leaner alloys, evenly distributed $0.5 \sim 1.0$ $\mu \mathrm{m}$ precipitates were apparent in most of the aging conditions for Al-Ge-Si. Figures 6 show the precipitate structures of the material after aging times of $0.5 \mathrm{~h}, 12 \mathrm{~h}$ and one week at $200^{\circ} \mathrm{C}$ corresponding respectively to the UA, PA and OA conditions in Figure 3. Virtually all of the precipitates in the OA condition grew into equiaxed shape; no laths or plates were observed. The quantitative precipitate size distribution for $200^{\circ} \mathrm{C}$ aging is shown in Figure 7. 


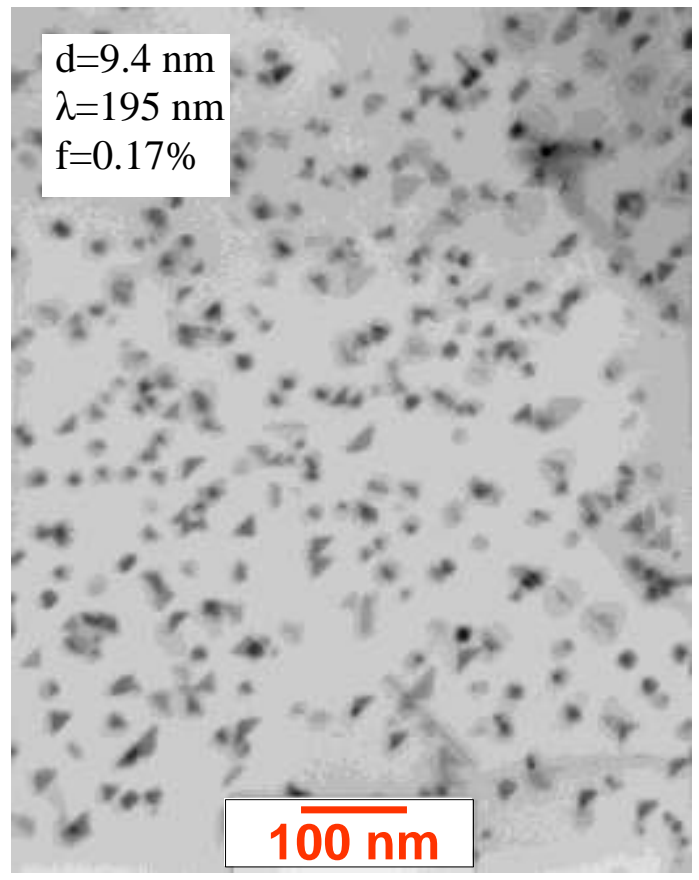

(a)

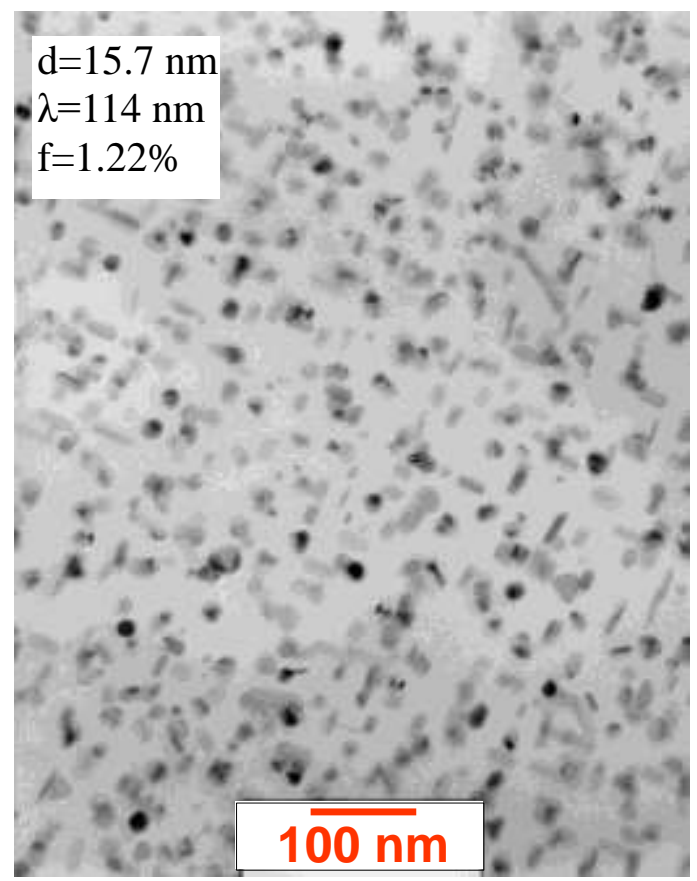

(b)

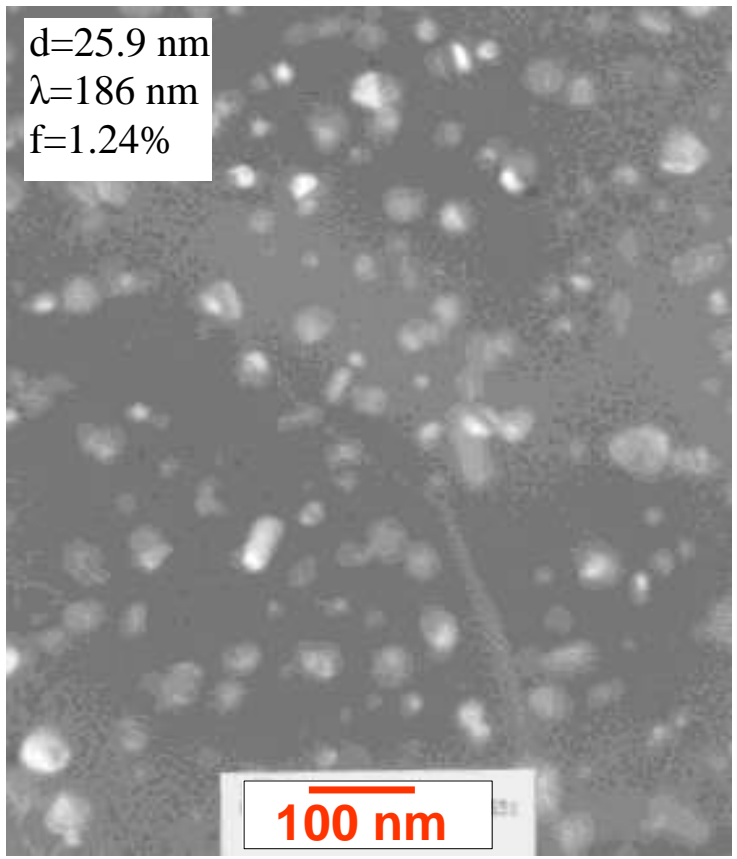

(c)

Figure 6. TEM micrographs of Al-Ge-Si, aged at $200{ }^{\circ} \mathrm{C}$ for: (a) $0.5 \mathrm{~h}(\mathrm{UA})$, (b) $12 \mathrm{~h}$ (PA), and (c) one week (OA). 


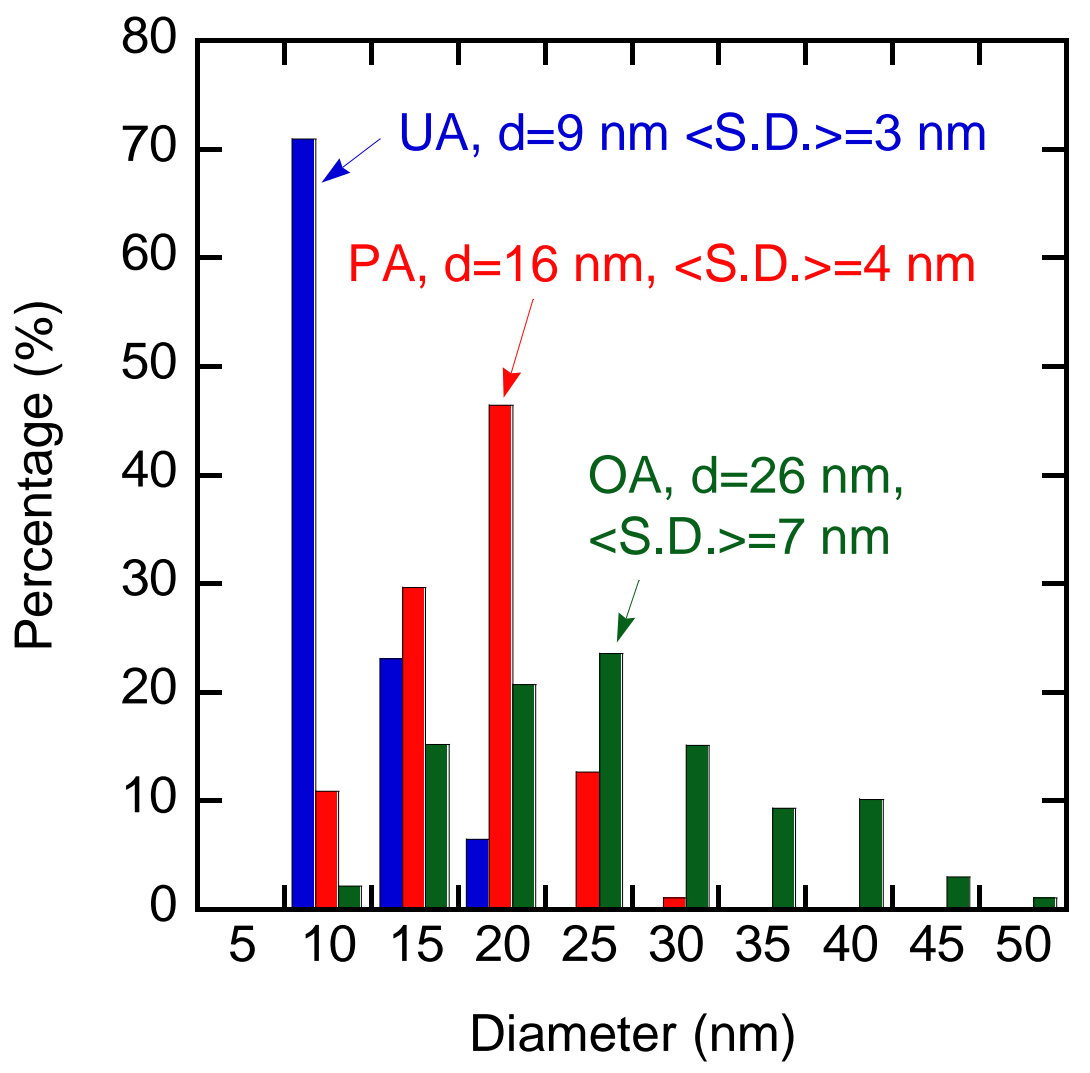

Figure 7. Precipitate size distribution of Al-Ge-Si aged at $200^{\circ} \mathrm{C}$. The average diameter (d) and standard deviation are included.

Corresponding aging information for all aging temperatures is summarized in Figure 8. As expected, the average precipitate size for OA is larger than for PA. Each label contains three numbers, $d$ (average precipitate diameter), $\lambda$ (average precipitate spacing) and $f$ (precipitate volume fraction). The PA materials at various temperatures have similar precipitate characteristics and the volume fractions approach the limit of $1.25 \%$. That is, all Si and Ge are out of solution by the time that the PA condition is reached. 


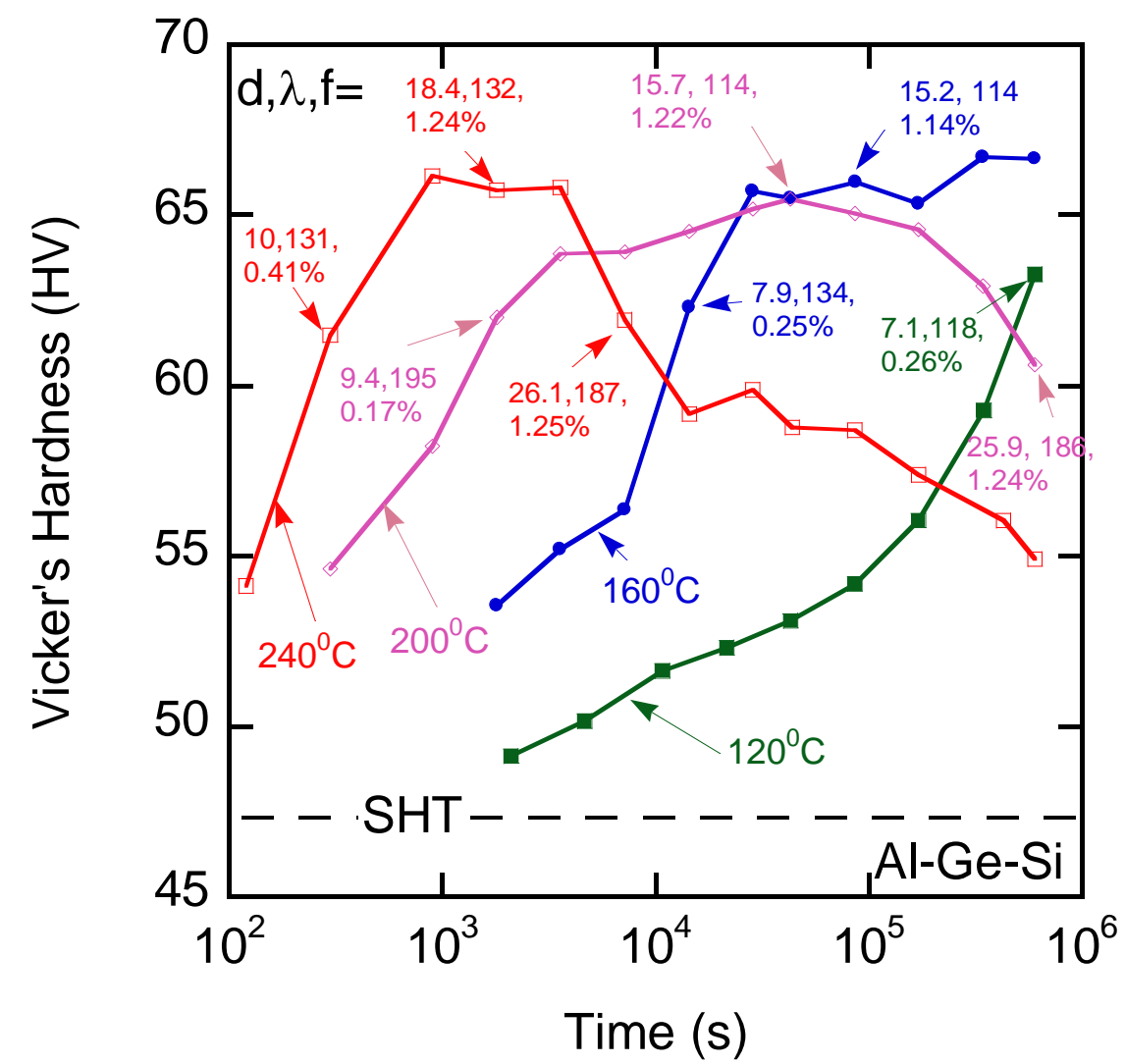

Figure 8. Aging curves for $\mathrm{Al}-\mathrm{Ge}-\mathrm{Si}$ with quantitative precipitate measurement results.

The precipitate structure parameters are plotted in Figures 9 (a) - (d) with respect to $\theta$, a time quantity adjusted for aging temperature that is defined as follows:

$$
\theta=\mathrm{t} * \exp \left(-\frac{\Delta \mathrm{G}}{\mathrm{RT}}\right)
$$

where $\mathrm{t}$ is the aging time, $\mathrm{T}$ is the aging temperature, $\mathrm{R}$ is the gas constant and $\Delta G$ refers to the activation energy which equals to $117 \pm 5 \mathrm{~kJ} / \mathrm{mol}$ for diffusion of $\mathrm{Ge}$ or $\mathrm{Si}$ in aluminum [86-88]. Figures 9 (a) - (d) illustrate aging of this alloy as follows:

- All of the curves collapse to a master curve for all tested temperatures and times, Figures 9 (a) - (d).

- The precipitate size increases monotonically with $\theta$ (time and temperature), Figure 9 (b). The SHT data point (black filled circle) corresponds to aging for two weeks at room temperature after solution heat treatment and quenching.

- The spacing of precipitates is at a minimum at the PA condition. It is approximately equal in the UA and OA conditions, Figure 9 (c).

- The volume fraction of precipitates increases abruptly near the PA condition where $\theta$ is $\sim 10^{-13} \mathrm{~h}$, saturating at the thermodynamically expected value, Figure 9 (d). 


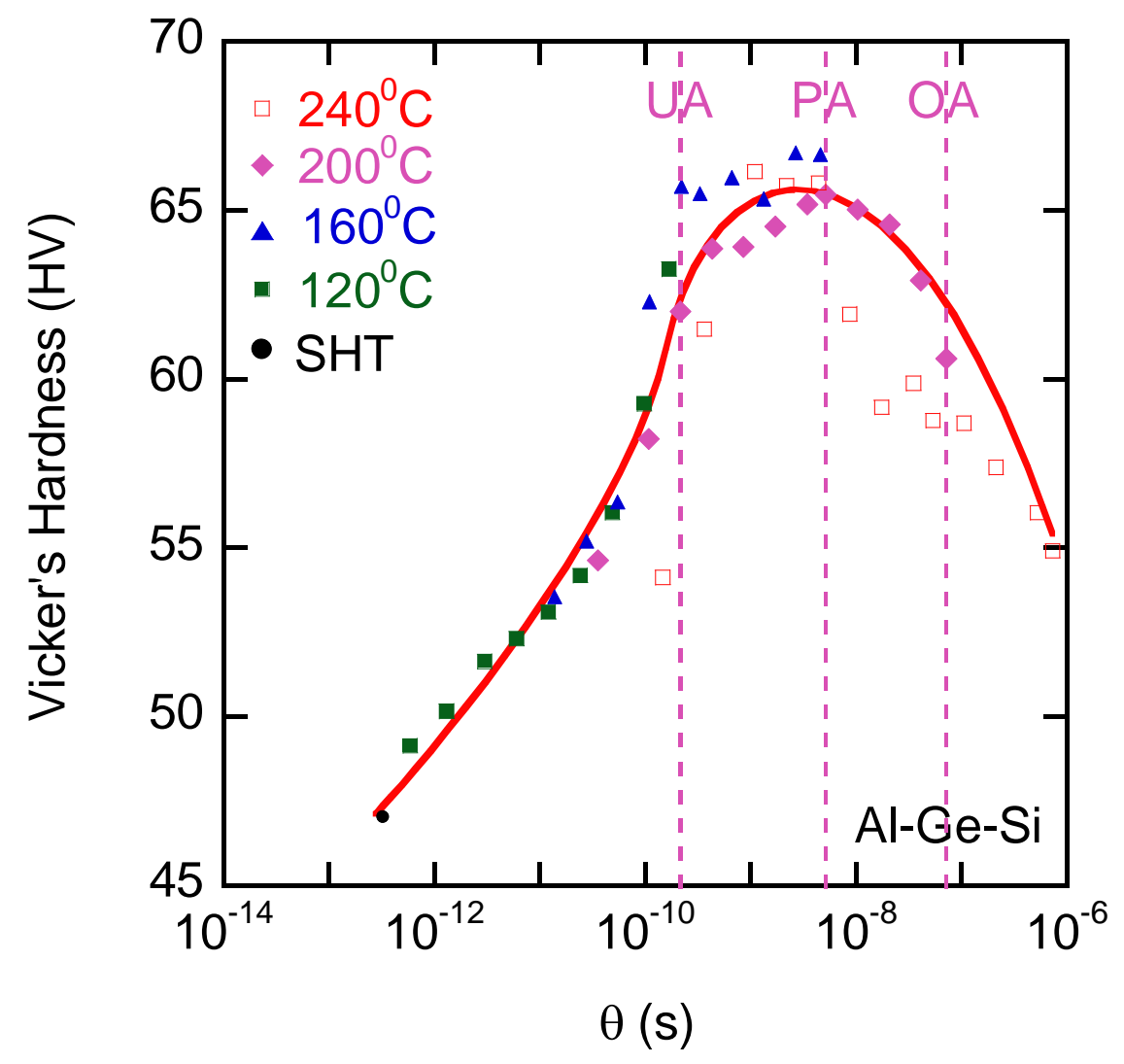

(a) 


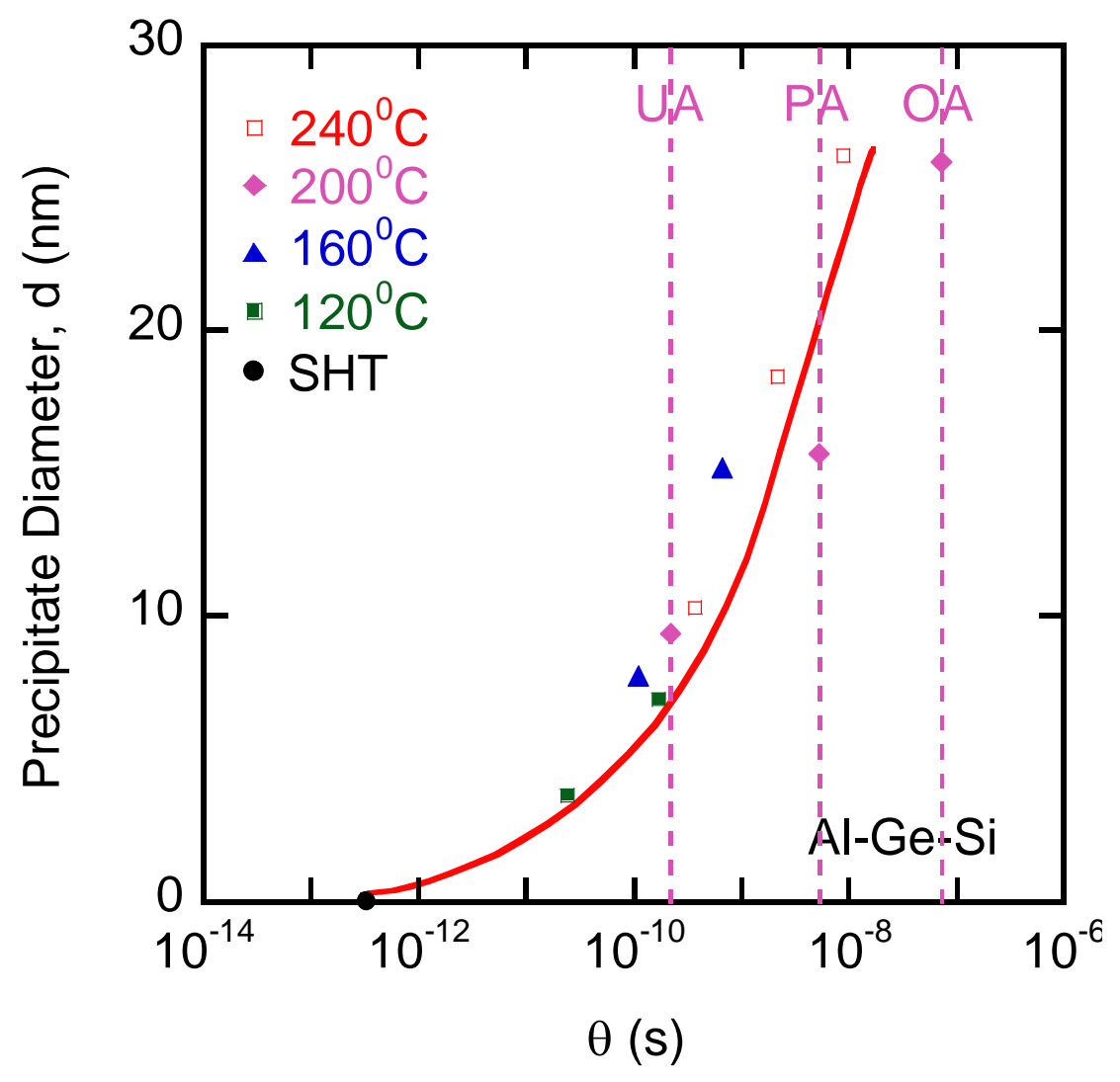

(b) 


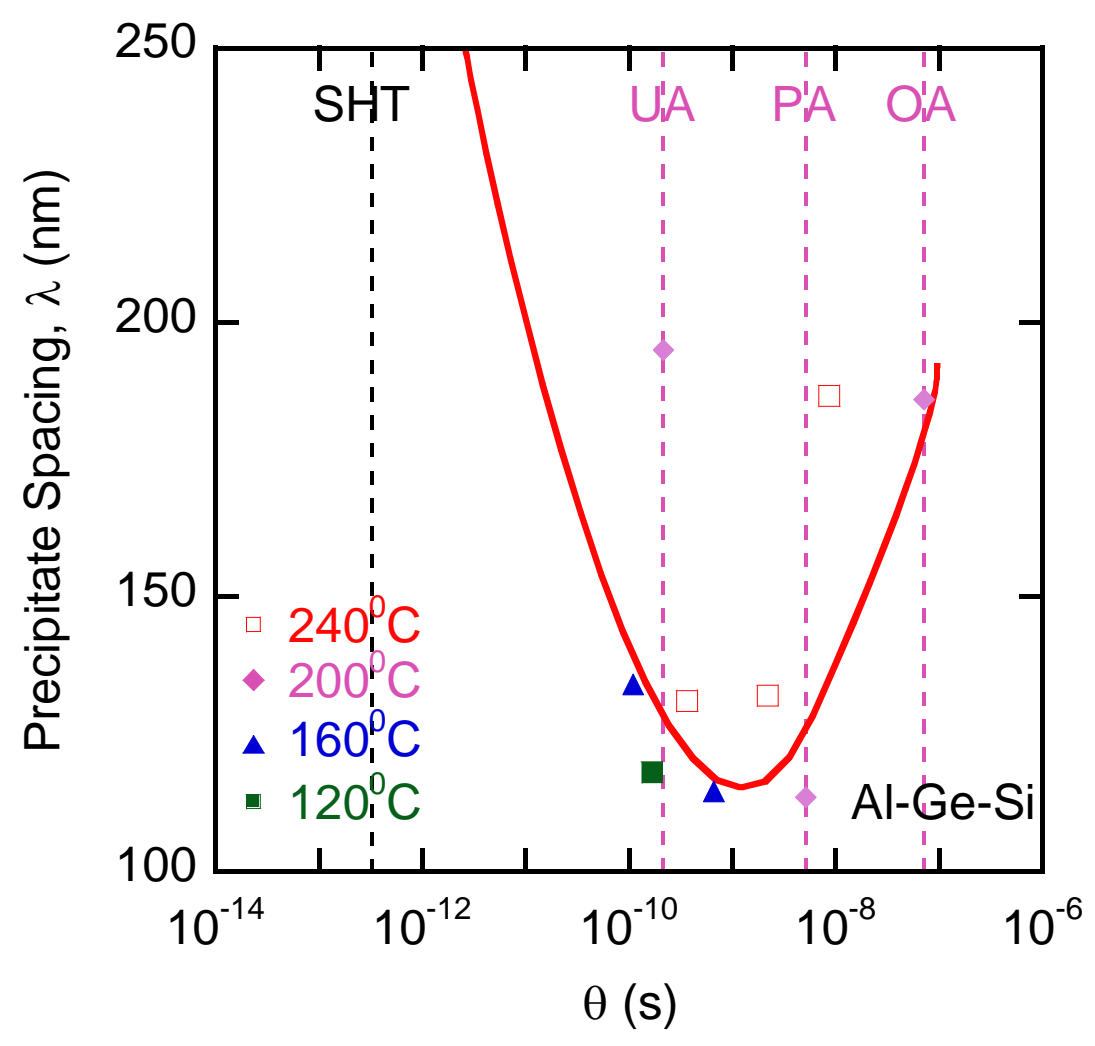

(c) 


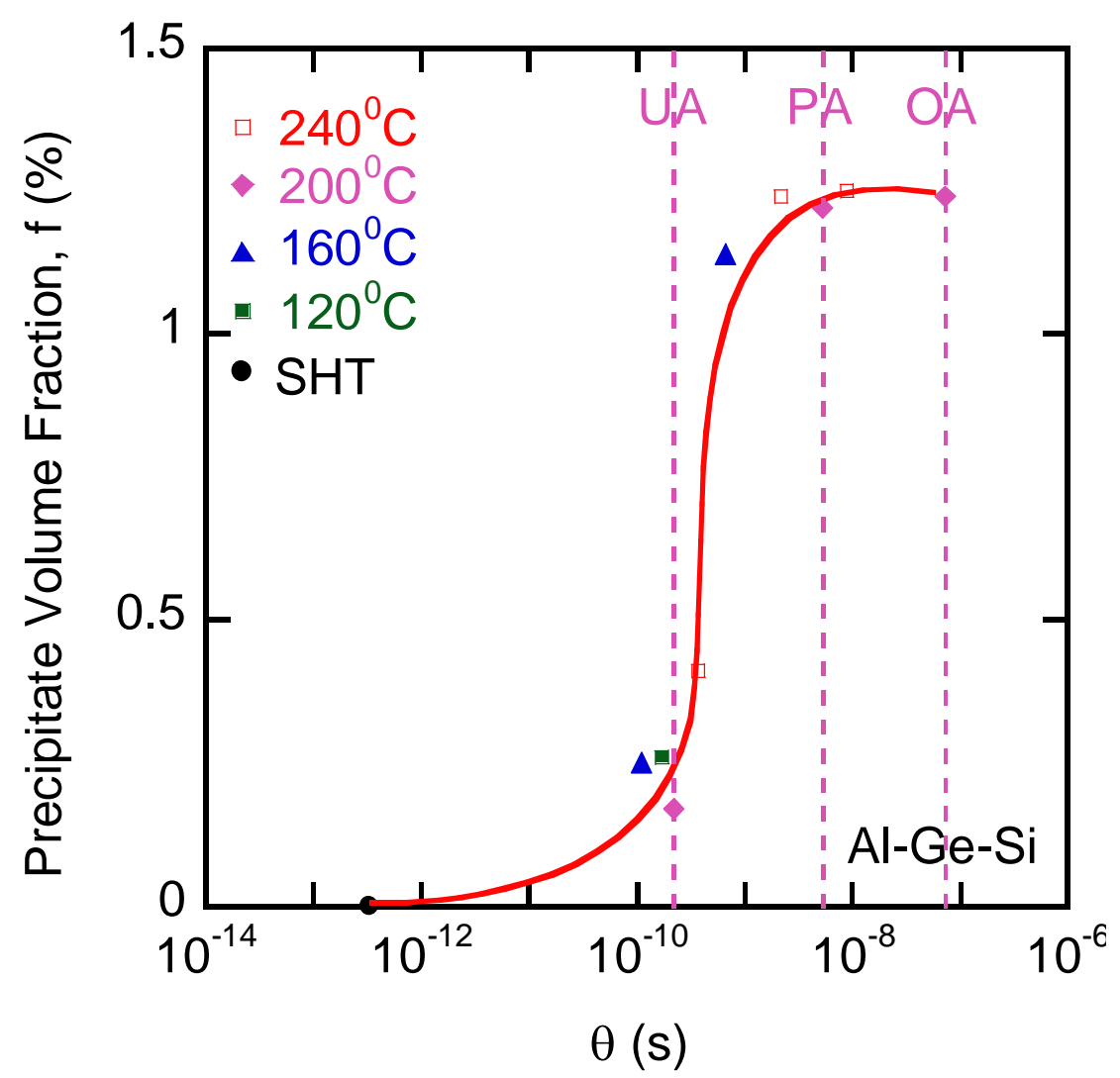

(d)

Figure 9. Evolution of precipitate characteristics: (a) the master aging curve, (b) size, (c) spacing, and (d) volume fraction.

\subsection{Tensile results}

The engineering stress strain curves for the UA, PA, OA, and SHT conditions are shown in Figure 10, along with that for annealed pure aluminum [89] for comparison. The high UA strain hardening is presumably related to the absence of precipitates, which tend to increase the yield stress and reduce high-strain strain hardening [64,90]. SHT and pure Al are similarly devoid of precipitates but the SHT exhibits solid-solution strengthening 9 .

The Orowan bypass mechanism (for hard pins such as Ge/Si particles of the sizes seen here - see Introduction) associates the yield stress increase from aging to particle spacing $[30,31,91]$ as follows:

$$
\sigma=\sigma_{o}+\alpha^{\prime} \mu b / L
$$

(Equation 6)

9 The SHT condition shows serrated flow. Although the serrated flow in Al alloys is complex and can be influenced by both solutes and precipitates [122,123], to the first approximation higher solute content $\mathrm{s}$ correspond to the appearance Portevin-Lechatelier Effect, as expected mechanistically. The SHT condition has, of course, the highest concentration of solutes. 
where $\sigma_{o}$ is the yield stress with no precipitates, $\alpha$ ' is a constant on the order of unity, $\mu$ is the shear modulus, $b$ is the Burger's vector and $L$ is the particle spacing. The shear modulus for aluminum is $26 \mathrm{GPa}$, and its Burger's vector is $2.86 \times 10^{-10} \mathrm{~m}$.

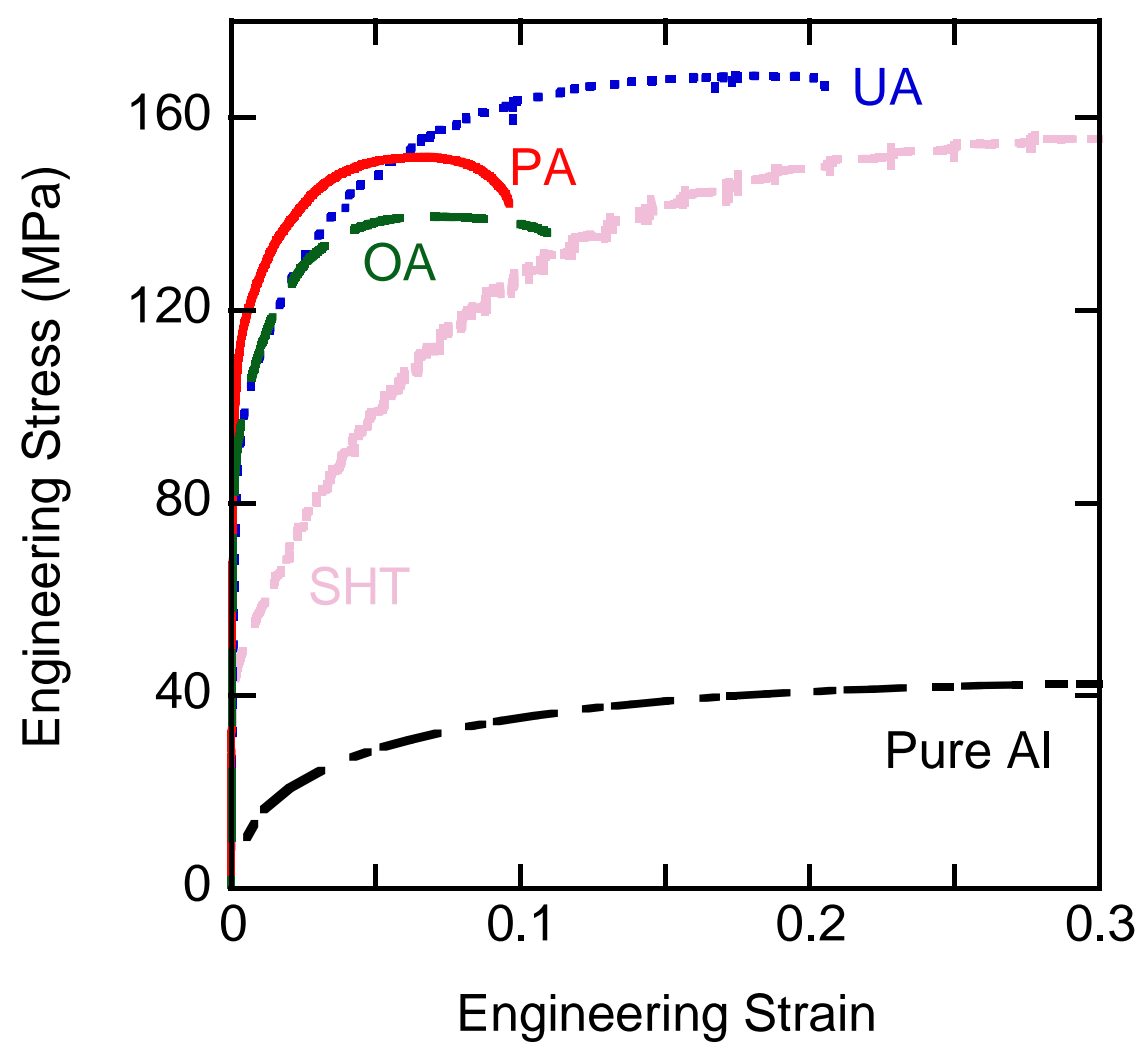

Figure 10. Tensile curves of Al-Ge-Si aged at $200^{\circ} \mathrm{C}$, as compared to pure $\mathrm{Al}$.

Table 3 applies Orowan analysis using Equation 6 to the UA, PA, and OA conditions, starting from the SHT condition with the assumption that particle spacing is infinite for the SHT condition. $\quad \alpha^{\prime}$ is a fit parameter $(=1.22)$ such that the predicted yield stress for the UA condition agrees with the measurement; using this same value the strengths of the PA and OA conditions are predicted as shown. It is shown that the measured $\left(\sigma_{\text {meas }}\right)$ and predicted $\left(\sigma_{\text {pred }}\right)$ yield stresses agree reasonably well. This confirms the hard pin nature of the precipitates (i.e. as embodied in Equation 6) in agreement with the smallest precipitates being much larger than the size where shearing is possible $(6 \mathrm{~nm}$ in UA, 12 $\mathrm{nm}$ in PA, and $15 \mathrm{~nm}$ in OA vs. 1.2-2.3 nm calculated using Equation 1).

Table 3. Measured and predicted yield stresses of aged Al-Ge-Si

\begin{tabular}{|l|c|c|c|}
\hline & $\sigma_{\text {meas }}(\mathrm{MPa})$ & $\sigma_{\text {pred }}(\mathrm{MPa})$ & $\sigma_{\text {meas }} \sigma_{\text {pred }}(\mathrm{MPa})$ \\
\hline SHT & 46.9 & $/$ & $/$ \\
\hline UA & 93.5 & $93.5($ fit $)$ & 0 \\
\hline PA & 114.6 & 126.9 & -12.3 \\
\hline
\end{tabular}




\begin{tabular}{|l|l|l|l|}
\hline OA & 96.4 & 95.9 & 0.5 \\
\hline
\end{tabular}

Table 3 also shows that the yield stress is maximized for the PA condition, consistent with the hardness measurement, and the OA and UA conditions have approximately the same, lower yield stress, also consistent with hardness.

The strain hardening rates of the aged materials are plotted against strain and $\sigma-\sigma_{y}$ in Figures $11 \mathrm{a}$ ) and (b). Most remarkable is the identical strain hardening behavior of the OA and PA conditions, which is significantly different from the UA condition. That is, strain hardening behavior develops during aging, but does not reverse upon overaging in lockstep with hardness and yield stress. The extended strain hardening range for the UA condition is consistent with observations that solutes assist in the multiplication of dislocations over large strain ranges $[64,90]$.

In order to characterize the strain hardening as a function of solute content, the stress-strain curves in the SHT condition (i.e. precipitate-free, fully solid solution strengthened) of $\mathrm{Al}-1 \% \mathrm{Ge}-\mathrm{Si}$ and $\mathrm{Al}-0.4 \% \mathrm{Ge}-\mathrm{Si}$ were compared with that of the pure Al. The change of yield stress from solid solution strengthening is typically described by

$$
\sigma_{\mathrm{ss}}=\mathrm{KC}^{\alpha}
$$

where $\alpha$ is constant [92-94]. To extend this same formalism to the solute effect on strain hardening, the constant $\mathrm{K}$ was modified by a Voce type strain hardening law as follows:

$$
\sigma_{\mathrm{ss}}=\{\mathrm{a}-\mathrm{b} \times \exp (-\mathrm{c} \varepsilon)\} \mathrm{C}^{2 / 3}
$$

Optimized parameters were obtained as described in the Appendix A, as follows $a=2.6$ $\mathrm{GPa}, \mathrm{b}=2.6 \mathrm{GPa}$ and $\mathrm{c}=8$ for the dilute $\mathrm{Al}-\mathrm{Ge}-\mathrm{Si}$ alloys tested in the current work. This seems to be the first time that the entire strain-hardening curve was expressed as a simple function of solute content. As shown in the Appendix A, with further analysis this equation is converted to expressing the evolution of dislocation density as a function of solute concentration, also a first. 


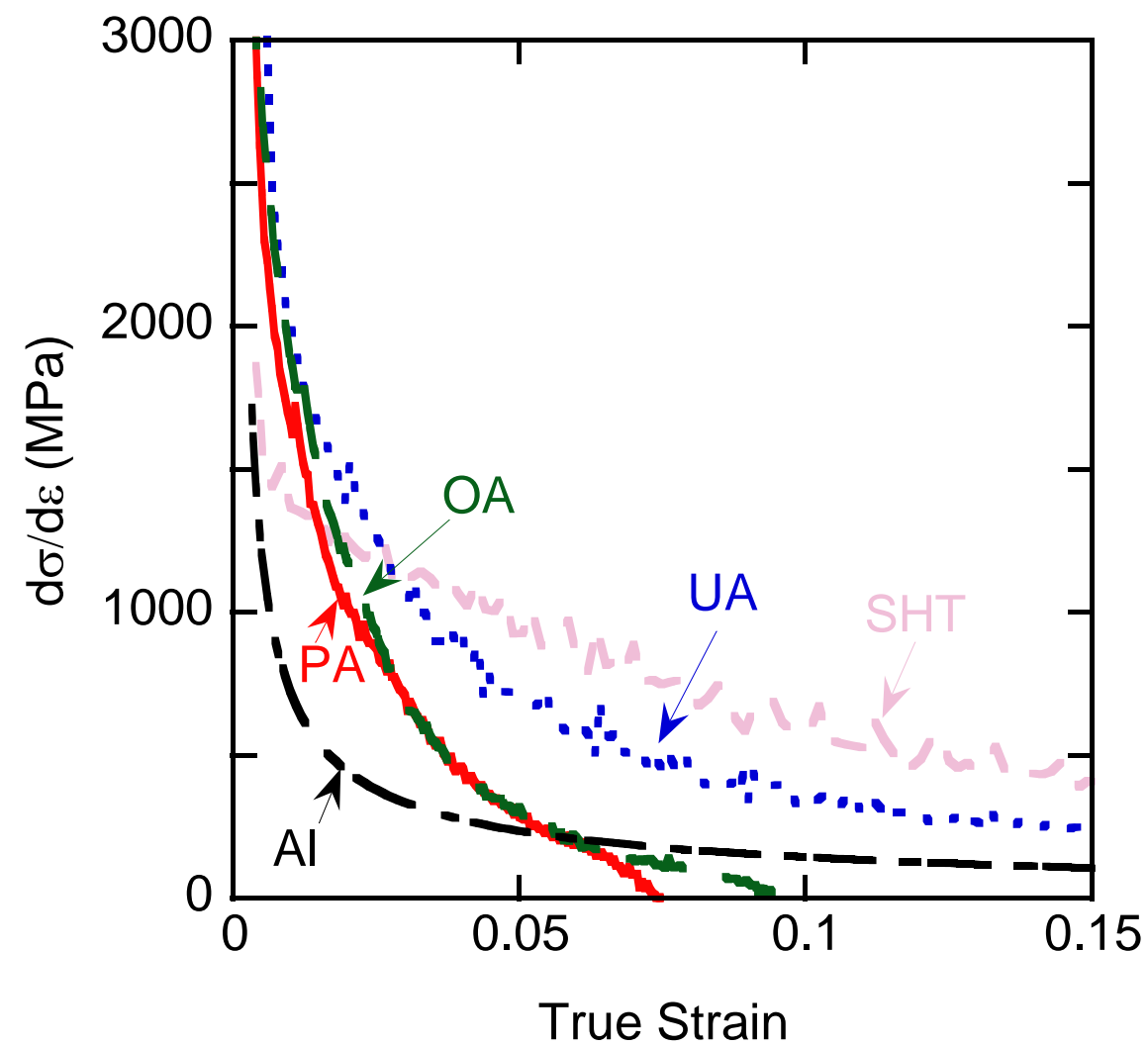

(a) 


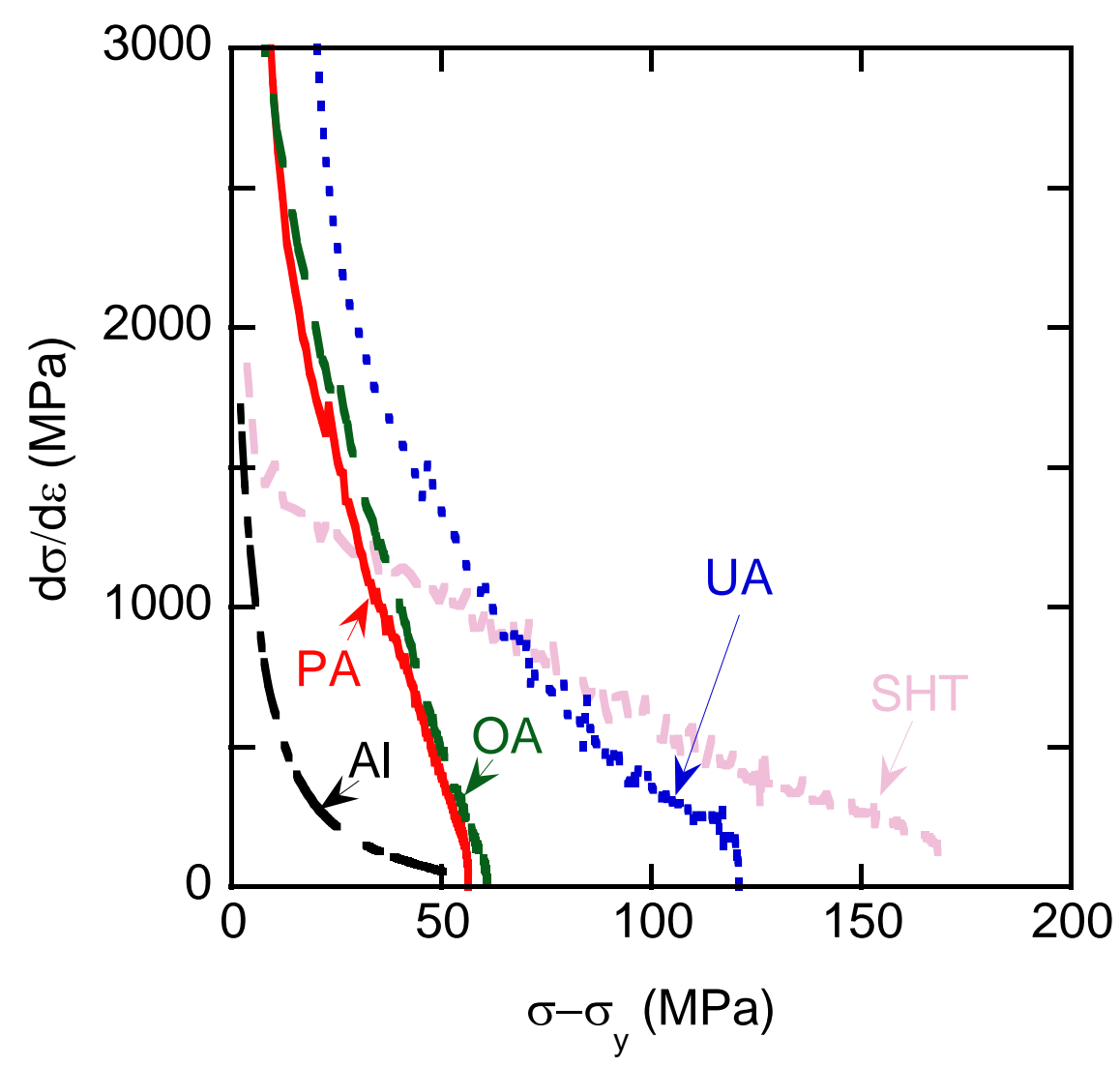

(b)

Figure 11. The evolution of work hardening rate of Al-Ge-Si aged at $200^{\circ} \mathrm{C}$, as compared to pure aluminum: (a) d $\sigma / \mathrm{d} \varepsilon$ vs. $\varepsilon$ and (b) $\mathrm{d} \sigma / \mathrm{d} \varepsilon$ vs. $\sigma-\sigma_{\mathrm{y}}$.

\subsection{Compression-Tension results}

The Bauschinger factors, i.e., $\beta, \gamma$, and $\Delta E$ for aged Al-Ge-Si and for annealed pure aluminum $^{10}$ are plotted as a function of aging time in Figures 12 (a)-(c). The over-over-aged condition (OOA) in these figures refers to the aging condition of two weeks at $160^{\circ} \mathrm{C}$ plus one week at $240^{\circ} \mathrm{C}$. For the SHT condition and for annealed pure Al, arbitrary aging times were assigned in order to plot the data together with the other, normally-characterized conditions.

10 Some of the original stress-strain data was lost after the original experiments were performed, thus making impossible the consistent calculation of some Bauschinger Factors for some cases. The special nature of the custom-made materials does not allow for new testing under matching conditions. 


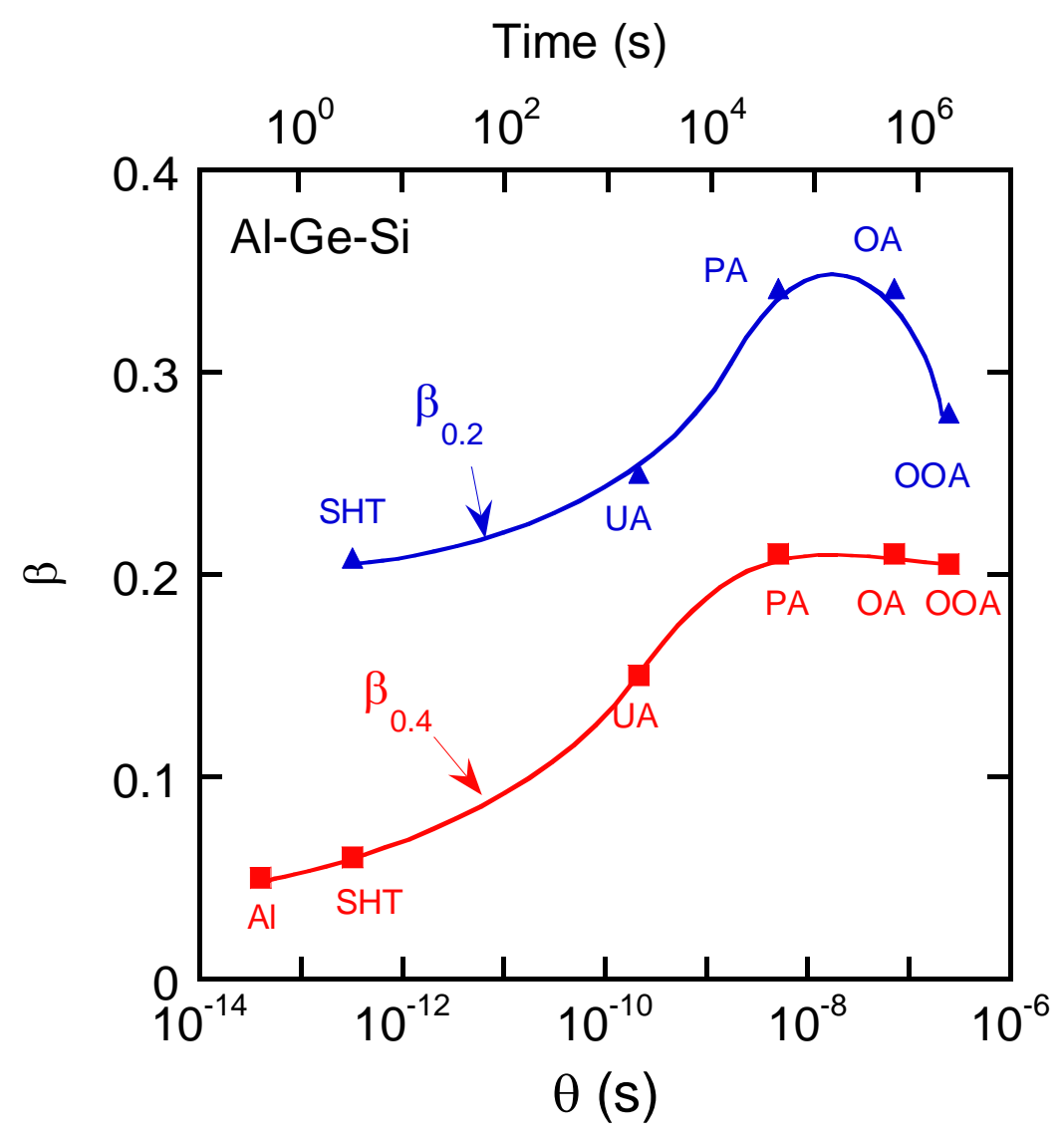

(a) 


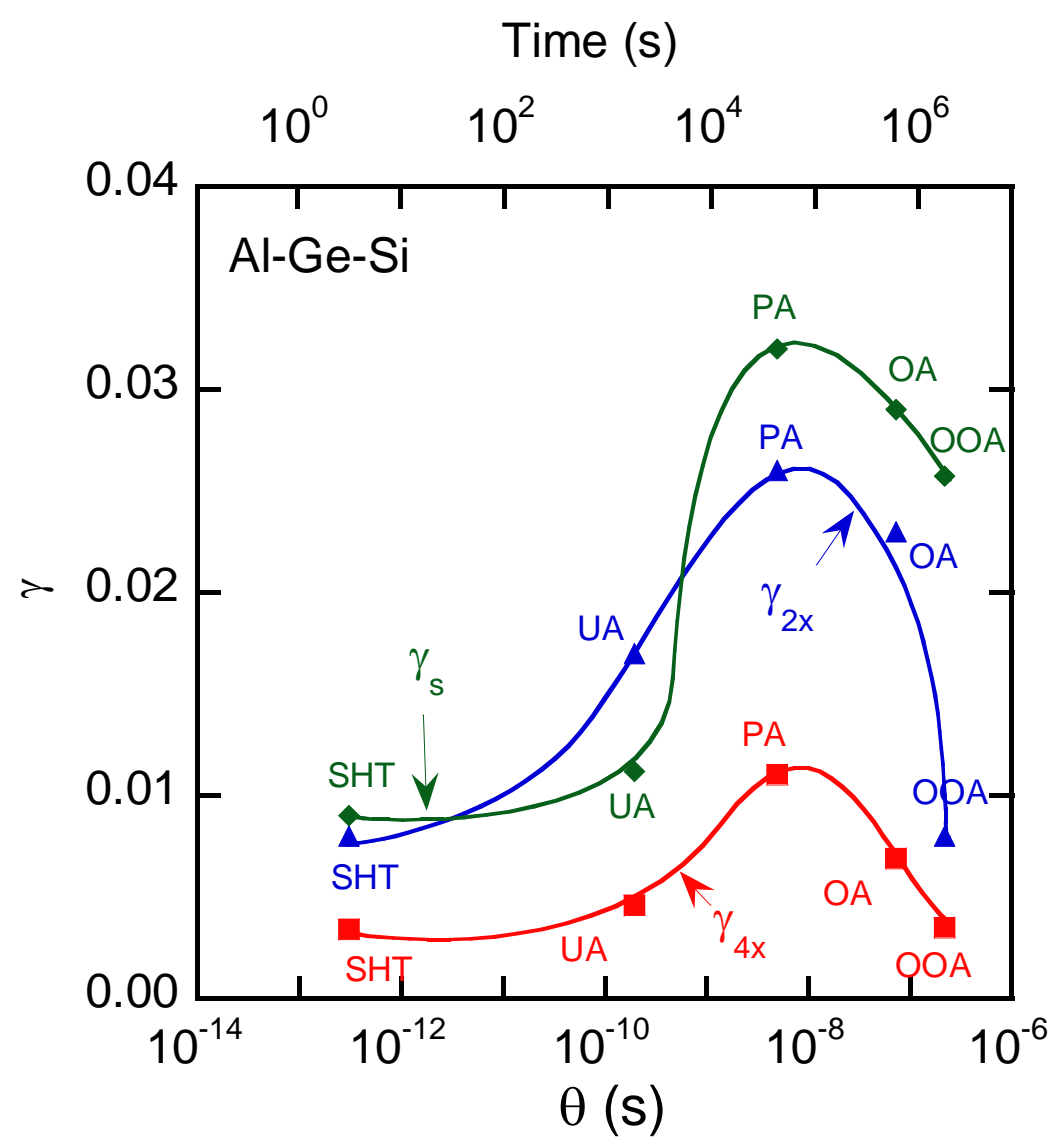

(b) 


\section{Time (s)}

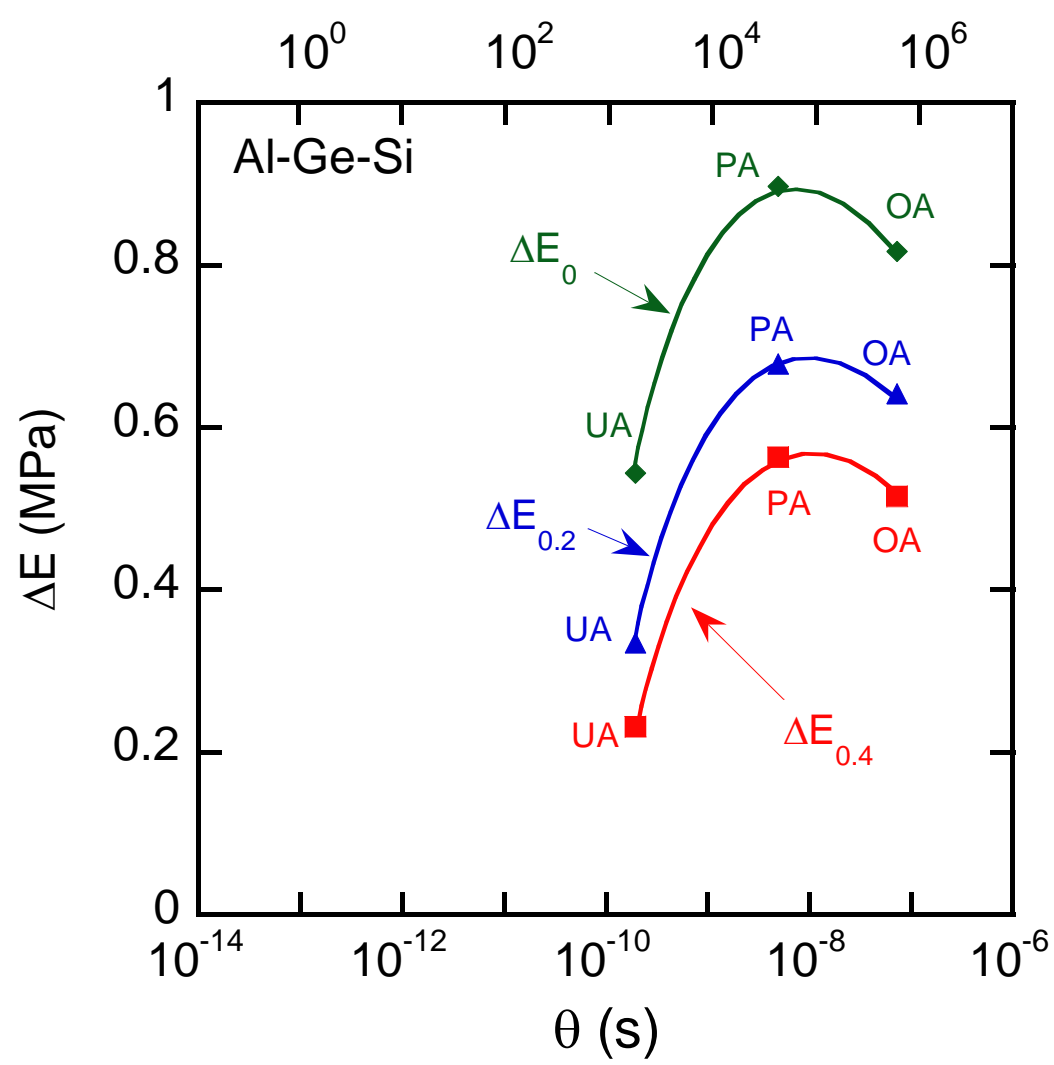

(c)

Figure 12. Measured "Bauschinger Factors" of pure aluminum and Al-Ge-Si materials at SHT, UA, PA and OA conditions (a) Bauschinger Stress Factor, $\beta$ (b) Bauschinger Strain Factor, $\gamma$ and (c) Bauschinger Energy Factor, $\Delta \mathrm{E}$ (See Section II.D for definitions).

In general, Figures 12 show that all characteristics of the Bauschinger effect are maximized when the strength is maximized, that is, at the PA condition, consistent with several other $\mathrm{Al}$ alloys [57,58]. However, it is clear that the Bauschinger effect declines very little in the OA condition. The Bauschinger effect for OA is similar to PA, much stronger than for the UA condition, contrary to tensile measures of hardness and yield stress. The value of $\beta$ determined using the $0.4 \%$ offset criterion and some of the $\Delta E$ results maintain the PA value throughout the OA and even into the OAA conditions, consistent with some reports in the literature $[62,63]$.

Another striking factor is the similarity of $\beta$ values for pure Al and the SHT alloy condition, particularly in view of the large disparity of flow stresses; these values are consistent with forward and reverse torsion results from the literature [95]. The lack of effect of solid solution strengthening on the magnitude of the Bauschinger effect is consistent with a dislocation pile-up or bow-out mechanism: solutes are not hard pins and therefore do not induce dislocation pinning or looping. 
The Bauschinger Stress Factor $\beta$ is shown in Figure 12 (a). The variation of $\beta_{0.2}$ corresponds roughly that of precipitate spacing or, equivalently, yield stress. The variation of $\beta_{0.4}$ corresponds more closely with volume fraction of precipitate. The Bauschinger Strain Factor $\gamma$ is presented in Figure 12 (b). All three of the strain-magnitude measures are qualitatively similar: they increase initially from SHT to UA and PA, but drop after PA. The Bauschinger Energy Factor $\Delta E$ is shown in Figure 12 (c). The results are consistent with the results of $\beta$ with the development of $\Delta E$ apparently related to both precipitate spacing and precipitate volume fraction.

Table 4 summarizes the Bauschinger Factors $\beta, \gamma$, and $\Delta E$, for some definition choices. The UA material has a similar particle spacing as the OA case (as expected from the same hardness and yield stress), but all characterization parameters of the Bauschinger effect are significantly smaller in UA as compared to the equal-strength OA. The PA and OA conditions have similar Bauschinger factors even though the precipitate spacing differs by $60 \%$. These observations suggest that the Bauschinger factors in a stable population of non-shearable precipitates are controlled both by the precipitate spacing, and by the volume fraction of precipitates.

Table 4 Bauschinger variables for aged Al-Ge-Si materials

\begin{tabular}{|l|c|c|c|c|c|c|c|}
\hline & $d(\mathrm{~nm})$ & $\lambda(\mathrm{nm})$ & $f(\%)$ & $\beta_{0.2}$ & $\beta_{0.4}$ & $\gamma$ & $\Delta E_{0}$ \\
\hline SHT & - & - & - & 0.21 & 0.06 & 0.008 & - \\
\hline UA & 9.4 & 195.3 & 0.2 & 0.25 & 0.15 & 0.017 & 0.54 \\
\hline PA & 15.7 & 113.7 & 1.2 & 0.34 & 0.21 & 0.026 & 0.90 \\
\hline OA & 25.9 & 185.9 & 1.2 & 0.34 & 0.21 & 0.023 & 0.82 \\
\hline
\end{tabular}

The reverse hardening behavior of aged $\mathrm{Al}-\mathrm{Ge}-\mathrm{Si}$ is compared with that of commercial $\mathrm{Al}$ 2524 alloy in Figures 13, in terms of $\Delta \sigma$ vs. $\Delta \varepsilon$, where $\Delta \sigma=\left|\sigma_{\text {monontonic }}\right|-\left|\sigma_{\text {reverse }}\right|$, and $\Delta \varepsilon$ is the absolute strain from the zero-stress point following the reversal. Such a plot is convenient to reveal the transition region with more resolution. The difference among the aging conditions for the commercial alloy is much greater in view of the greater alloy content. Figure 13 (a) shows that the OA sample for Al-Ge-Si experienced nearly the same Bauschinger transition as the PA sample, although its precipitate size and spacing are 60\% larger. Figure 13 (b) [63] shows that the OA condition for Al 2524 has a larger Bauschinger effect than the PA condition. This contradicts to the Orowan theory of the Bauschinger effect $[31,94]$, where the precipitate spacing would be the only controlling parameter for dilute hard-pin precipitates. 


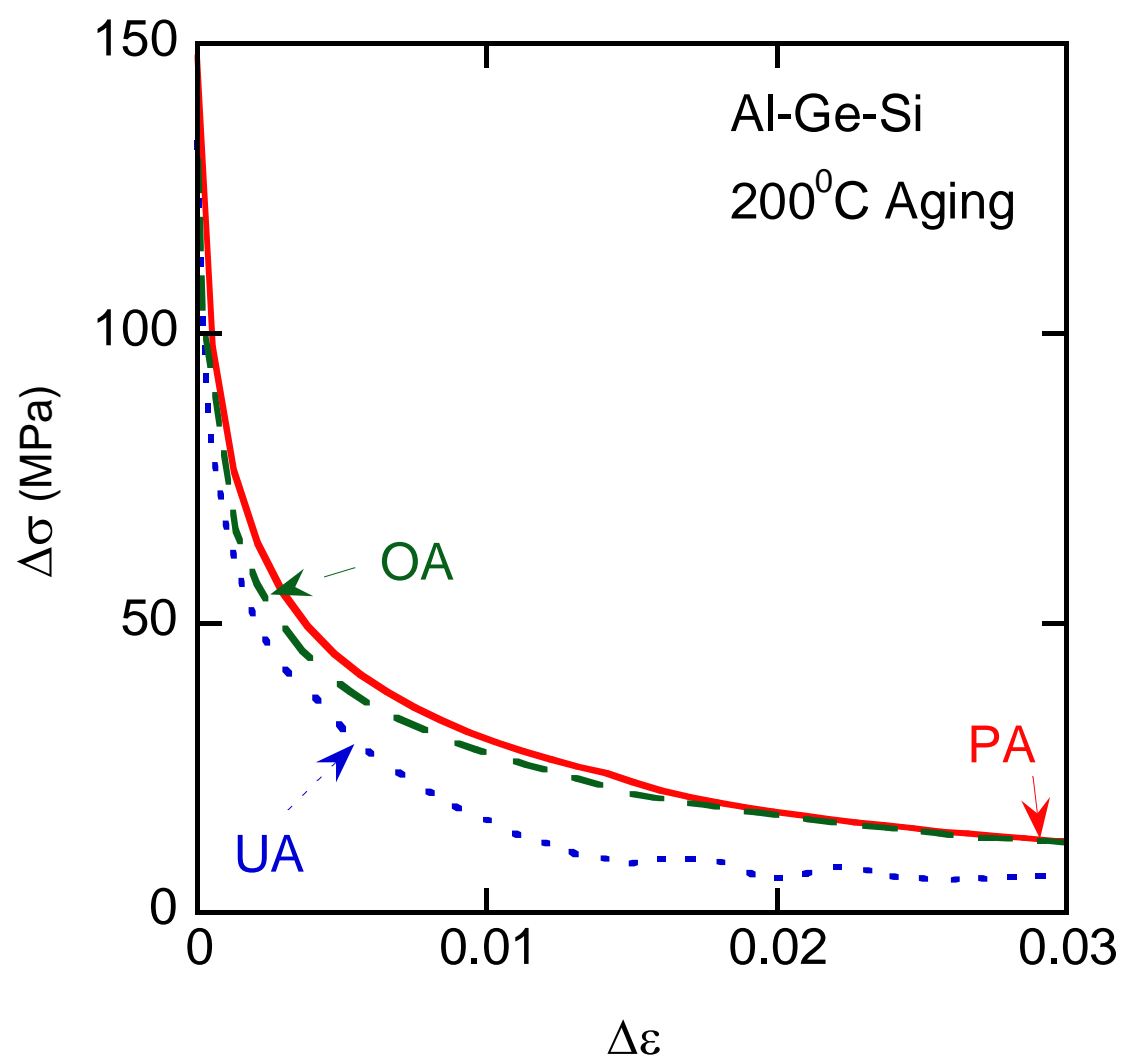

(a) 


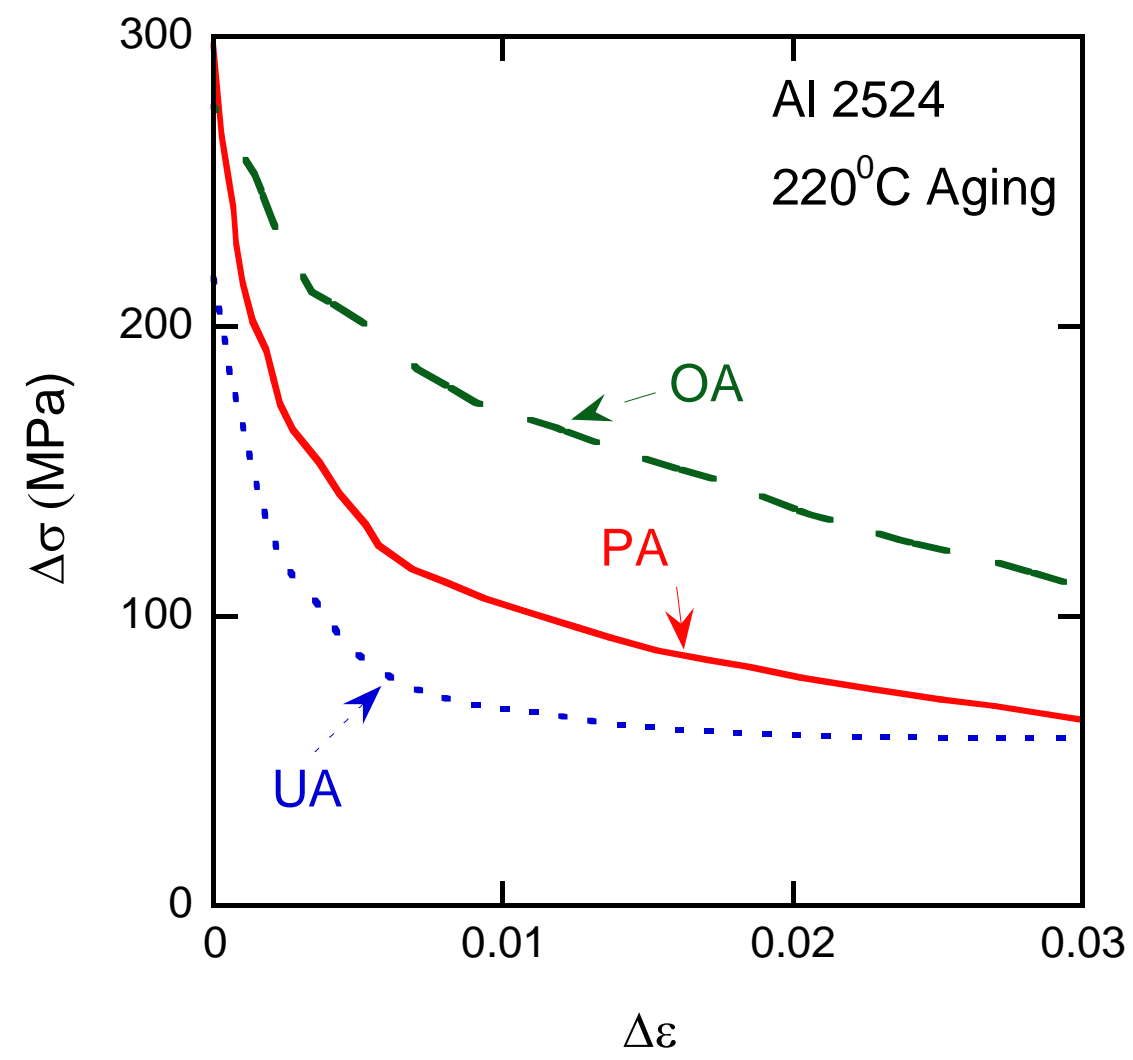

(b)

Figure 13. Reduced CT hardening curves for various aging conditions: (a) Al-Ge-Si, and (b) Al 2524 [83]

Any factor that causes heterogeneous deformation inside of the sample can contribute to the Bauschinger effect [96,97]. For the aged Al-Ge-Si materials, the only plausible sources of significant inhomogeneity are precipitates, grain misorientations, and grain boundaries. The results for these alloys show that precipitates, when present, are the main source of the Bauschinger effect, even for these dilute alloys. The precipitation during aging triples the magnitude of the Bauschinger effect as compared with the SHT condition (see Table 4). This dominance of precipitates in the Bauschinger effect for $\mathrm{Al}-\mathrm{Ge}-\mathrm{Si}$ is consistent with theories and observations for hard pins from the literature $[45,57,58,98]$.

\section{SIMULATIONS AND DISCUSSION}

The experiments and analyses presented above show that non-shearable precipitates are the predominant source of the Bauschinger effect, even in these dilute alloys. However, the results also show that the Bauschinger effect is controlled not only by particle spacing, as for example an Orowan mechanism would dictate, but also by particle size and volume 
fraction, which vary together during pre-peak aging, but which can be distinguished after peak aging, when the volume fraction is constant but the precipitate size continues to grow and the particle spacing decreases slowly.

As introduced in Section I, most attempts to rationalize the Bauschinger effect rely on continuum models of inhomogeneity [23-28] and semi-phenomenological single-dislocation models [19,20,52,53] including dislocation bow-out models [34-36]. In the current work, these ideas will be tested by constructing models of the measured microstructures and using these to simulate and predict the mechanical behavior.

\subsection{Representative Volume Elements (RVE's)}

RVEs were constructed to correspond to the quantified microstructures for three aging conditions: UA, PA, and OA. Figure 14 (a) shows a cross-sectional image revealing an $\mathrm{Al}$ matrix and evenly-distributed elastic Ge-Si spherical particles chosen to represent approximately the measured size distributions, spacings, and volume fractions as presented in Table 4 and Figures 7 and 8.

For the UA, PA, and OA conditions, the dominant source of the hardening and Bauschinger effect is the precipitates. Therefore, no attempt was made to reproduce grains in these simulations, instead a single-crystal matrix was modelled. Including grains would require modeling a wide range of scales that would be prohibitive in terms of RVE size. The approach is further justified by the fact that the Hall-Petch effect is insignificant or minimal for $\mathrm{Al}$ alloys having normal grain sizes. The Hall-Petch slope or Al alloys is $<0.1 \mathrm{MN} / \mathrm{m}^{-3 / 2}[89,99]$. 


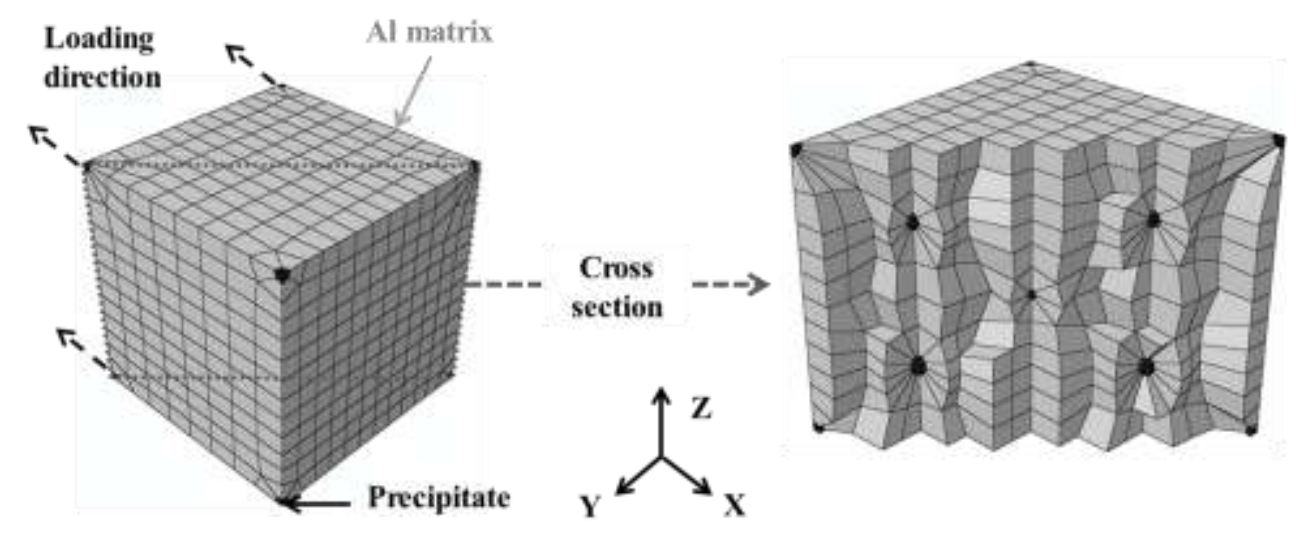

\begin{tabular}{|c|c|c|c|}
\hline & \multicolumn{3}{|c|}{ Number of particles } \\
\hline Particle diameter $(\mathrm{nm})$ & $\begin{array}{c}\mathrm{UA} \\
(\mathrm{f}=0.2 \% / \lambda=243 \mathrm{~nm})\end{array}$ & $\begin{array}{c}\mathrm{PA} \\
(\mathrm{f}=1.2 \% / \lambda=132 \mathrm{~nm})\end{array}$ & $\begin{array}{c}\text { OA } \\
(\mathrm{f}=1.2 \% / \lambda=198 \mathrm{~nm})\end{array}$ \\
\hline 10 & 7 & 1 & 2 \\
\hline 15 & 2 & 3 & 2 \\
\hline 20 & 1 & 5 & 2 \\
\hline 25 & - & 1 & 1 \\
\hline 30 & - & - & 1 \\
\hline 35 & - & - & 1 \\
\hline 40 & - & - & 1 \\
\hline
\end{tabular}

(a)

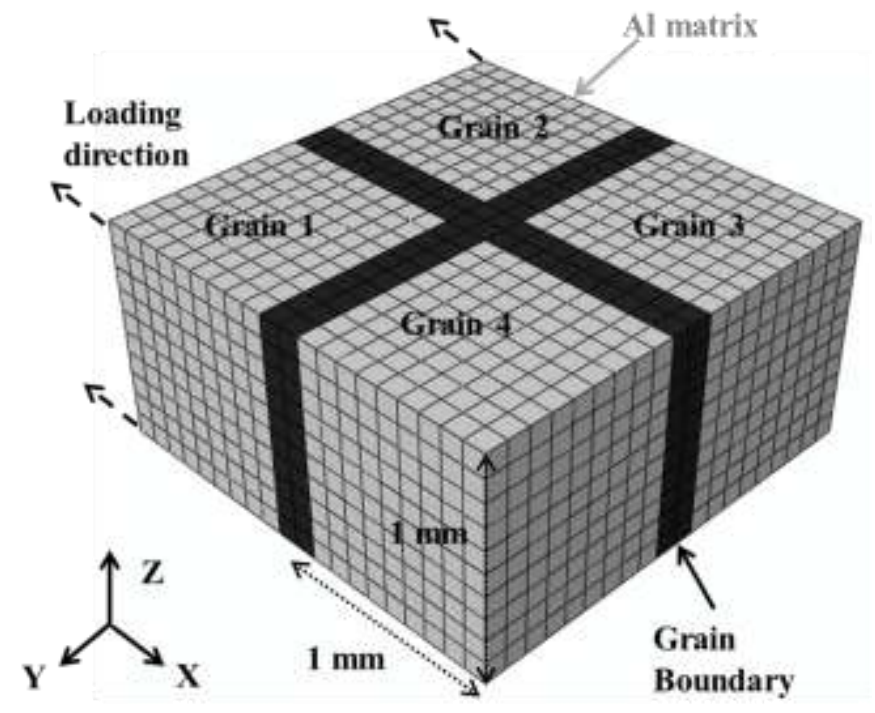

(b)

Figure 14. Details of an new-RVE/FE model of Al-Ge-Si alloy for (a) UA/PA/OA conditions with $\mathrm{Al}$ matrix and embedded hard particles as shown in the accompanying table to reproduce the measured precipitate properties, and (b) Pure Al/SHT condition with 4 grains and corresponding grain boundaries. 
The pure Al and SHT conditions have no precipitates, so it becomes practical computationally, and necessary for accuracy, to include the possibly-significant grains (grain size of $1 \mathrm{~mm}^{11}$ ) in the mechanical simulations. Figure 14 (b) shows the grain arrangement modelled by the RVE's for the pure Al and SHT condition of the Al-Ge-Si (the only difference being the flow strength of the matrix by solid solution hardening).

For all mechanical simulations, symmetry boundary conditions ${ }^{12}$ were applied to the $\mathrm{x}^{-}$, $\mathrm{y}-$, and $\mathrm{z}$ - planes. The bonding between the elastic particle and the matrix is assumed to be perfect i.e., no interface separation or sliding is allowed. A displacement boundary condition is applied to the loading direction.

\subsection{Constitutive Models}

Three constitutive models were developed corresponding to three assumed precipitate-based mechanisms governing the Bauschinger effect: 1) a standard continuum model, 2) a standard Crystal Plasticity (CP) model based on slip system geometry [100-104] and 3) a novel Super Dislocation (SD) model essentially similar to the CP model but accounting for the discrete dislocation-dislocation, dislocation-obstacle elastic interactions ${ }^{13}$.

The SD model incorporates real length scales in the form of the Burgers vector and precipitate size (or, equivalently, spacing). It does not include any arbitrarily determined or fit constitutive parameters [105] such as material length scale in a strain-gradient model [106-108]. In contrast, as will be noted below, the volume fraction is the only important microstructural variable for the continuum and the CP model adds a texture dependence to that. The precipitates are modelled to exhibit no plastic deformation.

Continuum Model - The Al matrix in the continuum model is elastically-isotropic with Young's modulus of E $=70 \mathrm{GPa}$ and Poisson's ratio of $v=0.3$, and having a plastic response obeying a von-Mises isotropic yield function and an isotropic hardening rule. The plastic strain hardening of Al matrix was modeled using a Swift hardening law:

$$
\bar{\sigma}=K\left(\bar{\varepsilon}+\bar{\varepsilon}_{0}\right)^{n}
$$

(Equation 9)

where $K$ is material constant, $\bar{\varepsilon}_{0}$ is a prestrain-like material constant and $n$ is strain hardening exponent. The constants are selected such that the continuum stress-strain curve matches the SD-predicted curve, Figure 15 (a).

11 The experimentally determined contribution of grain size to yield stress of pure aluminum (99.999\%) with a grain size of $1 \mathrm{~mm}$ (same for the Al-1\%Ge-Si alloy) is only $7 \mathrm{MPa}$ [89].

12 In similar simulations for steel alloys [111], it was shown that the choice of boundary conditions is not critical. Simulated stresses throughout tensile and CT tests differed less than $1 \%$ between symmetry and periodic boundary conditions.

13 In fact, while discrete-dislocation interactions are reflected in the formulation, they are implemented in terms of lumped "Superdislocations" (hence the name of the technique) at the center of each finite element. 
$\boldsymbol{C P}$ and $\boldsymbol{S D}$ models - The SD model is a modification ${ }^{14}$ of standard CP models widely used for modeling of textured polycrystals. A summary of the SD model and its implementation here is presented in summary form in the Appendix B to this paper; for full information, the original references are recommended [100,105,109-111].

The original SD model [105] was constructed for treating the elastic interaction of discrete dislocations with each other and with grain boundaries as obstacles at the intersection of slip planes and grain boundaries. Later versions successfully incorporated other dislocation slip obstacles, such as large martensite islands in dual-phase steels [111], where the interaction is primarily at a macro/continuum/composite scale. In the current work, the SD model is further extended to fine-scale dilute $\mathrm{Al}$ alloys, where the precipitates have such a small volume fraction as to be inconsequential in a composite/continuum sense; only the Orowan interactions are significant.

An important aspect of the SD model (as well as the CP model as implemented here) is that the constitutive parameters, with one exception, are determined from single-crystal properties. That is, this is what was called in the Introduction as a new-RVE approach. In this way, the resulting models are predictive such that the predictions can be usefully compared with experiments to evaluate the fidelity of the constitutive approach - and, therefore, the source of the physical phenomenon. However, the one exception is the strength of the alloy, which for accuracy must be used to determine one of the single-crystal properties. This is necessary because usually the dislocation densities at various aging conditions are not well known. Thus, one strength parameter (usually the flow stress of the matrix) must be set using a single stress-strain point from a standard macroscopic tensile test result. Therefore, predicting the Bauschinger effect uses no fit parameters from the $\mathrm{CT}$ test itself. The two results are independent.

The SD (and CP) model discretizes a polycrystal into finite elements, many elements per grain. The equilibrium boundary value problem is solved in the usual way in Abaqus/Standard with the choice of constitutive models being implemented through user subroutines. The solution of the equilibrium equation is implicit, but the coupling at each time step with the user subroutines is explicit. That is, there is no iteration between Abaqus and subroutines at each time step, but there are iterations at each time step within Abaqus and within each of the subroutines.

The SD model consists of two such subroutines:

a) a nearly-standard CP model implemented in Abaqus/Standard, and,

b) a special meso-scale subroutine that computes the local backstress among dislocation populations from element to element and updates dislocation densities on each slip system in each element (along with corresponding local slip resistances) based on Orowan's equation.

\footnotetext{
14 The version used here is presented as a subset of the SD model, see Appendix B.
} 
The only difference in the SD and CP implementations here is the use of the "b" subroutine for SD, not for CP. Therefore, any differences in the predictions between SD and $\mathrm{CP}$ can be attributed to discrete dislocation-dislocation elastic interactions and dislocation-obstacle (precipitate or grain boundary) interactions.

\subsection{Simulation Results and Comparison with Experiment}

Typical simulation results for the PA condition are shown in Figures 15, with Figure 15 (a) presenting the stress-strain results as measured, and Figure 15 (b) presenting the same information in reduced form (like for Figures 13, already introduced) for better resolution of the comparison. The SD model predicts the form and even most of the quantitative details accurately; the Continuum and CP models show virtually no Bauschinger effect. This simple comparison demonstrates that the experimentally-verified linkage of the Bauschinger effect to non-shearable precipitates is a result of dislocation-dislocation elastic interactions, as well as, of course, the obstacle nature of the precipitates to dislocation slip on intersecting slip planes. When the piled-up nature of the discrete dislocation interactions is ignored in the $\mathrm{CP}$ and Continuum models, the Bauschinger effect all-but disappears.

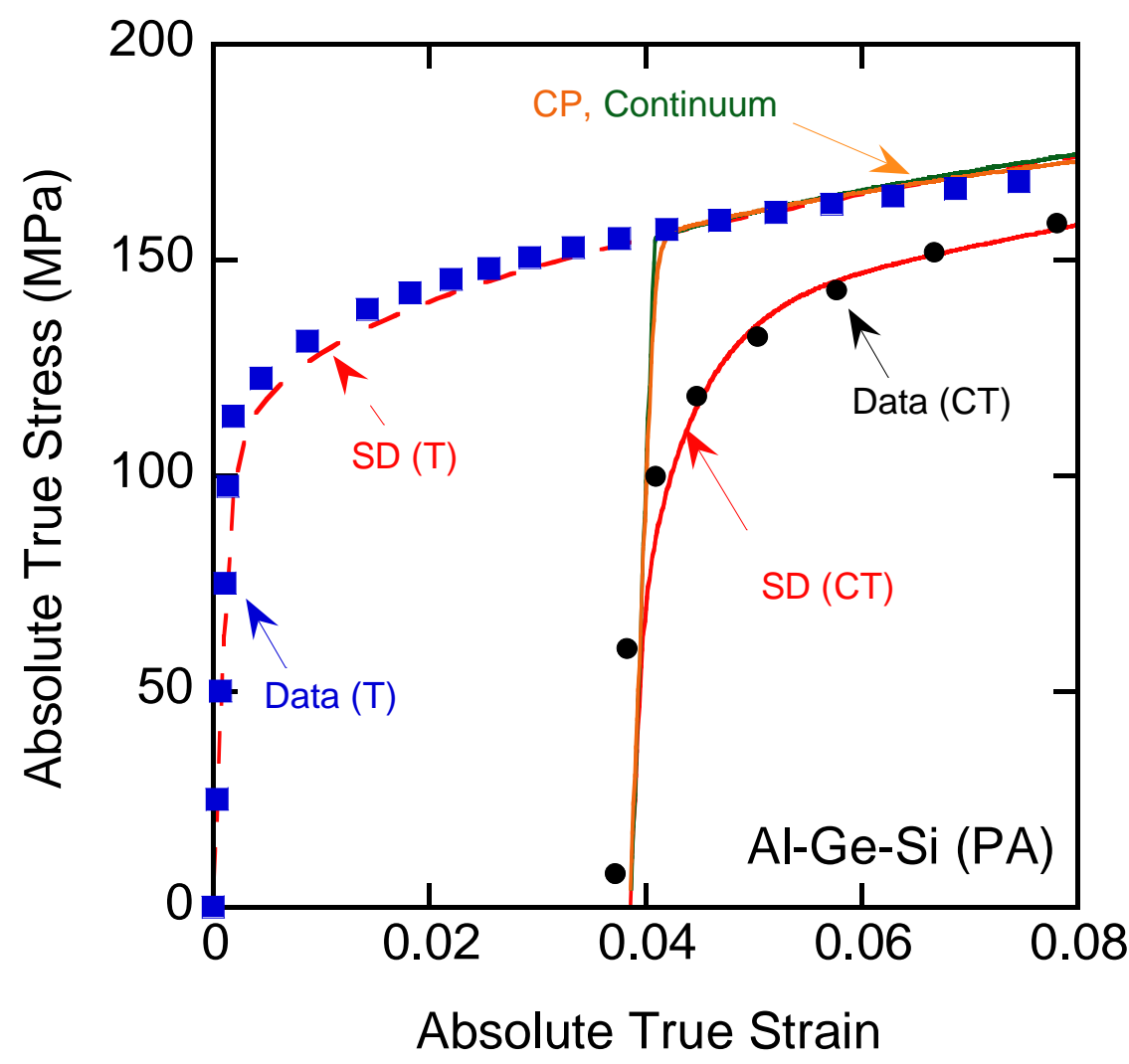

(a) 


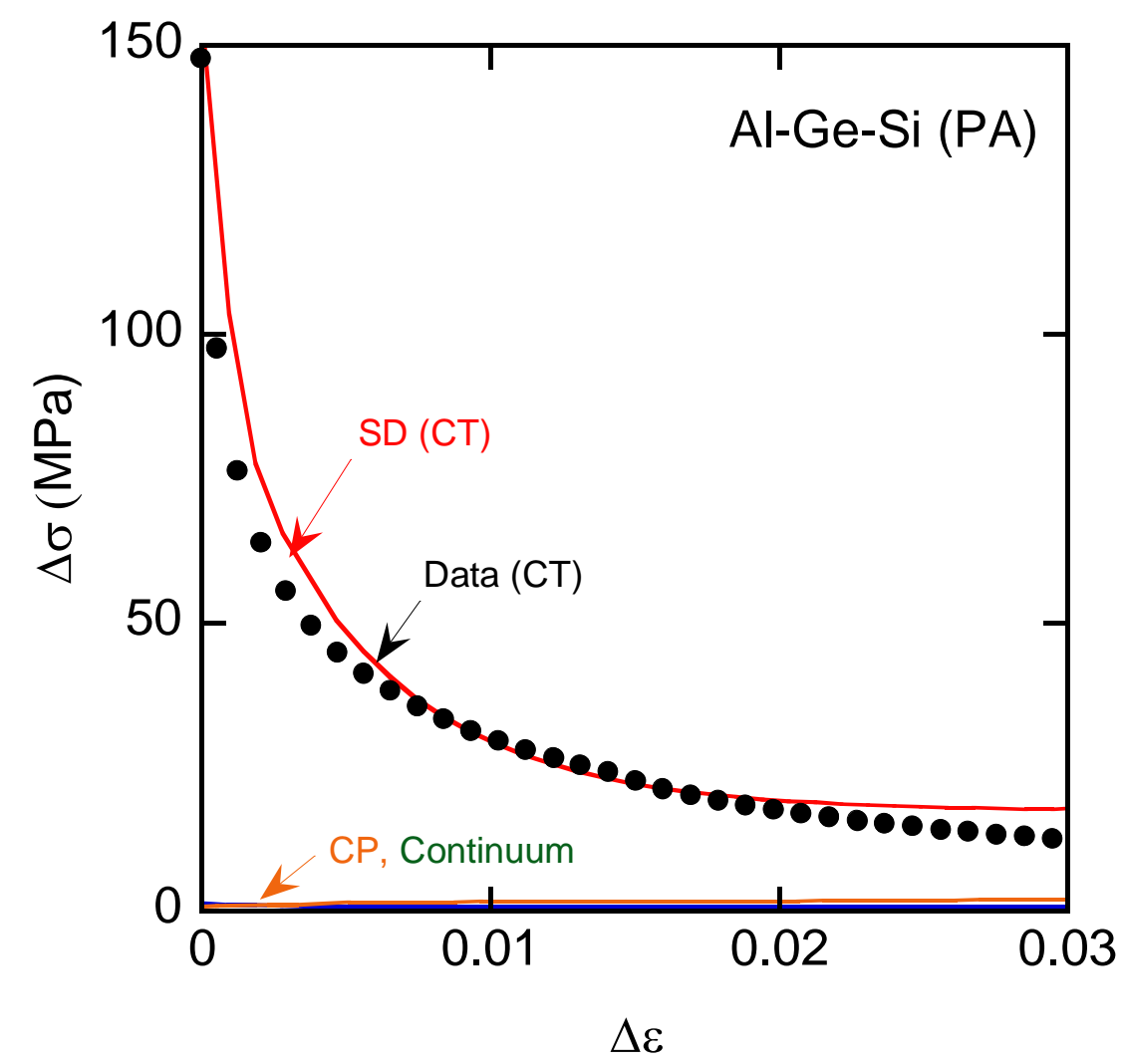

(b)

Figure 15. Comparison of CT transient hardening, experimental and simulated (3 models): (a) stress-strain, and (b) Reduced $\Delta \sigma$ vs. $\Delta \varepsilon$ form.

Figures 16 and Table 5 summarize the full simulation and aging results in terms of the Bauschinger Factors introduced above. The results are consistent across the various heat-treatment conditions in terms of form and general magnitudes. The predicted Bauschinger effect peaks at the PA condition and drops off only slowly thereafter, consistent with the experiments. However, the one notable difference in these aging comparisons is that simulations show substantive symmetry in Bauschinger Factors when moving away from the PA condition toward UA and OA whereas the experiments show much slower, smaller changes for OA conditions than for UA conditions.

Figures 16 and Table 5 also include Bauschinger effect predictions for precipitate-free SHT and pure Al conditions. By comparison with the aged materials, the effect is minimal. It corresponds to the small effect from grain boundaries alone. The UA condition is closer to SHT in terms of the magnitude of the Bauschinger effect, much smaller than for the similar-strength OA condition. 


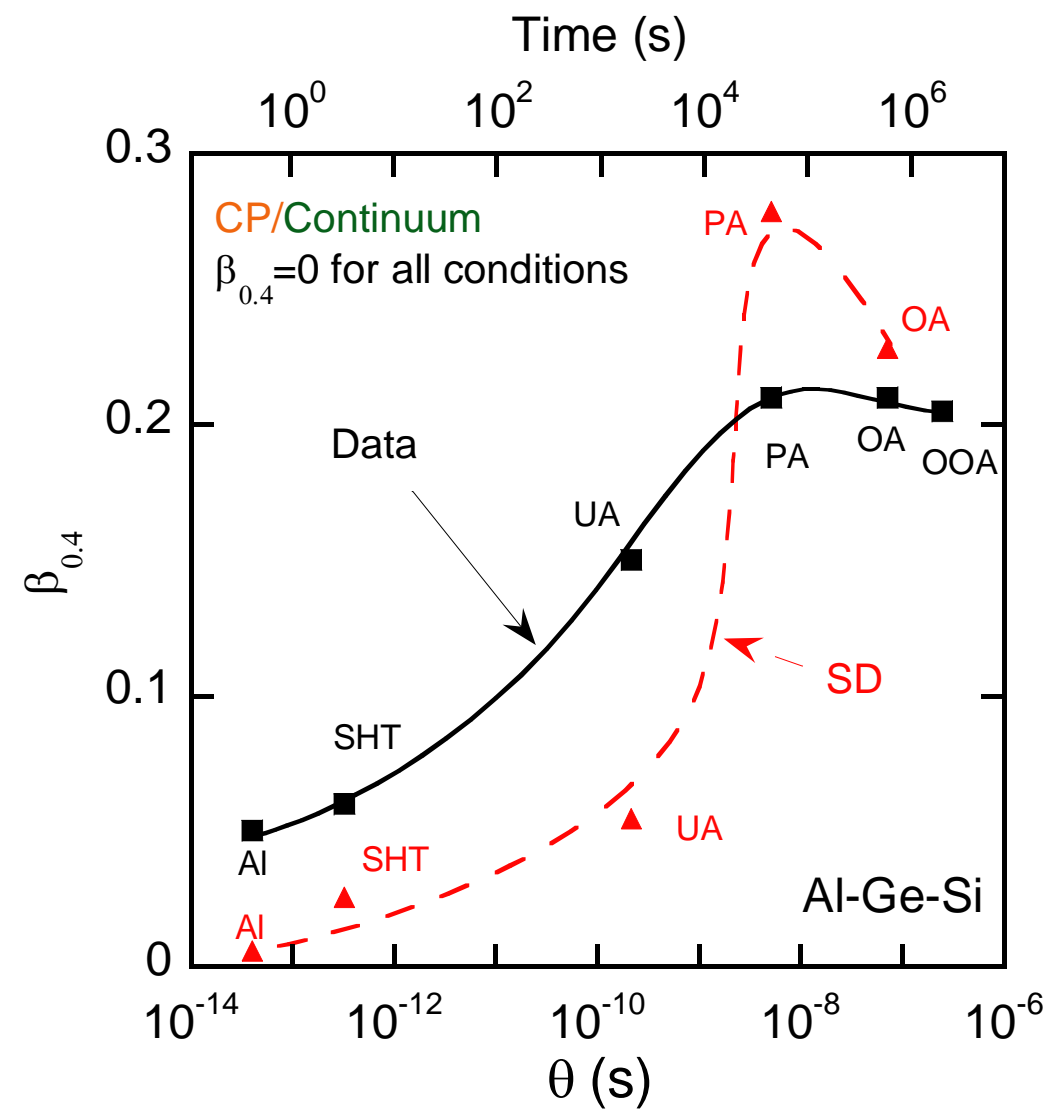

(a) 


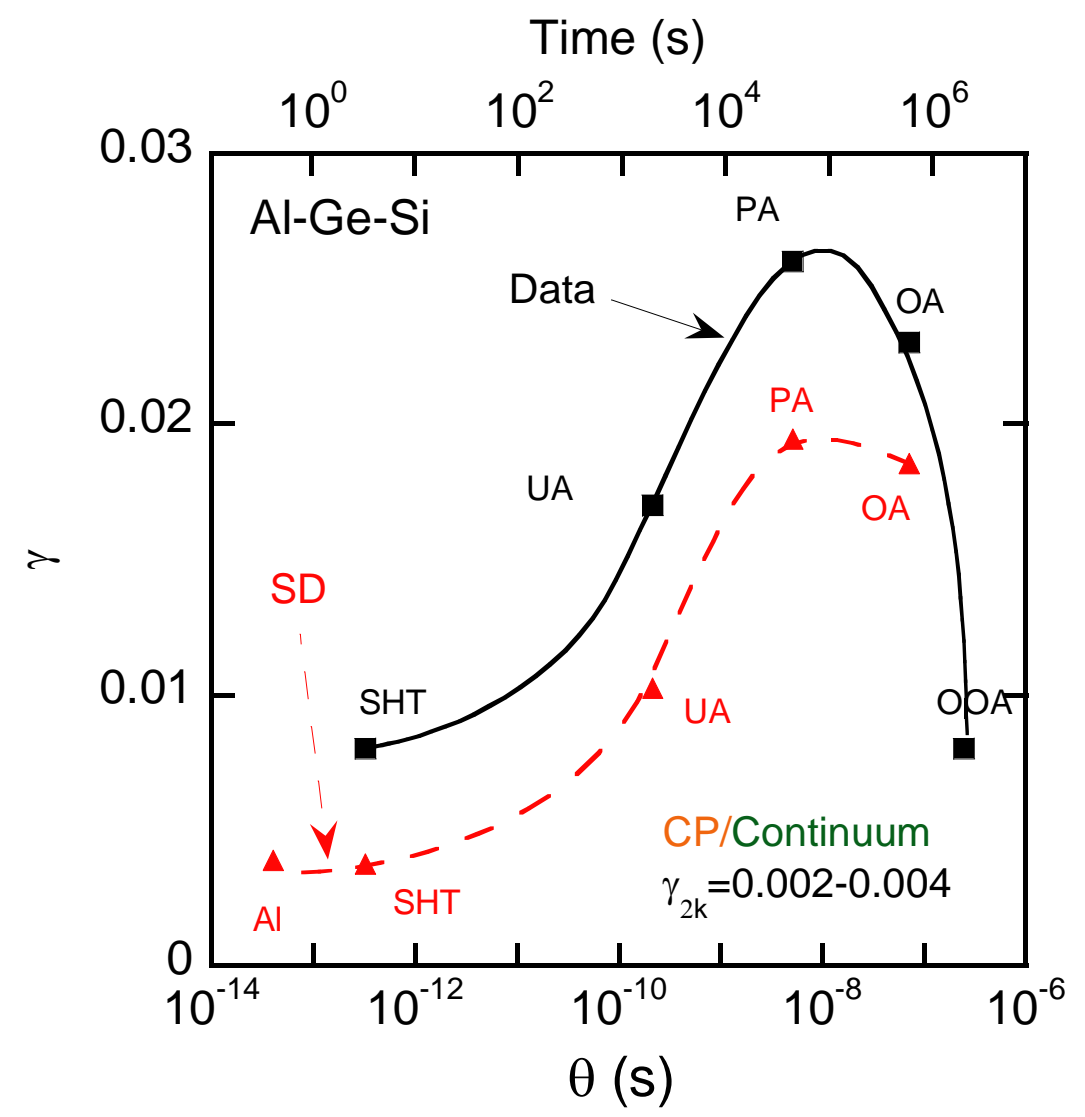

(b) 


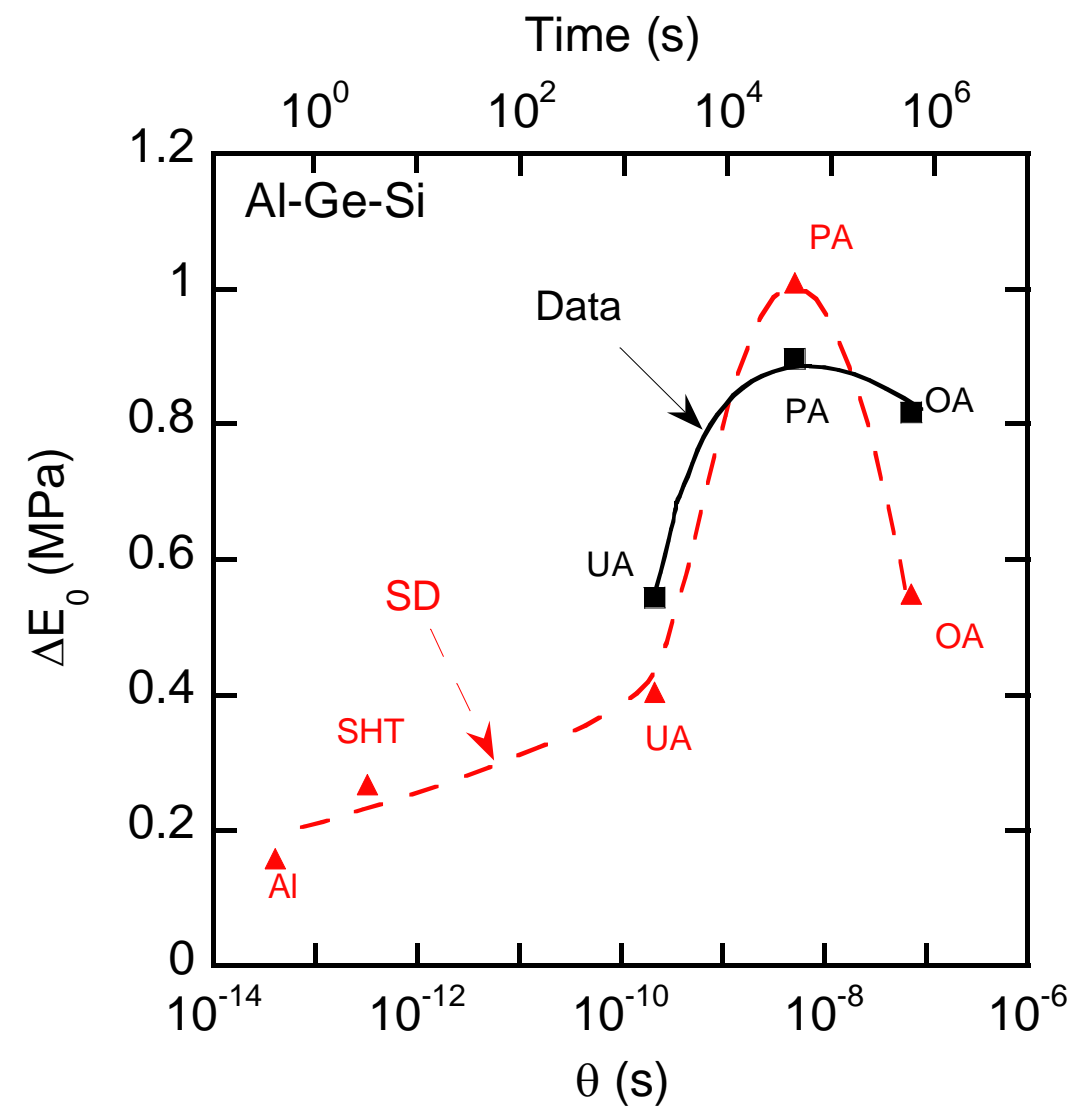

(c)

Figure 16. Comparison of Bauschinger factors from experiments and simulation as a function of aging time: (a) $\beta_{0.4}$ (b) $\gamma_{2 k}$ and (c) $\Delta E_{0}$ (CP and Continuum results are not shown: they like on the abscissa with $\Delta \mathrm{E}=0.00-0.03$. See Table 5)

In view of Figures 16 and Table 5, it is clear that the SD model captures the essence of the strain path reversal and with good quantitative accuracy in each case, while the standard $\mathrm{CP}$ and Continuum simulations predict essentially no Bauschinger effect or transient behavior following the reverse yield. The latter result should have been readily predicable, at least for continuum simulations: such a small volume fraction of hard particles (even infinitely hard, as treated here) cannot have a significant effect on the continuum-predicted hardening. The same turns out to be true even including slip system effects, elastic effects, and other such inhomogeneities. Clearly, the essence of the Bauschinger effect lies with its origin in dislocation-dislocation interactions and dislocation-obstacle interaction, in this case the operant obstacles with precipitates.

Table 5 Predicted Bauschinger variables for aged Al-Ge-Si materials

\begin{tabular}{|l|c|c|c|c|c|c|}
\hline \multicolumn{2}{|c|}{} & \multicolumn{5}{|c|}{ Condition } \\
\cline { 3 - 7 } \multicolumn{2}{|c|}{} & Al & SHT & UA & PA & OA \\
\hline$\beta_{0.4}$ & Experiment & 0.05 & 0.06 & 0.15 & 0.21 & 0.21 \\
\hline
\end{tabular}




\begin{tabular}{|l|c|c|c|c|c|c|}
\hline \multirow{5}{*}{} & SD & 0.01 & 0.03 & 0.05 & 0.28 & 0.23 \\
\cline { 2 - 7 } & Standard CP & 0 & 0 & 0 & 0 & 0 \\
\cline { 2 - 7 } & Continuum & 0 & 0 & 0 & 0 & 0 \\
\hline \multirow{4}{*}{$\gamma$} & Experiment & - & 0.008 & 0.017 & 0.026 & 0.023 \\
\cline { 2 - 7 } & SD & 0.004 & 0.004 & 0.010 & 0.019 & 0.019 \\
\cline { 2 - 7 } & Standard CP & 0.001 & 0.003 & 0.004 & 0.001 & 0.003 \\
\cline { 2 - 7 }$\Delta E_{0}(\mathrm{MPa})$ & Continuum & 0.001 & 0.002 & 0.003 & 0.003 & 0.002 \\
\hline & Experiment & - & - & 0.54 & 0.90 & 0.82 \\
\cline { 2 - 7 } & SD & 0.16 & 0.27 & 0.40 & 1.01 & 0.55 \\
\cline { 2 - 7 } & Standard CP & 0.002 & 0.05 & 0.07 & 0.03 & 0.03 \\
\cline { 2 - 7 } & Continuum & 0.003 & 0.05 & 0.05 & 0.03 & 0.01 \\
\hline
\end{tabular}

\section{CONCLUSIONS}

The aging of Al-Ge-Si alloys and their subsequent mechanical characterization and simulation were used to reveal the role of non-shearable precipitates on mechanical behavior, thus separating out the confounding transition from shearable to non-shearable precipitates in typical Al age-hardening alloys. Two kinds of conclusions were reached, the first with respect to purely microstructural features associated with aging of Al-Ge-Si alloys, and the second with respect to experimental and mechanistic aspects of the reverse yield and hardening (generalized Bauschinger effect).

\section{Aging of Al-Ge-Si}

1. The aging curves of 1 atomic percent Al-Ge-Si at three temperatures fall on a single master curve with little scatter using a reduced temperature-time variable based on published values of the activation energy of diffusion of germanium in aluminum.

2. Consistent with the Orowan equation, the precipitate spacing passes through a minimum at the peak aging condition, as defined by maximum hardness and yield stress. Concurrently, the volume fraction of precipitates increases dramatically to its full value, and it maintains a plateau thereafter, representing a condition with nearly all the solute out of the matrix.

3. While the peak hardness and yield strength occur at the PA condition, and the OA and UA conditions represent equal-but-lower hardness and strength, the strain hardening is almost identical for OA and PA conditions, and the Bauschinger effect is similar.

4. The role of solute content on strain hardening was quantified with an equation for 3 solution-treated Al-Ge-Si alloys. Explicit equations describing strain hardening and dislocation density evolution in terms of solute content were presented. 
5. Consistent with results from the literature, solutes in aluminum greatly increase strain hardening. At sufficiently large strains under some conditions, precipitation effectively softens the alloys.

6. Under most aging conditions tested, the morphology of the Ge-Si precipitates remains nearly equiaxed. The precipitate size increases monotonically with aging, while the size distribution widens.

\section{Bauschinger Effect -Experimental}

7. Bauschinger Factors have been introduced to characterize the nature of reverse hardening in terms of stress, strain and combined stress/strain (energy). These tend to rise and fall together. This result may not be universal [96].

8. Approximately $70 \%$ of the Bauschinger effect for the PA and OA conditions in these very dilute alloys is attributable to precipitates, with the remainder attributable to other obstacles, primarily grain boundaries.

9. The Bauschinger effect is generally maximized when the strength is maximized, i.e., the PA condition. However, it declines only slowly with further aging - the $\mathrm{OA}$ and PA conditions exhibit similar Bauschinger Factors although the precipitate spacing differs by $60 \%$.

10. The Bauschinger effect for $\mathrm{OA}$ is much greater than UA, even though they exhibit similar strength. These observations imply that the Bauschinger effect for the non-shearable precipitates is not related only to simple Orowan effects arising from pin spacing.

\section{Bauschinger Effect - Mechanistic}

11. Simulations show that the dominant mechanism controlling the Bauschinger effect is the generation of internal stresses by polarized dislocations that are "piled-up" (in a general sense) against obstacles and which interact to create a back stress that either resists strain (forward direction) or assists it (reverse direction). Whether the actual dislocation configuration can be ideally characterized as pile-ups of parallel dislocations, or bow-out of many dislocations between hard pins, or another conceptually simple configuration, cannot be answered by the current work.

12. An RVE with properties determined by single-crystal, precipitate-free behavior and an "SD" constitutive model accounting for elastic dislocation-dislocation, dislocation-precipitate, and dislocation-grain boundary interactions predicted all of the significant features of the observed Bauschinger effect. Such simulations have no artificial for fit length scales; instead they rely on measured microstructural scales and the Burgers vector. Corresponding Continuum and $\mathrm{CP}$ simulations, which have no real length scale, predict essentially no 
Bauschinger effect for these alloys.

13. The reverse yielding and flow behavior of the very dilute Al alloys with non-shearable precipitates cannot be predicted by composite models (Eshelby theory), but is instead attributable to the interaction between precipitates and dislocations.

14. The RVE based FE simulations for pure Al, SHT, PA, UA and OA conditions reveal that the observed Bauschinger behavior is related not only to precipitate spacing, but also to volume fraction and/or particle size. Nonetheless, the observed behavior is reproduced closely by the SD model predictions.

15. In $\mathrm{Al}$ alloys with non-shearable precipitates, even very small volume fractions, the effect of grain boundaries on the Bauschinger effect is minor, contrary to larger effect in ferrous alloys. Nonetheless, for the SHT condition or for pure Al with normal grain sizes, the Bauschinger effect is still well-predicted by the SD model.

\section{ACKNLOWLEDGEMENTS}

This research is supported by the U.S. Department of Energy, Office of Science, Office of Basic Energy Sciences, Materials Science and Engineering Division, and particularly Program Director John S. Vetrano, who are thanked for their support of the current work under Award Number DE-SC0012483 and DE-SC0012587. Thanks also to the Alcoa Technical Center, particularly Robert Hyland (currently at U.S. Steel), for partially funding the original experiments many years ago and for providing the aluminum-germanium-silicon alloys. 


\section{APPENDIX A： Strain Hardening by Solutes}

In order to characterize quantitatively the strain hardening as a function of solute content, the stress-strain curves of two alloys in the SHT condition (i.e. precipitate-free, fully solid solution strengthened) were compared with that of the pure Al (99.999\% purity [89]), Figure A1(a). Note that the solutes dramatically increase the strain hardening rate. The difference between the solute-strengthened strain hardening curves and the pure $\mathrm{Al}$ baseline hardening curve can be attributed to solid solution hardening strengthening; all other microstructural aspects are maintained constant.

This aspect must be taken into account with any predictive simulation of age hardening beyond yield (as for the Bauschinger effect, for example). In essence, at significant strains, the aging drastically reduces the progressive strength of the matrix while the precipitates act as obstacles for Orowan hardening.

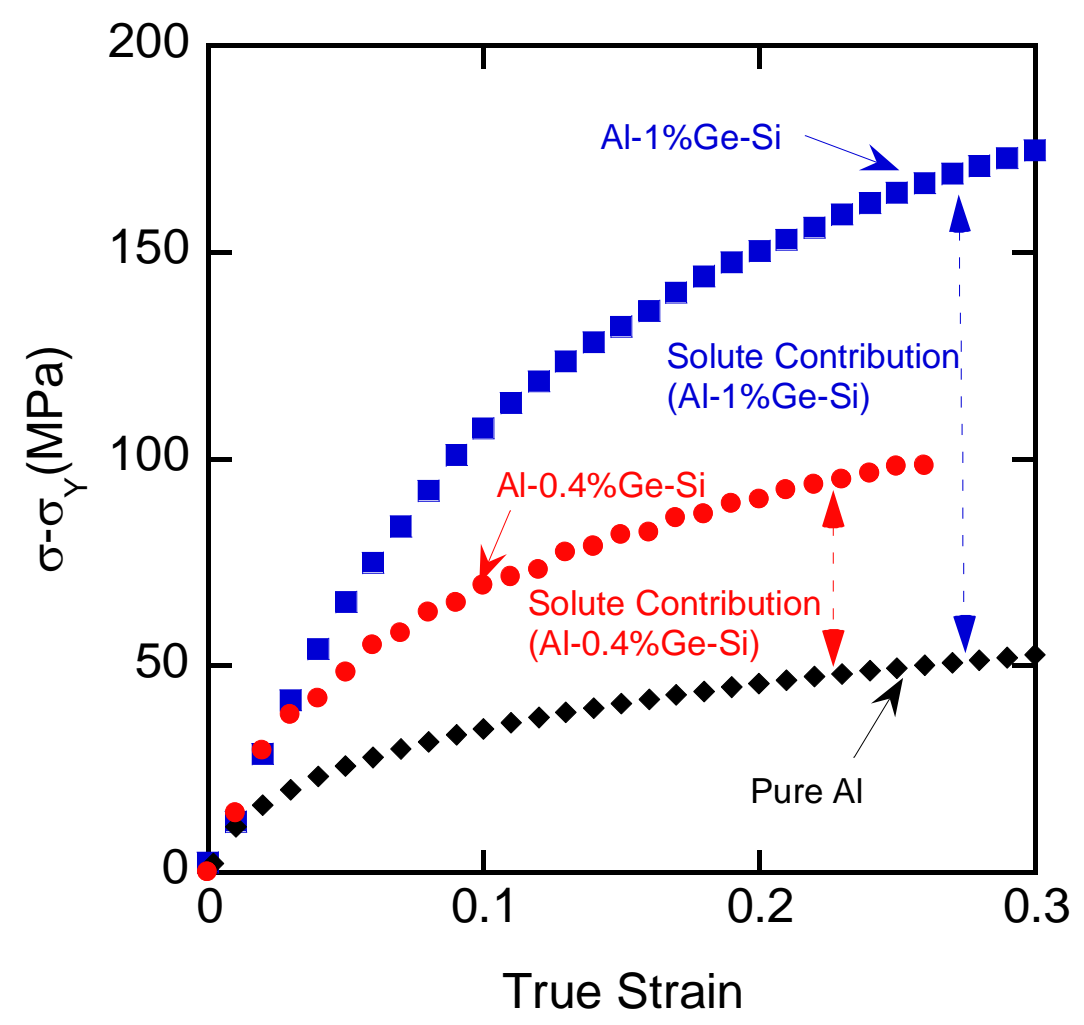

(a) 


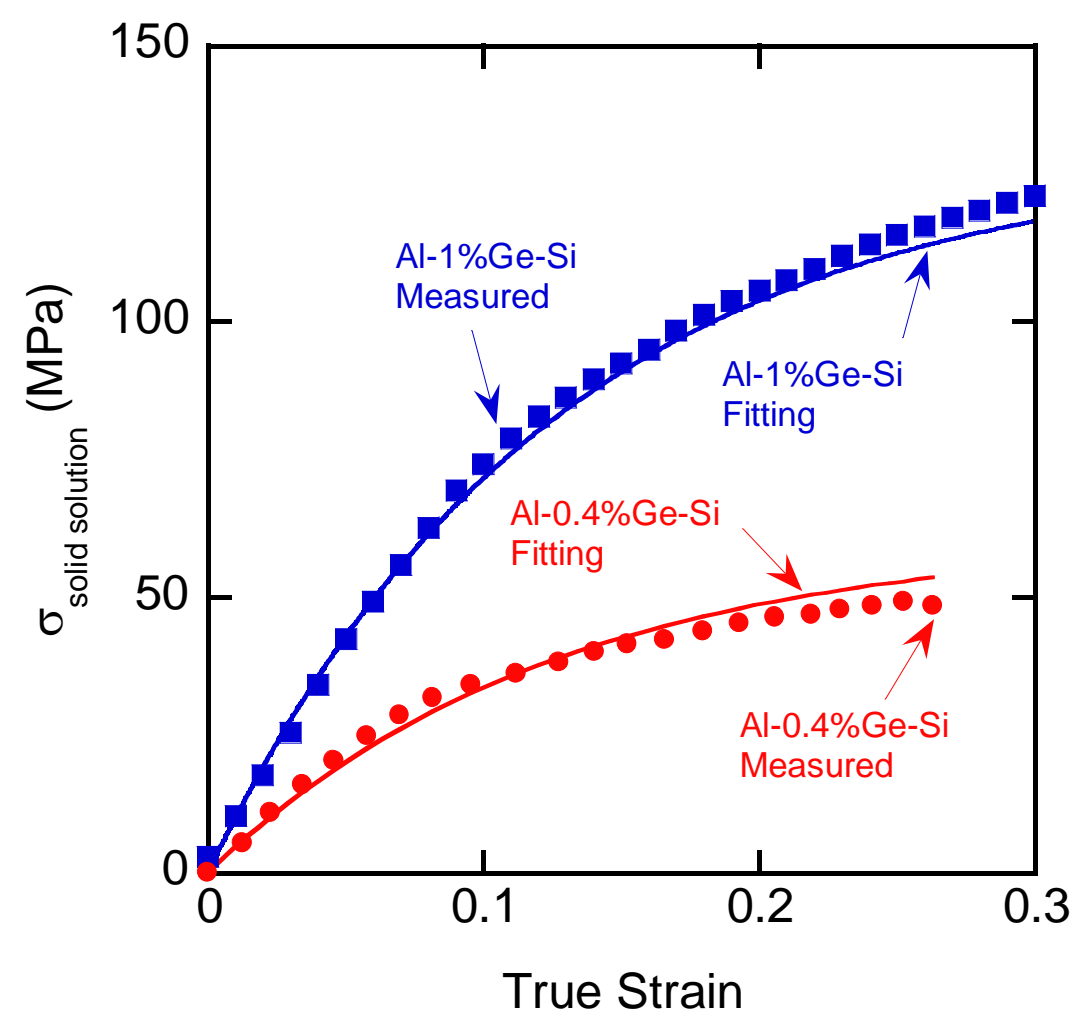

(b)

Figure A1. Role of solutes on strain hardening after yielding: (a) measured results for two alloys with different solute concentration, and (b) reproduction of these results with a novel modified-Voce equation.

Motivated by the form of Equation 7 describing the variation of yield stress with respect to solute concentration, the full Voce-type strain hardening for a range of solute-strengthened Al-Ge-Si alloys can be expressed similarly, as follows:

$$
\sigma_{\mathrm{ss}}=\{\mathrm{a}-\mathrm{b} \times \exp (-\mathrm{c} \varepsilon)\} \mathrm{C}^{2 / 3}
$$

(Equation A1)

where $\mathrm{a}, \mathrm{b}$ and $\mathrm{c}$ are typical Voce strain hardening constants ${ }^{15}$ and $\mathrm{C}$ is again the solute concentration expressed in (atomic percent) ${ }^{2 / 3}$. The explicit equation found is as follows:

$$
\sigma_{\mathrm{ss}}=\{2.6 \mathrm{GPa}-2.6 \mathrm{Gpa} \times \exp (-8 \varepsilon)\} \mathrm{C}^{2 / 3}
$$

15 The constants a and $\mathrm{b}$ are identic because $\sigma_{\mathrm{ss}}$ is identically 0 at 0 strain. 
The solid solution hardening curves for both Al-1\% Ge-Si and Al-0.4\% Ge-Si materials are shown in Figure A1(b).

\section{APPENDIX B: SD and CP Constitutive Models and Implementation}

The development below is intended to provide sufficient background to understand the constitutive models and their implementation in the current work. For more detail, please refer to the original papers [100,105,109-111].

\section{SD and CP Models}

The SD and CP models are similar, except for the former uniquely accounting to dislocation-dislocation interactions and dislocation-obstacle interaction. In both models, a classical crystal plasticity framework is employed [100,112-114]. The viscoplastic shear rate in power-law form was represent the shear rate on the $\alpha$ slip system, $\dot{\gamma}^{(\alpha)}$, as follow [113]:

$$
\dot{\gamma}^{(\alpha)}=\dot{\gamma}_{0}\left(\frac{\tau^{(\alpha)}}{g^{(\alpha)}}\right)^{1 / m} \operatorname{sign}\left(\tau^{(\alpha)}\right)
$$

where $\tau^{(\alpha)}$ is the resolved shear stress on the slip system $\alpha, \dot{\gamma}_{0}$ is a reference shear rate, $m$ is a strain rate sensitivity ${ }^{16}$, and $g^{(\alpha)}$ is the slip resistance on the slip system $\alpha$.

In both $\mathrm{CP}$ and $\mathrm{SD}$ implementations, a dislocation density-based constitutive equation for single crystals is adopted [109]. The initial dislocation density $g_{0}$ is equally partitioned onto all slip systems in each element. The slip resistance of slip system $\alpha, g^{(\alpha)}$, evolves with plastic shear strain on all co-located slip systems according to

$$
g^{(\alpha)}=A \mu b \sqrt{\sum_{\beta=1}^{N S} \boldsymbol{h}_{\alpha \beta} \rho^{(\beta)}}
$$

where $\mu$ is the shear modulus, $b$ is the Burgers vector, $\rho^{(\alpha)}$ is the dislocation density in slip system $\alpha, \boldsymbol{h}_{\alpha \beta}=\boldsymbol{n}^{(\alpha)} \boldsymbol{\xi}^{(\beta)}$ represents the interaction cosines where $\boldsymbol{n}^{(\alpha)}$ and $\boldsymbol{\xi}^{(\beta)}$ are the slip plane normal of slip system $\alpha$ and the dislocation line vector of

16 The parameter $m$ is not strictly a physical strain-rate sensitivity. It has no connection to real time. Rather, it is a mathematical convenience to simplify the apportionment of strain onto various competing slip systems. This is a characteristic of the standard visco-plastic formulations for polycrystals in common use. 
slip system $\beta$, respectively. The details of geometric formulation are given elsewhere [109]. The parameter $A$ is a material constant that ranges from 0.3-0.6 [115,116]. A value of 0.4 is assumed in the SD and CP models.

The dislocation density evolves according to a well-known dislocation density evolution equation [117]:

$$
\rho^{(\alpha)}=\frac{1}{b}\left(\frac{\sqrt{\sum_{\beta}^{N S} \rho^{(\beta)}}}{k_{a}}-k_{b} \rho^{(\alpha)}\right) \gamma^{(\alpha)}
$$

where $k_{a}$ and $k_{b}$ are material parameters conceptually related to dislocation generation and annihilation, respectively, but are typically determined from strain hardening experiments. The phenomenological nature of Equation B3 has not proven amenable to microstructurally-based prediction or simulation, thus reference to at least some stress-strain data is required in any implementation involving large-strain deformation.

The foregoing describes the entirety of the $\mathrm{CP}$ constitutive model, and part of the SD model. The remainder of this section deals with the additional aspects of the SD model to account for dislocation-dislocation and dislocation-obstacle interactions.

In the SD treatment, the dislocation density for each slip system in each element is treated as a single super-dislocation located at the centroid of the element. Mobile dislocation content at the end of each time step is calculated to accommodate the strain gradient and is then redistributed according to the Orowan equation [118] in the view of the shear strain increment throughout the body:

$$
\dot{\rho}_{p a s s}^{(\alpha)}=\frac{1}{b l^{(\alpha)}} \dot{\gamma}^{(\alpha)}
$$

where $\dot{\rho}_{\text {pass }}^{(\alpha)}$ is the rate of dislocation density passing through the element, $l^{(\alpha)}$ is the length of the element parallel to the slip plane and $\dot{\gamma}^{(\alpha)}$ is the shear rate.

The redistributed dislocation densities, in the form of the magnitude of the superdislocations on each slip system in each element are used to calculate the elastic interaction forces among them using analytical solutions for the anisotropic elastic fields of parallel dislocation segments [105,119]. Each such force is introduced as a backstress encountered on each slip system in each element, $\tau_{b}^{(\alpha)}$, into suitably modified Equation B1 as follows:

$$
\dot{\gamma}^{(\alpha)}=\dot{\gamma}_{0}\left(\frac{\tau_{e f f}^{(\alpha)}}{g^{(\alpha)}}\right)^{1 / m} \operatorname{sign}\left(\tau_{e f f}^{(\alpha)}\right)
$$


where $\tau_{\text {eff }}^{(\alpha)}=\tau^{(\alpha)}-\tau_{b}^{(\alpha)}$.

For interactions with grain boundaries, the SD subroutine enforces a critical local stress for passage of slip through a grain boundary [105] or phase boundary as follows:

$$
\tau_{o b s}=(1-N) \tau^{*}
$$

where $\tau_{o b s}$ is the obstacle strength when the slip across a grain boundary occurs, $\tau^{*}$ is a generally-unknown maximum obstacle strength. There is very little data useful for estimating the value of $\tau^{*}$, which in all SD predictions to date is taken to be 5 times of macroscopic yield stress, based on TEM measurements from the 1980's [120]. $N$ as implemented here is a geometrical transmissivity factor determined from the the SWC $2^{\text {nd }}$ Criterion (Shen, Wagoner, Clark [120]) as follows:

$$
N=\left(L_{1} \cdot L_{i}\right) \times\left(g_{1} \cdot g_{i}\right)
$$

where $L_{1}$ and $L_{i}$ are the intersection line vectors between grain boundary and slip planes, and $g_{1}$ and $g_{i}$ are the slip direction vectors of incoming and transmitted dislocations, respectively. Other estimates of the obstacle stress $\tau_{o b s}$ can be formulated and implemented, but verifying any such formulation has proven challenging.

The obstacle strength was incorporated for grain boundary elements by following equations:

$$
\begin{array}{cc}
\tau_{e f f}^{(\alpha)}>\tau_{o b s}^{(\alpha)} & \dot{\gamma}^{(\alpha)}=\dot{\gamma}_{0}\left(\frac{\tau_{e f f}^{(\alpha)}-\tau_{o b s}^{(\alpha)}}{g^{(\alpha)}}\right)^{1 / m} \operatorname{sign}\left(\tau_{e f f}^{(\alpha)}-\tau_{o b s}^{(\alpha)}\right), \\
\tau_{e f f}^{(\alpha)} \leq \tau_{o b s}^{(\alpha)} & \dot{\gamma}^{(\alpha)}=0
\end{array}
$$

(Equation B8)

Equations B6-B9 were also applied to phase boundaries in the current work, i.e. between the $\mathrm{Al}$ matrix and $\mathrm{Ge} / \mathrm{Si}$ precipitates.

\section{Parameter Identification}

In principle, the constitutive parameters $k_{a}, k_{b}, \rho_{0}$ must be determined for each method (SD or CP) and for each microstructural phase (Al or GeSi precipitates) in each heat-treatment condition. In practice, the precipitates are nearly rigid so only the $\mathrm{Al}$ with and without $\mathrm{Ge}$ and $\mathrm{Si}$ solutes needs careful characterization. If parametric values are set from macroscopic experimental data (the typical method appearing in the literature for $\mathrm{CP}$ ), they can vary widely between SD and CP models because of the strong effect of elastic dislocation interactions in SD that are ignored, and thus incorporated obscurely, in the standard CP approach. 
Two methods were used to determine the required parametric values. The primary (and purer) one relies on measurements for single-crystals of pure aluminum in the literature. This method, called the "Micro Approach" here, allows for independent predictions of macroscopic behavior using only micro measurements for the constituent phases. All of the aging conditions were simulated with this approach.

The second approach, the "Macro Approach," adopts the more common (and less revealing) method of back-fitting model parameters from the mechanical behavior of macro polycrystalline solids. This was done only for the PA condition in order to probe whether the difference would be relevant to the critical Baushinger effect predictions.

In both approaches, anisotropic elasticity constants of $\mathrm{C}_{11}=107 \mathrm{GPa}, \mathrm{C}_{12}=61 \mathrm{GPa}$ and $\mathrm{C}_{44}=28 \mathrm{GPa}$ were adopted from the literature for $\mathrm{Al}$ [100], and the same values were used for the $\mathrm{Ge}-\mathrm{Si}$ precipitates, although the orientation of the precipitates was varied to mimic various incoherent orientations. In both methods $\rho_{0}^{G e-S i}$ was set to be 10 times higher than in $\mathrm{Al}^{17}$ to simulate an elastic-only precipitate properties in order to avoid yielding, thus making them plastically rigid.

For all SD simulations, the transmissivity factor, $N$, to calculate $\tau_{o b s}$ was set to 0 to block dislocation transmission from precipitates to matrix, consistent with the rigid-precipitate assumption. $\tau^{*}$ was set for all cases at 5 times of measured macroscopic yield stress following earlier developments ${ }^{18}$ [105,110], e.g., $575 \mathrm{MPa}$ for the PA condition.

\section{SD model (Micro Approach)}

For the Micro Approach, $k_{a}$, and $k_{b}$ for the pure Al phase were obtained such that a single-crystal SD simulation reproduced the measured stress-strain response of a $<100>$-oriented tensile specimen of single crystal, pure Al [121]. The $\rho_{0}$ value was determined from the SD-simulated (SD using the already-determined ( $k_{a}$, and $k_{b}$ ) yield stress for a pure Al polycrystal reported in the literature [89]).

For the matrix phase for all but the pure aluminum, Equation 8 was used in conjunction with an SD simulation of a pure aluminum polycrystal to obtain a predicted tensile-stress-strain curve for each matrix with known solute content. That is, a stress-strain curve for pure polycrystal aluminum was simulated and the $\sigma_{\text {ss }}$ determined from Equation 8 for the particular condition (SHT, UA, PA, or OA) was added to it. Then,

17 The assumed initial dislocation density in Ge/Si particles imposes $250 \mathrm{MPa}$ yield strength increase, higher than the ultimate tensile strength of the alloy. Other simulations were conducted with $10^{2}$ times higher, corresponding to $\sim 800 \mathrm{GPa}$ yield strength increase. No difference in overall stress-strain or Bauschinger behavior was predicted.

18 The assumed value (5 times of macroscopic yield stress) was actually assumed for grain-to-grain interfaces in the earlier works, but same assumption was employed for grain-to-particle interface in this study. 
the polycrystalline total stress-strain curve for the matrix was used as for the case of pure aluminum to determine $\mathrm{k}_{\mathrm{a}}$ and $\mathrm{k}_{\mathrm{b}}$ for that matrix. The $\rho_{0}$ values for these cases was determined as for the pure aluminum, the only difference being that the appropriate $\mathrm{k}_{\mathrm{a}}$ and $\mathrm{k}_{\mathrm{b}}$ values for the aging condition were used in the particular simulation, as was the measured yield stress for the macro specimen.

The $k_{a}{ }^{A l}$ and $k_{b}{ }^{A l}$ values determined as described above for the matrices of the various aging conditions for the Al-1\% Ge-Si alloy, and pure aluminum, are shown in Figure B1, along with lines corresponding to equations generated using Equation 8 and the procedure described above.

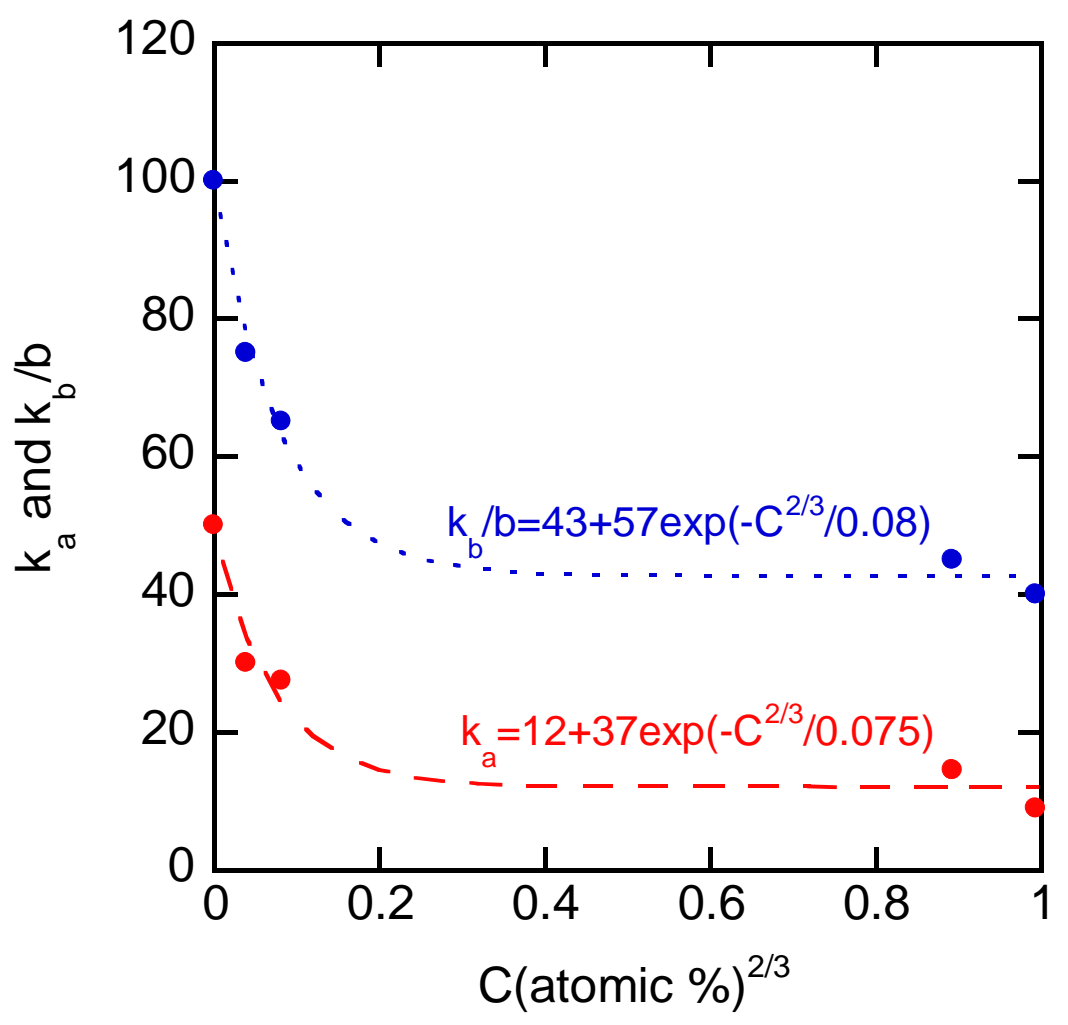

Figure B1. Variation of $\mathrm{k}_{\mathrm{a}}$ and $\mathrm{k}_{\mathrm{b}}$ as a function of the concentration of solutes. Points represent results for 3 tested $\mathrm{Al}-\mathrm{Ge}-\mathrm{Si}$ alloys, lines represent derivation from Equation 8 $\left(b=2.87 \times 10^{-10} \mathrm{~m}\right)$.

Simulations using the resulting Micro constitutive model are compared with CT experiments in Figure B2. Note that, as expected, the tensile strain hardening is not predicted perfectly by the micro approach. This is because of the phenomenological nature of strain-hardening and the only fitting of model parameters being to a single quantity, the yield stress, obtained from macroscopic experiments. 
Surprisingly, the difference in strain hardening is insignificant in terms of the main predictions: i.e. the Bauschinger effect, as is shown in Figure 15. (The prediction of delta sigma vs delta strain is independent of the choice of the Micro or Macro methods.) Nonetheless, the difference in strain hardening obscures the accuracy of the prediction when only raw stress-strain data is plotted, as it is in Figure B2. In order to simplify the appearance of the predictions in terms of raw stress and strain, an alternate Macro approach to parameter identification was used in an attempt to better reproduce the strain hardening observed in the macro specimens.

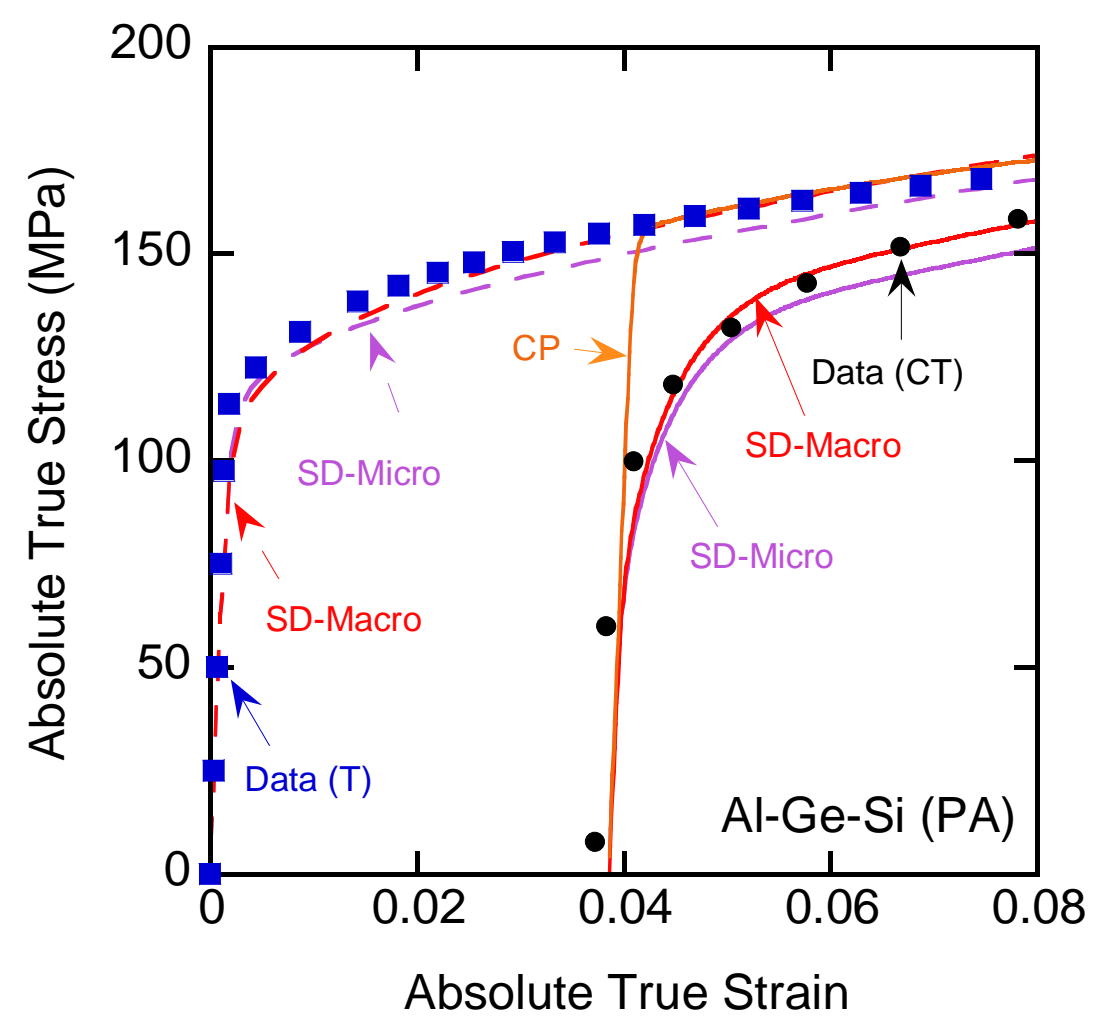

Figure B2. Comparison of simulated and experimental tensile (T) and compression-tension (CT) tests. The first, tensile, leg of the CT experiment is identical to the tensile and is not shown to promote clarity.

\section{SD model (Macro Approach)}

In the macro approach to parameter identification, $k_{a}{ }^{A l}$ and $k_{b}{ }^{A l}$, were fit directly to the measured macro stress-strain curve for the polycrystalline alloy instead of using single-crystal data from the literature. Thus, in addition to fitting the overall stress level to the macroscopic tensile result (as in the Micro case), the strain hardening parameters are also fit to the macroscopic tensile result. The procedure makes no difference for the prediction of the critical Bauschinger effect, but makes it easier to see the agreement in terms of raw stress-strain data from the CT test, Figure B2 (compare purple line and red line, both of which correspond to Figure 15). Note that in both the Macro and Micro 
approaches, nothing measured in the CT tests was used in constructing the constitutive equations, only monotonic tensile test results.

\section{CP Model (Macro Approach)}

Using standard methods for fitting CP model parameters, $k_{a}{ }^{i}, k_{b}{ }^{i}, \rho_{0}{ }^{i}$ were optimized to reproduce the tensile test stress-strain curves for each aging condition. This is the standard procedure followed in the literature: it corresponds to the Macro approach described for the SD Model. As for the SD Model, nothing was used from CT test results. All the other parameters were set identically to the SD models. The final parameters are shown in Table A1.

Table A1. Constitutive parameters for the three constitutive models

\begin{tabular}{|c|c|c|c|c|c|}
\hline & $\begin{array}{l}\text { Constitutive } \\
\text { Model }\end{array}$ & $\begin{array}{c}k_{a}^{A l} \\
\left(=k_{a}^{G e-S i}\right)\end{array}$ & $\begin{array}{c}k_{b}{ }^{A l} \\
\left(=k_{b}{ }^{G e-S i}\right) \\
(*)\end{array}$ & $\begin{array}{c}\rho_{0}{ }^{A l} \\
\left.\rho_{0}{ }^{G e-S i} / 10^{5}\right) \\
\left(/ \mathrm{m}^{2}\right)\end{array}$ & $\begin{array}{c}\tau^{*} \\
(\mathrm{MPa})\end{array}$ \\
\hline \multirow[t]{2}{*}{$\mathrm{Al}$} & $\mathrm{CP}$ & 38 & $125 \mathrm{~b}$ & $1.0 \times 10^{12}$ & - \\
\hline & SD-Micro & 50 & $100 \mathrm{~b}$ & $1.0 \times 10^{12}$ & 35 \\
\hline \multirow[t]{2}{*}{ SHT } & $\mathrm{CP}$ & 9.5 & $53 b$ & $0.5 \times 10^{14}$ & - \\
\hline & SD-Micro & 9 & $40 \mathrm{~b}$ & $0.5 \times 10^{14}$ & 235 \\
\hline \multirow[t]{2}{*}{ UA } & $\mathrm{CP}$ & 8.5 & $77 b$ & $2.9 \times 10^{14}$ & - \\
\hline & SD-Micro & 14.5 & $45 b$ & $2.8 \times 10^{14}$ & 468 \\
\hline \multirow[t]{3}{*}{$\mathrm{PA}$} & $\mathrm{CP}$ & 8 & $101 b$ & $4.5 \times 10^{14}$ & - \\
\hline & SD-Micro & 27.5 & $65 b$ & $3.5 \times 10^{14}$ & 573 \\
\hline & SD-Macro & 5 & $320 b$ & $3.5 \times 10^{14}$ & 573 \\
\hline \multirow[t]{2}{*}{$\mathrm{OA}$} & $\mathrm{CP}$ & 8 & $135 b$ & $3.1 \times 10^{14}$ & - \\
\hline & SD-Micro & 30 & $75 b$ & $2.8 \times 10^{14}$ & 482 \\
\hline
\end{tabular}

(*) $b=2.87 \times 10^{-10} \mathrm{~m}$

\section{ACKNOWLEDGEMENTS}

This research was supported by the U.S. Department of Energy (DOE), Office of Science, Office of Basic Energy Sciences, Materials Science and Engineering Division, and particularly program director John S. Vetrano, for their support of the current work under Award Number DE-SC0012483 and DE-SC0012587. 


\section{REFERENCES}

[1] J. Bauschinger, Tech. Hochschule Munchen, H. 13 (Abschnitt 5) (1886) 31.

[2] L. Geng, Y. Shen, R.H. Wagoner, Int. J. Plast. 18 (2002) 743-767.

[3] L. Geng, R.H. Wagoner, Int. J. Mech. Sci. 44 (2002) 123-148.

[4] J. Lee, J.Y. Lee, F. Barlat, R.H. Wagoner, K. Chung, M.-G. Lee, Int. J. Plast. 45 (2013) 140-159.

[5] J.-Y. Lee, J.-W. Lee, M.-G. Lee, F. Barlat, Int. J. Solids Struct. 49 (2012) $3562-3572$.

[6] R.H. Wagoner, H. Lim, M.-G. Lee, Int. J. Plast. 45 (2013) 3-20.

[7] R.H. Wagoner, J.F. Wang, M. Li, in:, ASM Handb., Materials Park, OH, 2006, pp. $733-755$.

[8] D. Raabe, F. Roters, F. Barlat, L.-Q. Chen, Continuum Scale Simulation of Engineering Materials, Fundamentals - Microstructures - Process Applications, Wiley-VCH, Weinheim, 2004.

[9] W.D. Carden, L.M. Geng, D.K. Matlock, R.H. Wagoner, Int. J. Mech. Sci. 44 (2002) 79-101.

[10] K.P. Li, W.P. Carden, R.H. Wagoner, Int. J. Mech. Sci. 44 (2002) 103-122.

[11] A.B. Doucet, R.H. Wagoner, Metall. Trans. A 20 (1989) 1483-1493.

[12] J. V Laukonis, R.H. Wagoner, Metall. Trans. A 16 (1985) 421-425.

[13] R.H. Wagoner, J. V Laukonis, Metall. Trans. A 14 (1983) 1487-1495.

[14] R.H. Wagoner, Metall. Trans. A 13 (1982) 1491-1500.

[15] C. Zhou, Z. Chen, J.W. Lee, M.G. Lee, R.H. Wagoner, Int. J. Plast. 75 (2015) $121-140$. 
[16] Z. Chen, U. Gandhi, J. Lee, R.H. Wagoner, J. Mater. Process. Technol. 227 (2016) 227-243.

[17] Z. Chen, H.J. Bong, D. Li, R.H. Wagoner, Int. J. Plast. 83 (2016) 178-201.

[18] L. Sun, R.H. Wagoner, Int. J. Plast. 27 (2011) 1126-1144.

[19] J. da Costa Teixeira, L. Bourgeois, C.W. Sinclair, C.R. Hutchinson, Acta Mater. 57 (2009) 6075-6089.

[20] Y. Chen, M. Weyland, C.R. Hutchinson, Acta Mater. 61 (2013) 5877-5894.

[21] W. Prager, J. Appl. Mech. 23 (1956) 493-496.

[22] H. ZIEGLER, Q. Appl. Math. 17 (1959) 55-65.

[23] E.H. Edwards, J. Washburn, J. Met. 6 (1955) 1239-1242.

[24] M.Z. Wang, S. Lin, C.H. Li, Z.G. Wang, J. Xiao, Scr. Mater. 35 (1996) 1183-1188.

[25] B. Johannesson, S.L. Ogin, M.K. Surappa, P. Tsakiropoulos, S. Brynjolfsson, I.Ö. Thorbjornsson, Scr. Mater. 45 (2001) 993-1000.

[26] A.P. Reynolds, J.S. Lyons, Metall. Mater. Trans. A 28 (1997) 1205-1211.

[27] L.M. Brown, W.M. Stobbs, Philos. Mag. 23 (1971) 1185-1199.

[28] M.T. Ma, B.Z. Sun, Y. Tomota, ISIJ Int. 29 (1989) 74-77.

[29] E.A.J. Starke, Aluminum Alloys - Contemporary Research and Aplications, in: A. K., Academic Press, San Diego, CA, 1989.

[30] E. Orowan, (1948) 451-453.

[31] E. Orowan, in:, Intern. Stress. Fatigue Met., Detroit, 1958.

[32] J.D. Eshelby, Proc. R. Soc. London. Ser. A. Math. Phys. Sci. 241 (1957) 376 LP-396.

[33] W.F. Hosford, R.H. Zeisloft, Metall. Trans. 3 (1972) 113-121. 
[34] P. van Liempt, J. Sietsma, Mater. Sci. Eng. A 662 (2016) 80-87.

[35] D.L. McDowell, Mater. Sci. Eng. R Reports 62 (2008) 67-123.

[36] S. Tiwari, G.J. Tucker, D.L. McDowell, Philos. Mag. 93 (2013) 478-498.

[37] M. Kato, T. Fujii, S. Onaka, Mater. Trans. 49 (2008) 1278-1283.

[38] E.F. Rauch, J.-H. Schmitt, Mater. Sci. Eng. A 113 (1989) 441-448.

[39] T. Hasegawa, T. Yakou, U.F. Kocks, Mater. Sci. Eng. 81 (1986) 189-199.

[40] E. V Nesterova, B. Bacroix, C. Teodosiu, Mater. Sci. Eng. A 309-310 (2001) 495-499.

[41] J.H. Schmitt, E. Aernoudt, B. Baudelet, Mater. Sci. Eng. 75 (1985) 13-20.

[42] E.F. Rauch, Solid State Phenom. 23-24 (1992) 317-333.

[43] U.F. Kocks, T. Hasegawa, R.O. Scattergood, Scr. Metall. 14 (1980) 449-454.

[44] H. Mughrabi, Acta Metall. 31 (1983) 1367-1379.

[45] D. V Wilson, P.S. Bate, Acta Metall. Mater. 42 (1994) 1099-1111.

[46] Y. Strauven, E. Aernoudt, Acta Metall. 35 (1987) 1029-1036.

[47] J.A. Benito, J. Jorba, J.M. Manero, A. Roca, Metall. Mater. Trans. A 36 (2005) $3317-3324$.

[48] Z. Hu, E.F. Rauch, C. Teodosiu, Int. J. Plast. 8 (1992) 839-856.

[49] B. Peeters, M. Seefeldt, C. Teodosiu, S.R. Kalidindi, P. Van Houtte, E. Aernoudt, Acta Mater. 49 (2001) 1607-1619.

[50] B. Peeters, B. Bacroix, C. Teodosiu, P. Van Houtte, E. Aernoudt, Acta Mater. 49 (2001) 1621-1632.

[51] S. Bouvier, J.L. Alves, M.C. Oliveira, L.F. Menezes, Comput. Mater. Sci. 32 (2005) $301-315$. 
[52] A. Simar, Y. Bréchet, B. de Meester, A. Denquin, T. Pardoen, Acta Mater. 55 (2007) $6133-6143$.

[53] G. Fribourg, Y. Bréchet, A. Deschamps, A. Simar, Acta Mater. 59 (2011) $3621-3635$.

[54] W.Z. Han, A. Vinogradov, C.R. Hutchinson, Acta Mater. 59 (2011) 3720-3736.

[55] D. Bardel, M. Perez, D. Nelias, S. Dancette, P. Chaudet, V. Massardier, Acta Mater. 83 (2015) 256-268.

[56] G. Vincze, E.F. Rauch, J.J. Gracio, F. Barlat, A.B. Lopes, Acta Mater. 53 (2005) 1005-1013.

[57] F. Barlat, J. Liu, Mater. Sci. Eng. A 257 (1998) 47-61.

[58] P. Bate, W.T. Roberts, D.V. Wilson, Acta Metall. 29 (1981) 1797-1814.

[59] T. Dorin, F. De Geuser, W. Lefebvre, C. Sigli, A. Deschamps, Mater. Sci. Eng. A 605 (2014) 119-126.

[60] A. Deschamps, B. Decreus, F. De Geuser, T. Dorin, M. Weyland, Acta Mater. 61 (2013) 4010-4021.

[61] H. Proudhon, W.J. Poole, X. Wang, Y. Bréchet, Philos. Mag. 88 (2008) 621-640.

[62] R.K. Boger, R.H. Wagoner, F. Barlat, W. Gan, Prep. (2016).

[63] R.K. Boger, Influence of Hardening Precipitates on the Deformation of Aluminum Alloys, Ph. D Thesis, The Ohio State University, 2005.

[64] A. Deschamps, D. Dumont, Y. Brechet, C. Sigli, B. Dubost, in:, Proceeding James T. Stanley Honor. Symp. Alum. Alloy., Indianapolis, 2001.

[65] E. Hornbogen, A.. Mukhopadhyay, E.. Starke, Scr. Metall. Mater. 27 (1992) 733-738. 
[66] E. Hornbogen, A.K. Mukhopadhyay, E.A. Starke, Z Met. 83 (1992) 577-584.

[67] V. Radmilovic, A.J. Tolley, U. Dahmen, J.W. Morris, D. Mitlin, Metall. Mater. Trans. A 34 (2003) 543-551.

[68] E. Hornbogen, K.-H. Zum Gahr, Metallography 8 (1975) 181-202.

[69] F. Barlat, Materials Science Division, Alcoa Technical Center, Pittsburg, PA, 2005.

[70] J.L. Murray, A.J. McAlister, Bull. Alloy Phase Diagrams 5 (1984) 74.

[71] S.-I. Fujikawa, Y.-I. Izeki, Metall. Trans. A 24 (1993) 277-282.

[72] H.-M. Kagaya, K. Imazawa, M. Sato, T. Soma, Phys. B Condens. Matter 245 (1998) 252-255.

[73] S.M. Allen, Philos. Mag. A 43 (1981) 325-335.

[74] P.M. Kelly, A. Jostsons, R.G. Blake, J.G. Napier, Phys. Status Solidi 31 (1975) $771-780$.

[75] J.W. Martin, Precipitation Hardening, 2nd edn, Butterworth-Heinemann, Oxford, 1998.

[76] ASTM E8 / E8M-15a, Standard Test Methods for Tenstion Testing of Metallic Materials, ASTM International, West Conshohocken, PA, 2015.

[77] R.K. Boger, R.H. Wagoner, F. Barlat, M.G. Lee, K. Chung, Int. J. Plast. 21 (2005) 2319-2343.

[78] W. Gan, K. Okamoto, S. Hirano, K. Chung, C. Kim, R.H. Wagoner, J. Eng. Mater. Technol. 130 (2008) 31007.

[79] V. Balakrishnan, Measurement of in-Plane Bauschinger Effect in Metal Sheet, MS Thesis, The Ohio State University, 1999.

[80] K. Piao, J.K.K. Lee, J.H.H. Kim, H.Y.Y. Kim, K. Chung, F. Barlat, R.H. Wagoner, 
Int. J. Plast. 38 (2012) 27-46.

[81] K. Piao, K. Chung, M.-G. Lee, R.H. Wagoner, Metall. Mater. Trans. A 43 (2012) $3300-3313$.

[82] Laser Extensometer, Model LE-01, PA 15642, 2005.

[83] A. Abel, Mater. Forum 10 (1987) 11-26.

[84] A. Abel, H. Muir, Philos. Mag. 26 (1972) 489-504.

[85] X.Y. Lou, Evolution of Hardening in AZ31B Mg Sheet, The Ohio State University, 2005.

[86] N.L. Peterson, S.J. Rothman, Phys. Rev. B 1 (1970) 3264-3273.

[87] D. Simonovic, M.H.F. Sluiter, Phys. Rev. B 79 (2009) 54304.

[88] T. Zumkley, H. Mehrer, Zeitschrift Für Met. 89 (1998) 454-463.

[89] N. Hansen, Acta Metall. 25 (1977) 863-869.

[90] L.M. Cheng, W.J. Poole, J.D. Embury, D.J. Lloyd, Metall. Mater. Trans. A 34 (2003) 2473-2481.

[91] U.F. Kocks, H. Mecking, Prog. Mater. Sci. 48 (2003) 171-273.

[92] P. Haasen, in:, n.d.

[93] M.Z. Butt, P. Feltham, J. Mater. Sci. 28 (1993) 2557-2576.

[94] U.F. Kocks, Metall. Trans. A 16 (1985) 2109-2129.

[95] M.G. Stout, A.D. Rollett, Metall. Trans. A 21 (1990) 3201-3213.

[96] W. Gan, P. Zhang, R.H. Wagoner, G.S. Daehn, Metall. Mater. Trans. A 37 (2006) 2097-2106.

[97] X. Hu, C. Wei, H. Margolin, S. Nourbakhsh, Scr. Metall. Mater. 27 (1992) 865-870. 
[98] N. Anjabin, A. Karimi Taheri, Mater. Des. 31 (2010) 433-437.

[99] T.H. Courtney, Mechanical Behavior of Materials: Second Edition, Waveland Press, 2005.

[100] S.R. (Surya R. Kalidindi, Polycrystal Plasticity: Constitutive Modeling and Deformation Processing, Massachusetts Institute of Technology, 1992.

[101] P.R. Dawson, Int. J. Solids Struct. 37 (2000) 115-130.

[102] D. Raabe, P. Klose, B. Engl, K.-P. Imlau, F. Friedel, F. Roters, Adv. Eng. Mater. 4 (2002) 169-180.

[103] A. Ma, F. Roters, D. Raabe, Acta Mater. 54 (2006) 2169-2179.

[104] D. Raabe, D. Ma, F. Roters, Acta Mater. 55 (2007) 4567-4583.

[105] H. Lim, M.G. Lee, J.H. Kim, B.L. Adams, R.H. Wagoner, Int. J. Plast. 27 (2011) $1328-1354$.

[106] N.A.N. Fleck, G.M.G. Muller, M.F. Ashby, J.W. Hutchinson, Acta Metall. Mater. 42 (1994) 475-487.

[107] W. Nix, H. Gao, J. Mech. Phys. Solids (1998).

[108] P. Gudmundson, J. Mech. Phys. Solids (2004).

[109] M.G. Lee, H. Lim, B.L. Adams, J.P. Hirth, R.H. Wagoner, Int. J. Plast. 26 (2010) 925-938.

[110] H. Lim, S. Subedi, D.T. Fullwood, B.L. Adams, R.H. Wagoner, Mater. Trans. 55 (2014) 35-38.

[111] H.J. Bong, H. Lim, M.-G. Lee, D. Fullwood, E. Homer, R.H. Wagoner, Mater. Sci. Eng. A (2016) In preparation.

[112] J.R. Rice, J. Mech. Phys. Solids 19 (1971) 433-455. 
[113] D. Peirce, R.J. Asaro, A. Needleman, Acta Metall. 30 (1982) 1087-1119.

[114] R.J. Asaro, Advances in Applied Mechanics Volume 23, Elsevier, 1983.

[115] J. Gubicza, N.Q. Chinh, J.L. Lábár, S. Dobatkin, Z. Hegedűs, T.G. Langdon, J. Alloys Compd. 483 (2009) 271-274.

[116] G. Schoeck, R. Frydman, Phys. Status Solidi (1972).

[117] U.F. Kocks, J. Eng. Mater. Technol. Trans. ASME 98 Ser H (1976) 76-85.

[118] E. Orowan, Proc. Phys. Soc. 52 (1940) 8-22.

[119] J.P. Hirth, J. Lothe, Theory of Dislocations, Krieger Publishing Company, 1982.

[120] Z. Shen, R.H. Wagoner, W.A.T. Clark, Scr. Metall. 20 (1986) 921-926.

[121] W.. Hosford, R.. Fleischer, W.. Backofen, Acta Metall. 8 (1960) 187-199.

[122] M. Abbadi, P. Hähner, A. Zeghloul, Mater. Sci. Eng. A 337 (2002) 194-201.

[123] S.L. Mannan, Bull. Mater. Sci. 16 (1993) 561-582. 